"Glucose-nucleobase pseudo base pairs as a new biomolecular interaction in a DNA context" Vengut-Climent, E., Gómez-Pinto, I., Lucas, R., Peñalver, P., Aviñó, A., Fonseca-Guerra, C, Bickelhaupt, F.M.., Eritja, R., González, C., Morales, J.C. Angew. Chem. Int. Ed., 55(30), 8643-8647, (2016). doi: 10.1002/anie201603510

\title{
Glucose-Nucleobase Pseudo Base Pairs: Biomolecular Interactions within DNA
}

\author{
Empar Vengut-Climent, ${ }^{a}$ Irene Gómez-Pinto, ${ }^{\mathrm{b}}$ Ricardo Lucas, ${ }^{\mathrm{a}, \mathrm{c}}$ Pablo \\ Peñalver, ${ }^{a, c}$ Anna Aviñó, ${ }^{d}$ Célia Fonseca Guerra, ${ }^{e}$ F. Matthias \\ Bickelhaupt, ${ }^{\mathrm{e}, \mathrm{f}}$ Ramón Eritja, ${ }^{\mathrm{d}}$ Carlos González, ${ }^{\mathrm{b}}$ and Juan C. \\ Morales*a,c
}

${ }^{a}$ Department of Bioorganic Chemistry, Instituto de Investigaciones Químicas CSIC-Universidad de Sevilla, Américo Vespucio 49, 41092 Sevilla (Spain) E-mail: jcmorales@ipb.csic.es

bInstituto de Química Física "Rocasolano", CSIC 28006 Madrid (Spain)

"Department of Biochemistry and Molecular Pharmacology, Instituto de Parasitología y Biomedicina, CSIC, Parque Tecnológico, Ciencias de la Salud 18016 Armilla, Granada (Spain)

dinstituto de Química Avanzada de Cataluña (IQAC), CSIC, CIBER-BBN Networking Centre on Bioengineering, Biomaterials and Nanomedicine, 08034 Barcelona (Spain),

eDepartment of Theoretical Chemistry and Amsterdam Center for Multiscale Modeling, Vrije Universiteit Amsterdam 1081 HV Amsterdam (The Netherlands),

fInstitute of Molecules and Materials (IMM), Radboud University, 6525 AJ Nijmegen (The Netherlands).

Abstract: Noncovalent forces rule the interactions between biomolecules. Inspired by a biomolecular interaction found in aminoglycoside-RNA recognition, glucose-nucleobase pairs have been examined. Deoxyoligonucleotides with a 6-deoxyglucose insertion are able to hybridize with their complementary strand, thus exhibiting a preference for purine nucleobases. Although the resulting double helices are less stable than natural ones, they present only minor local distortions. 6-Deoxyglucose stays fully integrated in the double helix and its $\mathrm{OH}$ groups form two hydrogen bonds with the opposing guanine. This 6-deoxyglucose-guanine pair closely resembles a purine-pyrimidine geometry. Quantum chemical calculations indicate that glucose-purine pairs are as stable as a natural T-A pair.

Noncovalent forces govern interactions among biomolecules, drugtarget molecular recognition and assembly processes. Hydrogen bonds, $\pi-\pi$ stacking, van der Waals forces, electrostatic forces, and hydrophobic 
interactions are observed in RNA recognition by drugs such as macrolides, tetracyclines,[1] and new designed RNA binders.[2] Aminoglycoside antibiotics are a well-known family of RNA binders which target the 16S rRNA in the small ribosomal subunit. Apart from electrostatic forces and hydrogen bonds, solution and X-ray structures of aminoglycosides with rRNA show a singular biomolecular interaction, a monosaccharidenucleobase stacking interaction [e.g., 2'-amino-2'-deoxyglucose (ring I) of paromomycin stacks over guanine 1491; Figure 1].[3]

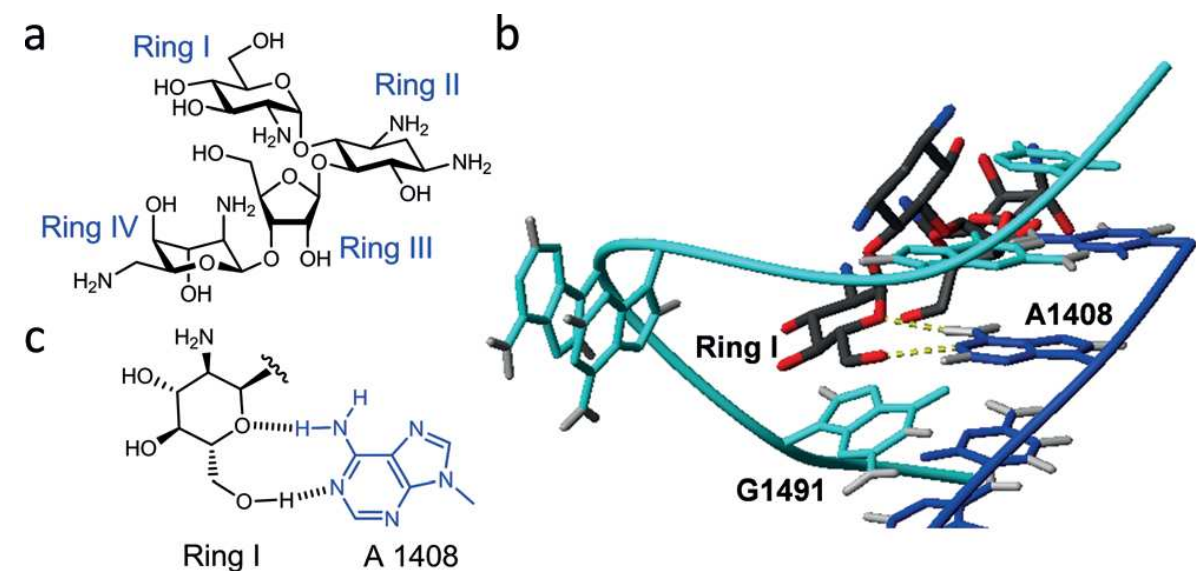

Figure 1. a) Structure of paromomycin. b) Detail of the solution structure of paromomycin binding a $16 \mathrm{~S}$ RNA model sequence. c) Drawing of the glycosideadenine 4108 pseudo base pair.

Recently, we have studied sugar-DNA stacking interactions using a self-complementary CGCGCG sequence with the carbohydrates directly linked to the 5'-end of DNA. We observed stacking of mono- and disaccharides on top of the terminal DNA base pairs, which stabilized the duplex between -0.5 to $-1.8 \mathrm{kcalmol}^{-1}$.[4] Stacking of sugars on top of the guanine tetrad of a DNA G-quadruplex was also characterized.[4b]

A second singular biomolecular interaction found when paramomycin binds rRNA is a glycoside-adenine pseudo base pair (Figure 1c).[3] Two hydrogen bonds are formed between ring I of paromomycin and A1408. Inspired by this interaction we decided to study the possible formation of glucosenucleobase pairs. Since monosaccharides can stack on DNA bases and possess $\mathrm{OH}$ groups capable of making hydrogen bonds at the edge of their coinlike structure, like natural bases (Figure 2a), we placed a potential glucose-nucleobase pair inside a DNA double helix to investigate its properties (Figure $2 \mathrm{~b}$ ). This model system also allows us to study possible sugar-sugar pairs. The only non-aromatic nucleobases reported previously are Leumann's phenyl cyclohexyl interstrand base[5] and Kashida_s isopropylcyclohexane and methylcyclohexane DNA base insulators.[6] 


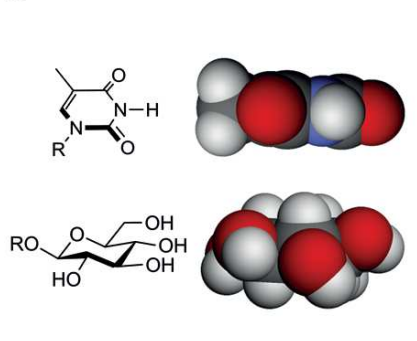

C
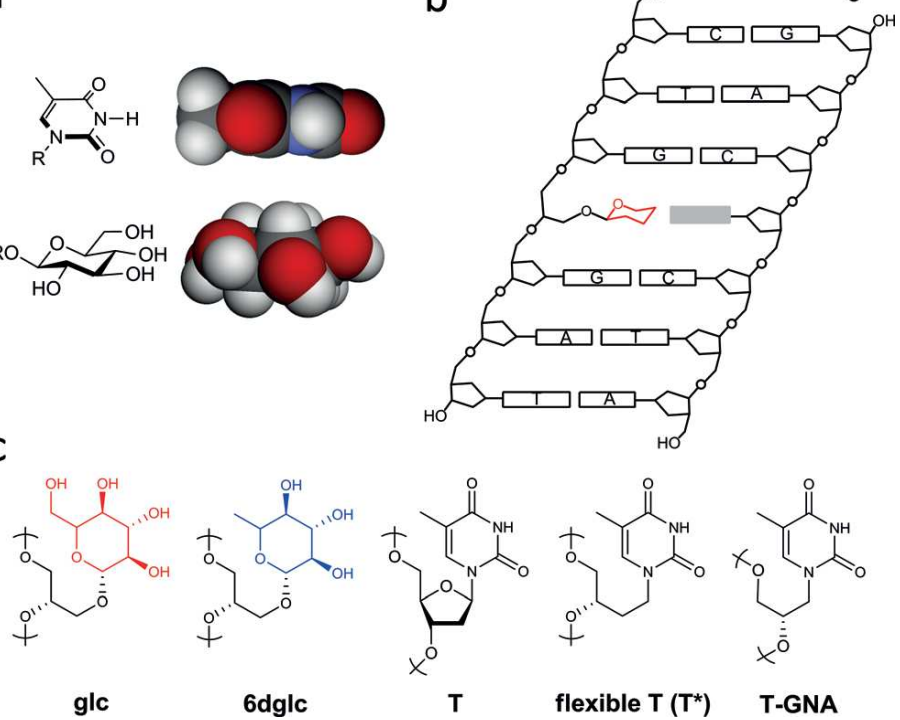

flexible $T\left(T^{*}\right) \quad$ T-GNA

Figure 2. Description of the carbohydrate derivatives under study. a) CPK models of thymine and glucose. b) Schematic drawing of a DNA double helix containing a glycoside-nucleobase pair. c) Structures of the two sugar units prepared, (S)-2,3dihydroxypropyl glucose (glc) and (S)-2,3-dihydroxypropyl 6-deoxyglucose (6dglc), thymidine $(T),(S)-3,4$-dihydroxybutyl thymine or flexible $T\left(T^{*}\right)$, and glycol $T(T-$ GNA).

Glucose (glc) was linked to the phosphodiester backbone of DNA through a flexible glycerol linker through its anomeric position. Thus, we prepared the DMT-glucose phosphoramidite derivative 9 (Scheme 1) for introduction at the required position of the oligonucleotide, similar to a standard natural DNA base phosphoramidite. To modulate the high polarity of glucose, we also prepared the $6 \mathrm{dglc}$ amidite 10. A flexible $T\left(T^{*}\right)$ was synthesized for comparison of glc with a pyrimidine base in the same environment (Figure 2c; see Figure S1 in the Supporting Information).

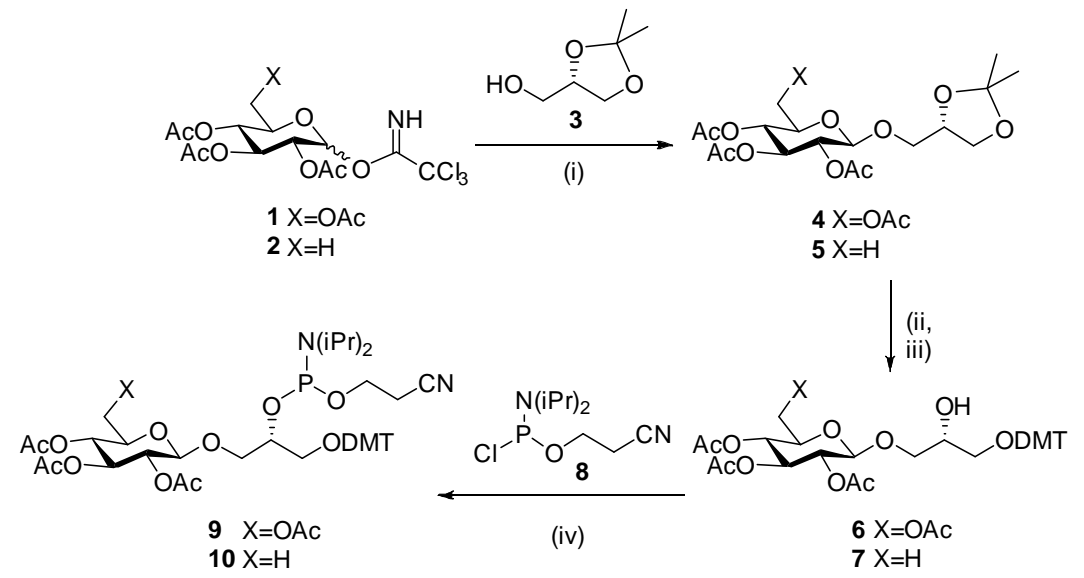

Scheme 1. Synthesis of the carbohydrate DMT phosphoramidites. Glycosyl trichloroacetimidate donors were used as starting material. Reagents and conditions: (i) TMSOTf, $\mathrm{CH}_{2} \mathrm{Cl}_{2}$ for glc; $\mathrm{BF}_{3} \cdot \mathrm{OEt}_{2}, \mathrm{CH}_{2} \mathrm{Cl}_{2}$ for 6 dglc; (ii) $\mathrm{AcOH}-\mathrm{H}_{2} \mathrm{O}, 80^{\circ} \mathrm{C}$; (iii) DMTCl, DMAP, 
$\mathrm{CH}_{2} \mathrm{Cl}_{2} ;$ (iv) DIPEA, $\mathrm{CH}_{2} \mathrm{Cl}_{2}$. DIPEA=diisopropylethylamine, DMAP=4-(N,Ndimethylamino) pyridine, $\mathrm{DMT}=4,4^{\prime}$-dimethoxytrityl, $\mathrm{Tf}=$ =trifluoromethanesulfonyl, $\mathrm{TMS}=$ trimethylsilyl.

Table 1. Melting temperature (Tm) for DNA duplexes containing $T^{*}$, glc and 6dglc and GNA duplexes containing 6dglc

\begin{tabular}{|c|c|c|c|c|c|}
\hline \multicolumn{2}{|c|}{ DNA duplexes } & \multicolumn{4}{|c|}{$5^{\prime}-d(G A T G A C X G C T A G)^{[a, b]}$} \\
\hline \multicolumn{6}{|c|}{$3^{\prime}-d(C T A C T G Y C G A T C)$} \\
\hline$X-Y$ & $T_{m}\left({ }^{\circ} \mathrm{C}\right)$ & $X-Y$ & $T_{m}\left({ }^{\circ} \mathrm{C}\right)$ & $X-Y$ & $T_{m}\left({ }^{\circ} \mathrm{C}\right)$ \\
\hline$T *-A$ & 37.7 & alc-A & 30.6 & 6dalc-A & 33.2 \\
\hline $\mathrm{T} *-\mathrm{T}$ & 32.8 & glc-T & 28.5 & $6 d g l c-T$ & 29.9 \\
\hline $\mathrm{T}^{*}-\mathrm{C}$ & 32.0 & glc-C & 28.7 & 6dglc-C & 29.9 \\
\hline $\mathrm{T}^{*}-\mathrm{G}$ & 35.6 & glc-G & 31.3 & $6 \mathrm{dglc}-\mathrm{G}$ & 32.7 \\
\hline T*_T* & 31.1 & alc- & 29.6 & 6dalc-6dalc & 32.0 \\
\hline \multicolumn{2}{|c|}{ GNA dunlexes } & \multicolumn{3}{|c|}{$3^{\prime}-T A A A A T T A \mathbf{X} A T T A T T A A^{[b, c]}$} & \\
\hline$X-Y$ & $T_{m}$ & \multicolumn{3}{|c|}{ 2'-АTाTAAATYTAATAATT } & $T_{m}\left({ }^{\circ} \mathrm{C}\right)$ \\
\hline$T-A$ & 57.3 & & & A-6dalc & 45.4 \\
\hline$T-T$ & 48.0 & & & T-6dalc & 37.4 \\
\hline & & & & 6dalc-T & 38.7 \\
\hline & & & & 6dalc-6dalc & 43.0 \\
\hline
\end{tabular}

[a] The natural DNA duplex containing $X-Y=T-A$ results in a $T_{m}$ value of $47.98 \mathrm{C}$. [b] Conditions for DNA duplexes: $10 \mathrm{~mm} \mathrm{NaH} \mathrm{PO}_{4}, 150 \mathrm{mM} \mathrm{NaCl}, \mathrm{pH}$ 7.0. For GNA duplexes: $10 \mathrm{mM} \mathrm{NaH}{ }_{2} \mathrm{PO}_{4}, 200 \mathrm{mM} \mathrm{NaCl}, \mathrm{pH} 7.0$. Estimated errors are: $\pm 0.4{ }^{\circ} \mathrm{C}$ (in DNA, except for $6 \mathrm{dglc}-6 \mathrm{dglc}: \pm 1.0^{\circ} \mathrm{C}$ ) and $\pm 1.0^{\circ} \mathrm{C}(\mathrm{GNA})$. Average value of three experiments measured at $1.2 \mu \mathrm{M}$ conc (DNA) and $0.7 \mu \mathrm{M}$ conc (GNA). [c] GNA monomers in italics.

We then measured thermal denaturation of DNA duplexes containing our modifications. Firstly, the introduction of a flexible linker, such as in $T^{*}$, with respect to a natural $\mathrm{T}$ leads to a $10.2-15.9{ }^{\circ} \mathrm{C}$ decrease in stability (Table 1). The decrease in stability of DNA containing single or multiple acyclic nucleosides has been previously reported.[7] Secondly, the DNA duplexes with glc and $6 \mathrm{dglc}$ opposite to the four natural nucleosides were destabilized in comparison to the duplexes with T-A and $\mathrm{T}^{*}-\mathrm{A}$. Considering the penalty of the flexible linker, destabilization of glc pairs compared to $\mathrm{T}^{*}$ A ranges from 6.4 to $9.2^{\circ} \mathrm{C}$. Interestingly, the presence of the more apolar 6 dglc improved the DNA stability, when compared to glc, by $1.2-3.9{ }^{\circ} \mathrm{C}$. Still, the more stable pair, 6dglc-A, led to a DNA duplex which is $4.5^{\circ} \mathrm{C}$ less stable than that with $\mathrm{T}^{*}-\mathrm{A}$, and $15.7^{\circ} \mathrm{C}$ less stable than that with $\mathrm{T}-\mathrm{A}$. The larger volume of the pyranose ring, in comparison with an aromatic ring, may distort the nearby DNA base pairs and provoke this decrease in DNA stability. Thirdly, we observed certain selectivity for glc-nucleobase and 6dglc-nucleobase pairs. Sugar-purine pairs were more stable than 
sugarpyrimidine pairs (from 1.9 to $3.3{ }^{\circ} \mathrm{C}$ ). This difference may be a consequence of direct hydrogen bonding between the $\mathrm{OH}$ groups of glc and $6 \mathrm{dglc}$ and the donor and acceptor groups in A and G. Alternatively, purines may be preferred in front of glucose in the sequence just for the better stacking as observed in abasic sites,[8] but the NMR spectroscopy and theoretical calculations shown below indicate that hydrogen bonding between glc and $6 \mathrm{dglc}$ with $A$ and $G$ is possible. Fourthly, the stability of DNA duplexes containing sugar-sugar pairs ranges from 29.6 to $32.3{ }^{\circ} \mathrm{C}$. Unexpectedly, pairs containing two flexible spacers and two bulky pyranose rings do not show further DNA destabilization in comparison to sugarnatural nucleobase pairs. For example, a 6 dglc-6dglc pair is as stable as a 6 dglc purine pair.

We also incorporated glc and 6dglc into a glycerol nucleic acid (GNA) double helix. GNA strands have an acyclic backbone of propylene glycol nucleosides which incorporate the natural nucleobases in the strand and are connected by phosphodiester bonds (Figure 2c).[9] In this context, both natural bases and our sugar modifications are linked through flexible linkers to the skeleton and no energetic penalty is expected. The Tm values measured showed that the GNA duplex with an A-6dglc pair was $11.98 \mathrm{C}$ less stable than with T-A (Table 1 ) and only $2.68 \mathrm{C}$ less stable than with a mismatched T-T. It is quite notable that the selectivity between T-A and T-T pairs $(9.38 \mathrm{C})$ is similar to that between $6 \mathrm{dglc}-\mathrm{A}$ and $6 \mathrm{dglc}-\mathrm{T}$ pairs $(6.7-8.0$ $\left.{ }^{\circ} \mathrm{C}\right)$. Lastly, a $6 \mathrm{dglc}-6 \mathrm{dg}$ lc pair results in a GNA duplex stability of $43^{\circ} \mathrm{C}$, thus pointing to the formation of hydrogen bonds between the $\mathrm{OH}$ groups of each 6dglc unit.

We next determined the three-dimensional high-resolution structure of DNA duplexes containing a $6 \mathrm{dglc}-\mathrm{G}$ and a $6 \mathrm{dglc}-T$ pair using restrained molecular dynamics methods based on experimental NMR distance constraints (Figure 3). The exchangeable proton region of the NMR spectra exhibited 11 imino proton signals between $\mathrm{d}=12.5$ and $14.5 \mathrm{ppm}$, thus indicating the formation of a duplex structure with Watson-Crick base pairs (see Figure S4). Additional imino proton signals are observed in the $\mathrm{d}=10-$ $11 \mathrm{ppm}$ region corresponding to the nucleotide located opposite to the carbohydrate. Full proton assignment of the DNA and sugar units was performed with only a few exceptions (see Tables S1 and S2). DNA chemical-shift differences between the conjugates and the natural DNA control duplexes are limited to the surroundings of the carbohydrate moieties, thus indicating the overall duplex structure is not distorted (see Figure S5).

The 3D structures obtained are B-form helices without global distortions (Figure 3; see Figures S7 and S8). The carbohydrates and the nucleobases ( $\mathrm{G}$ or $\mathrm{T}$ ) located in the opposite position remain intercalated between the surrounding base-pairs maintaining extensive contacts at both sides. As a consequence, a large number of NOE crosspeaks (see Table S3) between the linker and the carbohydrate protons with the DNA are observed. In all cases the double helices are slightly unwound and the rise between flanking residues is increased, as usually occurs in intercalation complexes. 

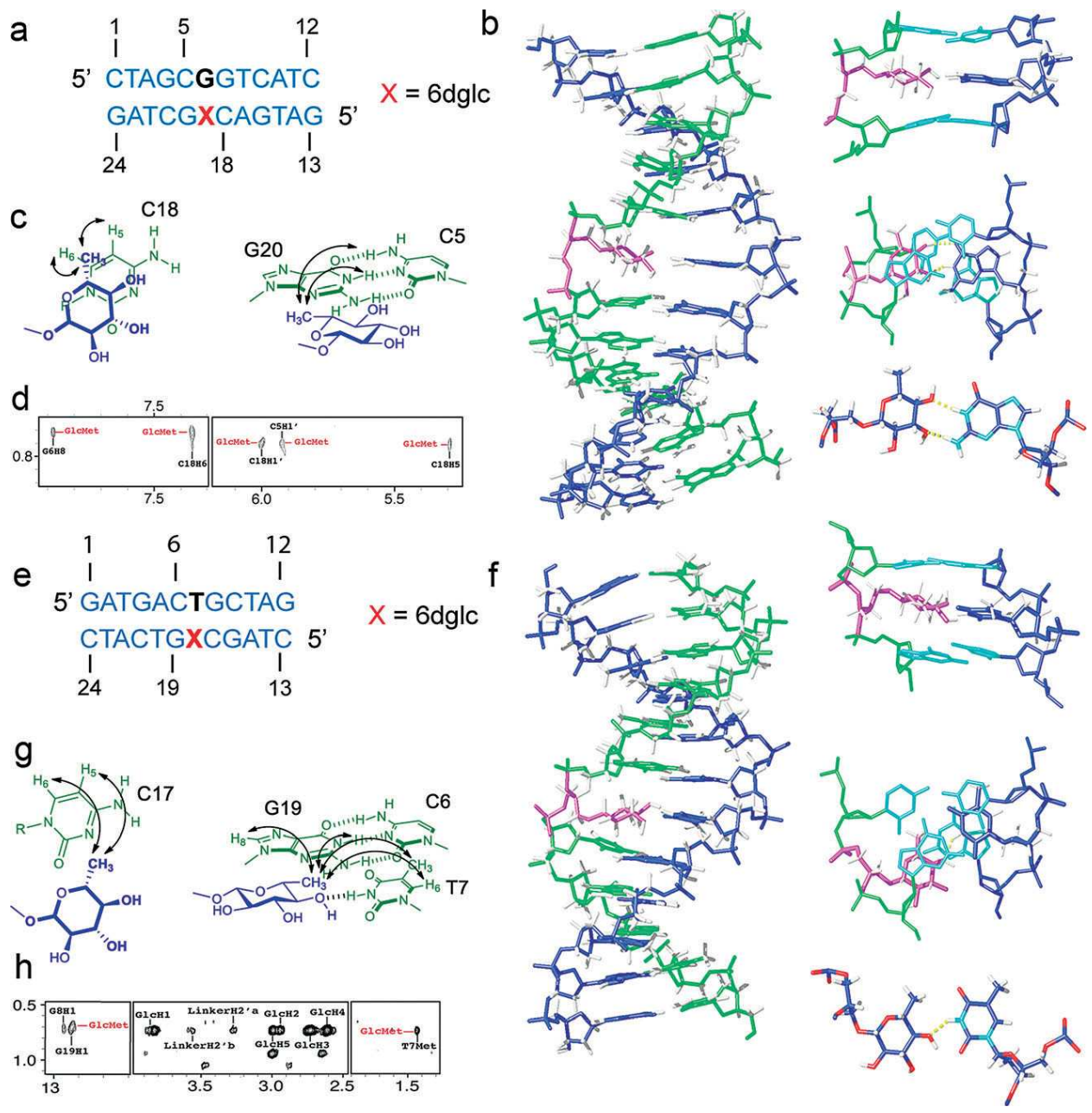

Figure 3. Solution structure of the helix $6 \mathrm{dglc}-\mathrm{G}$ and helix $6 \mathrm{dglc}-\mathrm{T}$. a) Sequences of the helix 6 dglc-G. b) Refined solution structure of the helix $6 \mathrm{dglc}-\mathrm{G}$ and zoom views of the $6 \mathrm{dglc}-\mathrm{G}$ pair. c) NOE interactions between $6 \mathrm{dglc}$ and its $5^{\prime}$-neighboring base C18 and its 3'- neighboring base pair G20-C5. d) NOESY trace showing key NOEs with the methyl group of $6 \mathrm{dglc}$. e) Sequences of helix 6dglc-T. f) Refined solution structure of helix $6 \mathrm{dglc}-T$ and zoom views of the $6 \mathrm{dglc}-T$ pair. g) NOE interactions between 6dglc and its 5'-neighbouring base C17, and with its 3'-neighboring base pair G19-C6 and opposite base T7. h) NOESY trace showing key NOEs with the methyl group of 6dglc. Color code: sugar and spacer (purple), nucleobases (green and blue) and surrounding natural base pairs (light blue).

The structures of helix $6 \mathrm{dglc}-\mathrm{G}$ and helix $6 \mathrm{dglc}-\mathrm{T}$ are deposited in the PDB (2N9Fand $2 \mathrm{N9H}$, respectively). $6 \mathrm{dglc}$ is well located opposite to both guanine (Figure $3 \mathrm{~b}$ ) and thymine (Figure $3 \mathrm{f}$ ), thus orienting its alpha face towards its 3'-neighboring nucleobase and its methyl group towards the major groove. In the helix 6dglc-G the sugar-nucleobase pair forms two hydrogen bonds. In six of the ten resulting structures, these hydrogen bonds are $\mathrm{H} 1 \mathrm{G}-\mathrm{O} 4 \mathrm{6dglc}$ and $\mathrm{HN} 2 \mathrm{G}-\mathrm{O} 3$ 6dglc (Figure 3b). The other structures show different conformers, including the pattern obtained in the quantum calculations (see below). All orientations are experimentally 
supported by a number of intra- and interstrand NOEs, like those between the methyl group of $6 \mathrm{dglc}$ with $\mathrm{H} 5$ and $\mathrm{H} 6 \mathrm{C} 18$, with amino protons of $\mathrm{C} 5$, and with $\mathrm{H} 1 \mathrm{G} 20$ (Figure 3c). In the helix 6dglc-T the monosaccharide forms only one hydrogen bond with the opposing $\mathrm{T}$. In most structures ( 7 out of 10 ), this hydrogen bond is $\mathrm{H} 3 \mathrm{~T}-\mathrm{O} 4$ 6dglc (Figure $3 \mathrm{f}$ ), and in the other cases the hydrogen bond is $\mathrm{HO} 46 \mathrm{dglc}-\mathrm{O} 2 \mathrm{~T}$. The orientation is supported by NOEs, for example, between the methyl group of 6dglc with methyl and $\mathrm{H} 6 \mathrm{T7}$; with $\mathrm{H} 1$ and $\mathrm{H} 8 \mathrm{G} 19$; and with $\mathrm{H} 5$ and $\mathrm{H} 6 \mathrm{C} 17$ (Figure $3 \mathrm{~g}$ ). The formation of an extra hydrogen bond, when comparing $6 \mathrm{dglc}-\mathrm{G}$ and $6 \mathrm{dglc}-\mathrm{T}$, may contribute to the enhanced thermal stability and it may explain the observed selectivity for purines.

The pairing properties of glc and $6 \mathrm{dglc}$ in the gas phase and water were investigated quantum chemically by means of dispersion-corrected density functional theory (DFT) at the BLYP-D3(BJ)/TZ2P level of theory.[10] The binding energies (DE) of the Watson-Crick base pairs A-T and G-C agreed with those reported in literature (Table 2).[11] Sugarpurine pairs show greater hydrogen-bonding energy than any other combination for both for glc and $6 \mathrm{dglc}$. In fact, the G-6dglc pair shows greater energy of bonding than a natural A-T base pair, with two hydrogenbonds between the $\mathrm{OH} 3$ and $\mathrm{OH} 4$ of 6 dglc with the $\mathrm{NH} 1$ and $\mathrm{C}=\mathrm{O}$, respectively, of $\mathrm{G}$ (Figure 4 ).

Table 2. Hydrogen-bond energies (in $\mathrm{kcal} \cdot \mathrm{mol}^{-1}$ ) of sugar-base pairs in the gas-phase $\left(\Delta E_{\text {gas }}\right)$ and in aqueous solution $\left(\Delta E_{\text {water }}\right)$. ${ }^{\text {[a] }}$

\begin{tabular}{lrrrrr}
\hline $\mathrm{X}-\mathrm{Y}$ & $\square E_{\text {gas }}$ & $\Delta E_{\text {wat }} \mathrm{e}$ & $\mathrm{X}-\mathrm{Y}$ & $\Delta E_{\text {gas }}$ & $\Delta E_{\text {water }}$ \\
\hline $\mathrm{A}-\mathrm{T}$ & -18.5 & -9.4 & $\mathrm{G}-\mathrm{C}$ & -34.0 & -13.5 \\
\hline 6dglc-G & -23.8 & -10.5 & glc-G & -23.3 & -12.2 \\
6dglc-T & -9.1 & -5.0 & glc-T & -15.4 & -9.5 \\
6dglc-A & -16.7 & -10.5 & glc-A & -16.7 & -10.7 \\
6dglc-C & -12.9 & -6.7 & glc-C & -17.7 & -5.1 \\
6dglc- & -8.3 & -4.1 & glc-glc & -12.7 & -8.7 \\
6dglc & & & & & \\
6dglc-glc & -11.4 & -7.6 & & & \\
\hline
\end{tabular}

${ }^{[a]}$ Calculated at BLYP-D3(BJ)/TZ2P using COSMO to simulate aqueous solution. 
a

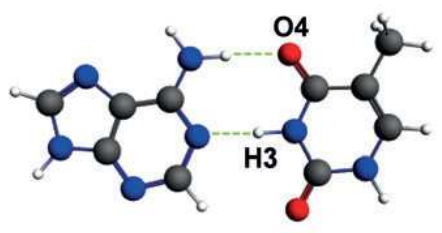

A-T

C

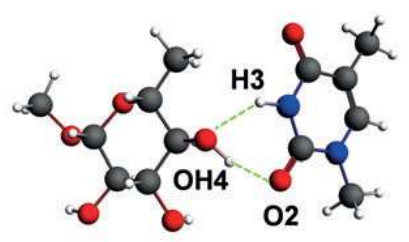

6dglc-T b

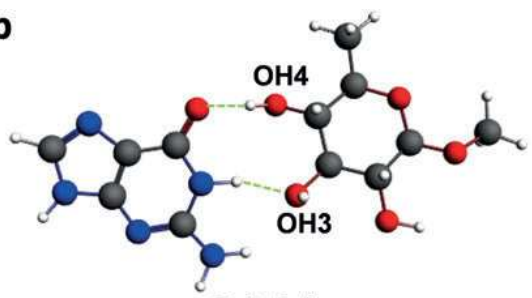

G-6dglc

d

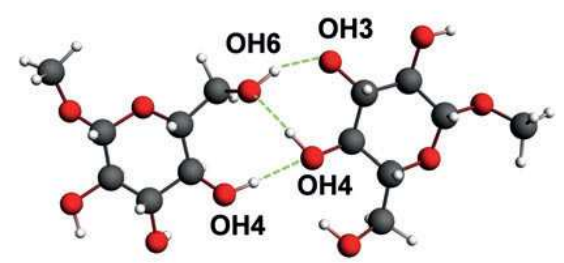

glc-glc

Figure 4. A-T, G-6dglc, 6dglc-T, and glc-glc pairs calculated at BLYPD3( $\mathrm{BJ}) / \mathrm{TZ2P}$ using COSMO to simulate aqueous solution. Hydrogen bonds are shown in green.

Two hydrogen bonds are also observed for A-6dglc, G-glc, and A-glc pairs (see Figure S9). Differences in the hydrogenbonding pattern in comparison to the NMR structures (such as in $6 \mathrm{dglc}-\mathrm{T}$ ) may be ascribed to factors such as base-base stacking or distance restrictions resulting from the DNA skeleton, and these are factors which are not included in these calculations.

Small differences between glc and 6 dglc were observed when pairing with purines. In fact, the hydrogen bond formed between the $\mathrm{OH} 4$ of glc and the $\mathrm{C}=\mathrm{O}$ of $\mathrm{G}$ is reinforced through a third hydrogen bond which is formed between the $\mathrm{OH} 6$ and $\mathrm{OH} 4$ (see Figure S9), thus yielding a better energy of bonding for glc-G than for $6 \mathrm{dglc}-\mathrm{G}$. Note that glc and 6dglc form significantly more stable pairs with pyrimidines than with purines. The only exception is the glc-T pair which has a bond energy of $-9.55 \mathrm{kcalmol}^{-1}$.

Interestingly, sugar-sugar base pairs show similar bond energies to that of sugar-pyrimidine base pairs. One, two, and three hydrogen bonds are formed in $6 \mathrm{dglc}-6 \mathrm{dglc}$, $6 \mathrm{dglc}-\mathrm{glc}$, and glc-glc, respectively, where the $\mathrm{OH}$ groups involved can act as either hydrogen-bond donors or acceptors (Figure 4d). In contrast to the experimental data found for DNA, the 6dglc6 dglc pair appears to be less stable than the glc-glc base pair. These differences may be ascribed to interactions with the surrounding bases,[11] which are not considered in the present calculations.

In conclusion, we have shown glycoside-nucleobase pairs form within a DNA double helix. They cause some destabilization of either a DNA or GNA duplex but they display selective pairing with purines versus pyrimidines. This selectivity can be explained by the formation of hydrogen bonds between either glc or $6 \mathrm{dglc}$ with the natural bases as shown by quantum chemical calculations and NMR studies. Moreover, 6dglc stacks within the 
interior of a duplex when paired with either $\mathrm{G}$ or $\mathrm{T}$ and does not stick out of the helix seeking better hydration. These $6 \mathrm{dglc}-\mathrm{G}$ and $6 \mathrm{dglc}-T$ pairs infer only minor distortion in the double-helix structure.

\section{Acknowledgments}

We thank the Ministerio de Economía y Competitividad (CTQ201115203-E, CTQ2012-35360, CTQ2014-52588-R, BFU2014-52864-R), the Netherlands Organization for Scientific Research (NWO-CW and NWO-EW), and the National Research School Combination-Catalysis (NRSC-C) for financial support. E.V.C. thanks Ministerio de Educación, Cultura y Deporte for a FPU fellowship and Cost Action CM1005 for a STSM grant.

Keywords: DNA; hydrogen bonds; NMR spectroscopy; noncovalent interactions; nucleobases

[1] A. Blond, E. Ennifar, C. Tisne, L. Micouin, ChemMedChem 2014, 9, 1982 $-1996$.

[2] a) R. Moumné, M. Catala, V. Larue, L. Micouin, C. Tisné, Biochimie 2012, 94, 1607 - 1619; b) S. P. Velagapudi, B. R. Vummidi, M. D. Disney, Curr. Opin. Chem. Biol. 2015, 24, 97- 103.

[3] Q. Vicens, E. Westhof, Biopolymers 2003, 70, 42 - 57.

[4] a) R. Lucas, I. Gómez-Pinto, A. Aviñó, J. J. Reina, R. Eritja, C. González, J. C. Morales, J. Am. Chem. Soc. 2011, 133, 1909 - 1916; b) I. GómezPinto, E. Vengut-Climent, R. Lucas, A. Aviñó, R. Eritja, C. González, J. C. Morales, Chem. Eur. J. 2013, 19, 1920 - 1927; c) R. Lucas, P. Peñalver, I. Gomez-Pinto, E. Vengut-Climent, L. Mtashobya, J. Cousin, O. S. Maldonado, V. Perez, V. Reynes, A. Aviñó, R. Eritja, C. González, B. Linclau, J. C. Morales, J. Org. Chem. 2014, 79, 2419 - 2429.

[5] M. Kaufmann, M. Gisler, C. J. Leumann, Angew. Chem. Int. Ed. 2009, 48, 3810 - 3813; Angew. Chem. 2009, 121, 3868 - 3871.

[6] H. Kashida, K. Sekiguchi, H. Asanuma, Chem. Eur. J. 2010, 16, 11554 11557.

[7] a) K. C. Schneider, S. A. Benner, J. Am. Chem. Soc. 1990, 112, 453 455; b) D. Zhou, I. M. Lagoja, J. Rozenski, R. Busson, A. Van Aerschot, P. Herdewijn, ChemBioChem 2005, 6, 2298 - 2304.

[8] a) P. Cuniasse, L. C. Sowers, R. Eritja, B. Kaplan, M. F. Goodman, J. A. Cognet, M. LeBret,W. Guschlbauer,G. V. Fazakerley, Nucleic Acids Res. 1987, 15, $8003-8022$; b) P. Cuniasse, L. C. Sowers, R. Eritja, B. Kaplan, M. F. Goodman, J. A. Cognet, M. Le Bret, W. Guschlbauer, G. V. Fazakerley, Biochemistry 1989, 28, 2018 - 2026.

[9] a) L. Zhang, A. Peritz, E. Meggers, J. Am. Chem. Soc. 2005, 127, 4174 - 4175; b) M. K. Schlegel, A. E. Peritz, K. Kittigowittana, L. Zhang, E. Meggers, ChemBioChem 2007, 8, 927 - 932.

[10] G. te Velde, F. M. Bickelhaupt, E. J. Baerends, C. Fonseca Guerra, S. J. A. van Gisbergen, J. G. Snijders, T. Ziegler, J. Comput. Chem. 2001, 22, 931 - 967.

[11] a) J. Poater, M. Swart, C. Fonseca Guerra, F. M. Bickelhaupt, Chem. Commun. 2011, 47, 7326 - 7328; b) J. Poater, M. Swart, F. M. Bickelhaupt, C. Fonseca Guerra, Org. Biomol. Chem. 2014, 12, 4691 - 4700. 


\section{Glucose-nucleobase pseudo base pairs as a new binding motif in a DNA context}

Empar Vengut-Climent, Irene Gómez-Pinto, Ricardo Lucas, Pablo Peñalver, Anna

Aviñó, Célia Fonseca Guerra, F. Matthias Bickelhaupt, Ramón Eritja, Carlos González and Juan Carlos Morales

\section{Supplementary Information}

\section{Contents:}

Supplementary materials and methods

- Synthesis materials and methods 3

- Synthesis of $\mathbf{T}^{*}$, glc and 6dglc phosphoramidites 3-20

- ${ }^{1} \mathrm{H}$ and ${ }^{13} \mathrm{C}-\mathrm{NMR}$ spectra of new compounds 3-20

- Synthesis of natural and modified oligonucleotide DNA strands 20

- HPLC chromatograms of modified oligonucleotide DNA strands. 21-22

- Maldi-TOF mass of modified oligonucleotide DNA strands. 22

- Synthesis of oligonucleotide GNA strands 23

- HPLC chromatograms of oligonucleotide GNA strands. 24

- Maldi-TOF mass of oligonucleotide GNA strands. 24

- Thermal denaturation methods 25

- NMR methodology 25

- Structure Calculations 26

- DFT quantum chemical methods 26

Supplementary Figures

- Figure S1: Synthesis of flexible T* amidite. 27

- Figure S2: Melting curves for DNA double helices containing glc and 6dglc.28

- Figure S3. Melting curves for GNA double helices containing 6dglc. 29

- Figure S4. Imino region of the NMR spectra of helix 6dglc-G and helix 6dglcT. $\quad 30$

- Figure S5. More significant changes in proton chemical shifts along the sequence for helix 6dglc-G and helix 6dglc-T, 30-31

- Figure S6. Several regions of NOESY spectra of helix 6dglc-T 32

- Figure S7. Solution structure of helix 6dglc-T 33

- Figure S8. Solution structure of helix 6dglc-G 34

- Figure S9. Structures for pairs containing glc and 6dglc in vacuo and in water, computed at BLYP-D3(BJ)/TZ2P using COSMO to simulate aqueous solution. 
Supplementary Tables

- Table S1: ${ }^{1} \mathrm{H}-\mathrm{NMR}$ assignments of helix 6dglc-T. 36

- Table S2: ${ }^{1} \mathrm{H}-\mathrm{NMR}$ assignments of helix 6dglc-G. 37

- Table S3. Structurally relevant carbohydrate-DNA NOE contacts for helix 6dglc-G and helix 6dglc-T. 38

- Table S4. NMR structural constraints and calculation statistics. 39

- Table S5. Cartesian coordinates and ADF total energies of all stationary points in this study, computed at BLYP-D3(BJ)/TZ2P using COSMO to simulate aqueous solution. 


\section{Synthesis materials and methods}

All chemicals were obtained from chemical suppliers (Sigma-Aldrich, Carbosynth) and used without further purification, unless otherwise noted. Anhydrous dichloromethane was dried in an aluminium oxide column machine, PURESOLV (Scharlab). Other anhydrous solvents were dried over molecular sieves ( $4 \AA$ ) for $48 \mathrm{~h}$.

All reactions were monitored by TLC on precoated silica gel 60 plates $\mathrm{F}_{254}$ (Merck) and detected by heating after staining with $\mathrm{H}_{2} \mathrm{SO}_{4}: \mathrm{EtOH}(1: 9, v / v)$, anisaldehyde (450 ml ethanol, 25 $\mathrm{ml}$ anisaldehyde, $25 \mathrm{ml} \mathrm{H}_{2} \mathrm{SO}_{4}$ and $\left.1 \mathrm{ml} \mathrm{AcOH}\right)$ or Mostain $\left(500 \mathrm{ml}\right.$ of $10 \% \mathrm{H}_{2} \mathrm{SO}_{4}, 25 \mathrm{~g}$ of $\left.\left(\mathrm{NH}_{4}\right)_{6} \mathrm{Mo}_{7} \mathrm{O}_{24} \bullet 4 \mathrm{H}_{2} \mathrm{O}, 1 \mathrm{~g} \mathrm{Ce}\left(\mathrm{SO}_{4}\right)_{2} \bullet 4 \mathrm{H}_{2} \mathrm{O}\right)$. Products were purified by flash chromatography with silica gel 60 (200-400 mesh). Eluents are indicated for each particular case.

NMR spectra were recorded on either a Bruker Avance 300 or ARX $400 \mathrm{MHz}$ or Bruker Avance DRX $500 \mathrm{MHz}\left[300,400\right.$ or $500 \mathrm{MHz}\left({ }^{1} \mathrm{H}\right), 75,100,125 \mathrm{MHz}\left({ }^{13} \mathrm{C}\right)$, at room temperature for solutions in $\mathrm{CDCl}_{3}$ or $\mathrm{CD}_{3} \mathrm{OD}$ ] spectrometer. Chemical shifts are referred to the solvent signal and are expressed in ppm. 2D NMR experiments (COSY, TOCSY and HMQC) were carried out when necessary to assign the corresponding signals of the new compounds. Low resolution electrospray mass spectral analyses were obtained on a Bruker Esquire 6000 ion trap mass spectrometer. High resolution FAB (+) mass spectral analyses were obtained on a Micromass AutoSpec-Q spectrometer.

\section{Synthesis of T*, glc and 6dglc phosphoramidites}

\section{(S)-2,2-dimethyl-4-(2,3,4,6-tetra- $O$-acetyl- $\beta$-D-glucopyranos-1-ylmethyl )dioxolane (4)}

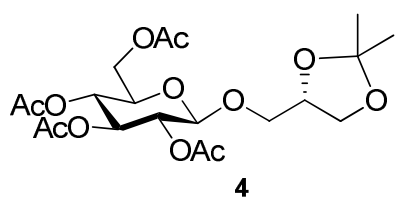

To a solution of 2,3,4,6-tetra- $O$-acetyl- $\alpha, \beta$-D-glucopyranosyl trichloroacetimidate 1 (3 $\mathrm{g}, 6.08 \mathrm{mmol})$ and $(S)-(+)-1,2$-isopropylideneglycerol $3(2.26 \mathrm{ml}, 18.26 \mathrm{mmol})$ in anhydrous $\mathrm{CH}_{2} \mathrm{Cl}_{2}(40 \mathrm{ml}) \mathrm{BF}_{3} . \mathrm{OEt}_{2}(77 \mu \mathrm{l}, 0.609 \mathrm{mmol})$ was added. The reaction was then stirred for 30 min and $\mathrm{NEt}_{3}(0.5 \mathrm{ml})$ was then added. The solvent were removed and the crude was purified by flash column chromatography (Hex/EtOAc, from 2:1 to 1:2) to afford 4 (2415 mg, 86\%); ${ }^{1} \mathrm{H}$ NMR (400 MHz, $\left.\mathrm{CDCl}_{3}\right) \delta(\mathrm{ppm}): 5.17\left(\mathrm{t}, J=9.44 \mathrm{~Hz}, 1 \mathrm{H}, \mathrm{H}_{3}\right), 5.04\left(\mathrm{t}, J=9.60 \mathrm{~Hz}, 1 \mathrm{H}, \mathrm{H}_{4}\right)$, $4.96\left(\mathrm{t}, J=9.20 \mathrm{~Hz}, 1 \mathrm{H}, \mathrm{H}_{2}\right), 4.59\left(\mathrm{~d}, J=7.96 \mathrm{~Hz}, 1 \mathrm{H}, \mathrm{H}_{1}\right), 4.25-4.19\left(\mathrm{~m}, 2 \mathrm{H}, \mathrm{H}_{6},-\mathrm{OCH} H-\right.$ isopropylidene), $4.10\left(\mathrm{dd}, J=1.6 / 12.0 \mathrm{~Hz}, 1 \mathrm{H}, \mathrm{H}_{6}\right), 3.99-3.96\left(\mathrm{~m}, 1 \mathrm{H},-\mathrm{CH}_{-}\right.$isopropylidene), 3.84 (dd, $J$ $=4.4 / 10.8 \mathrm{~Hz}, 1 \mathrm{H},-\mathrm{OCH}_{-}$-isopropylidene $), 3.78-3.74(\mathrm{~m}, 1 \mathrm{H},-\mathrm{OC} H \mathrm{H}-), 3.70-3.68\left(\mathrm{~m}, 1 \mathrm{H}, \mathrm{H}_{5}\right)$, 
3.63-3.60 (dd, $J=5.8 / 10.6 \mathrm{~Hz}, 1 \mathrm{H},-\mathrm{OCH} H-), 2.06,2.02,2.00,1.98\left(4 \mathrm{~s}, 12 \mathrm{H}, 3 \mathrm{x}-\mathrm{OCOCH}_{3}\right)$, $1.41,1.33\left(2 \mathrm{~s}, 6 \mathrm{H}, 2 \mathrm{x}-\mathrm{C}\left(\mathrm{CH}_{3}\right)_{2}\right) ;{ }^{13} \mathrm{C} \mathrm{NMR}\left(100 \mathrm{MHz}, \mathrm{CDCl}_{3}\right): \delta=170.6,170.2,169.4,169.3$ (CO), $109.3\left(-C\left(\mathrm{CH}_{3}\right)_{2}\right), 100.9\left(\mathrm{C}_{1}\right), 74.3,72.7,71.8,71.1,69.1,68.4,66.1,61.9,26.7,25.1$, 20.7, 20.6, 20.5. HRMS (FAB ${ }^{+}$) Calcd. for $\mathrm{C}_{20} \mathrm{H}_{30} \mathrm{NaO}_{12}(\mathrm{M}+\mathrm{Na})$ : 485.1635, found; 485.1622 .

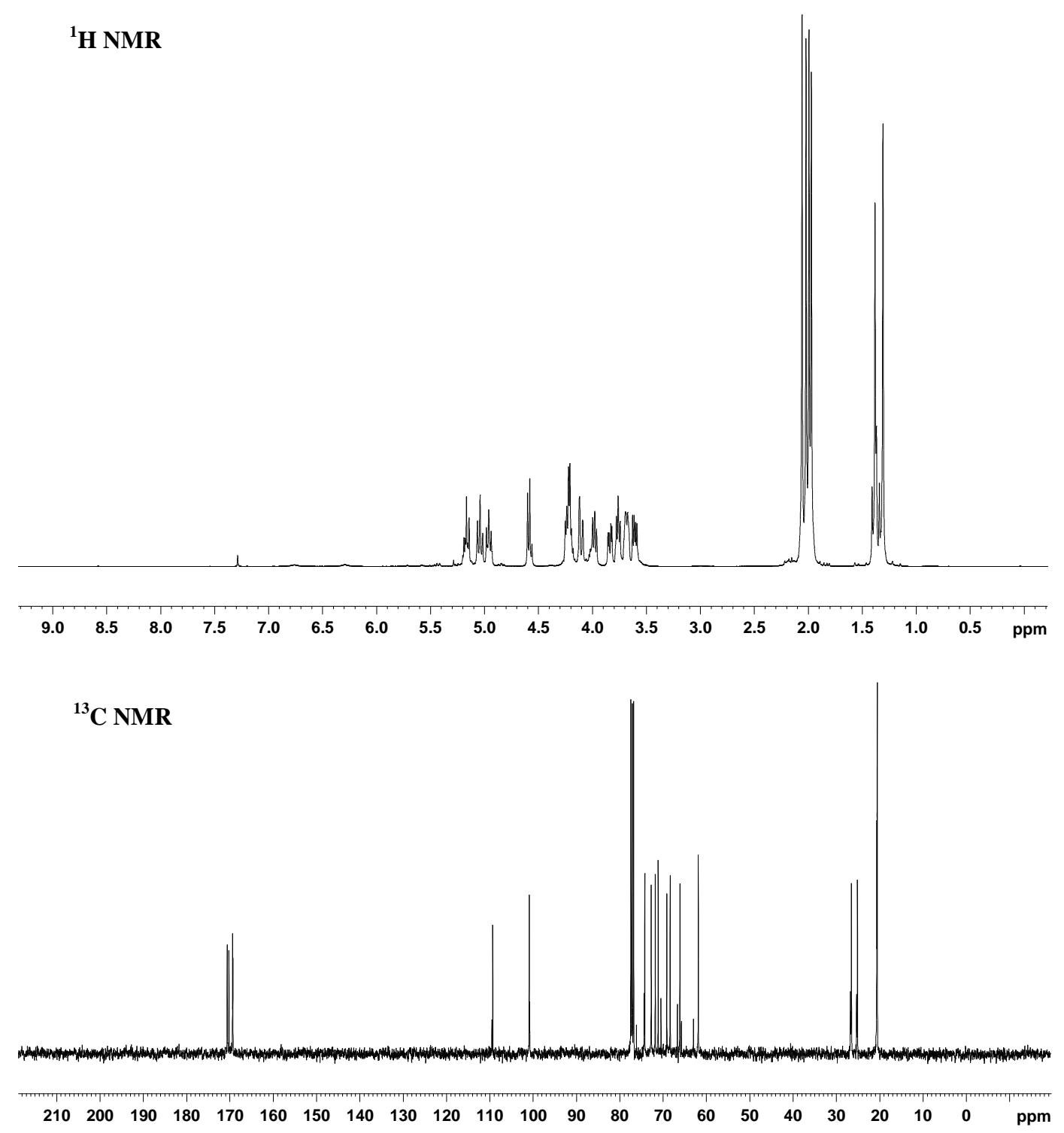

(S)-2,2-dimethyl-4-(2,3,4,-tri-O-acetyl-6-deoxy- $\beta$-D-glucopyranos-1-ylmethyl)dioxolane (5)

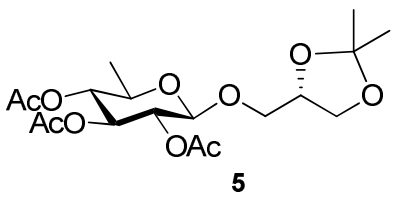


To a solution of 2,3,4-tri- $O$-acetyl-6-deoxyl- $\beta$-D-glucopyranosyl trichloroacetimidate $\mathbf{1}$ (900 mg, $2.07 \mathrm{mmol})$ and (S)-(+)-1,2-isopropylideneglycerol $3(388 \mu \mathrm{l}, 3.10 \mathrm{mmol})$ in anhydrous $\mathrm{CH}_{2} \mathrm{Cl}_{2}(10 \mathrm{ml}) \mathrm{BF}_{3} . \mathrm{OEt}_{2}(30 \mu \mathrm{l}, 0.21 \mathrm{mmol})$ was added. The reaction was stirred for $30 \mathrm{~min}$ and $\mathrm{NEt}_{3}(0.5 \mathrm{ml})$ was then added. The solvent was removed and the crude was purified by flash column chromatography (Hex/EtAcO, from 3:1 to 1:1) to afford 5 (500 mg, 60\%); ${ }^{1} \mathrm{H}$ $\operatorname{NMR}\left(500 \mathrm{MHz}, \mathrm{CDCl}_{3}\right) \delta(\mathrm{ppm}): 5.13\left(\mathrm{t}, J=9.5 \mathrm{~Hz}, 1 \mathrm{H}, \mathrm{H}_{3}\right), 4.94\left(\mathrm{t}, J=8.0 \mathrm{~Hz}, 1 \mathrm{H}, \mathrm{H}_{2}\right), 4.78$ (t, $\left.J=9.5 \mathrm{~Hz}, 1 \mathrm{H}, \mathrm{H}_{4}\right), 4.54\left(\mathrm{~d}, J=8.0 \mathrm{~Hz}, 1 \mathrm{H}, \mathrm{H}_{1}\right), 4.24-4.20\left(\mathrm{~m}, 1 \mathrm{H},-\mathrm{CH}_{\text {-isopropylidene }}\right.$ ), 3.99-3.97 (m, 1H, -OCHH-), 3.84 (dd, $J=4.5 / 10.5 \mathrm{~Hz}, 1 \mathrm{H},-\mathrm{OCH} H-), 3.76$ (dd, $J=6.0 / 8.0 \mathrm{~Hz}, 1 \mathrm{H},-$ $\mathrm{OC} H \mathrm{H}-)$, 3.60-3.53 (m, 2H, $\left.\mathrm{H}_{5},-\mathrm{OCH} H-\right), 2.01,2.00,1.97$ (3s, 9H, 3x-OCOCH$\left.H_{3}\right), 1.38,1.31$ $\left(2 \mathrm{~s}, 6 \mathrm{H},-\mathrm{C}\left(\mathrm{CH}_{3}\right)_{2}\right), 1.22-1.20\left(\mathrm{~m}, 3 \mathrm{H},-\mathrm{CH}_{3}\right) ;{ }^{13} \mathrm{C} \mathrm{NMR}\left(125 \mathrm{MHz}, \mathrm{CDCl}_{3}\right) \delta(\mathrm{ppm}): 170.3$,

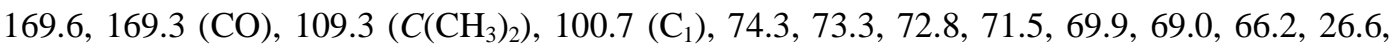
25.2, 20.6, 17.3. HRMS $\left(\mathrm{FAB}^{+}\right)$Calcd. for $\mathrm{C}_{18} \mathrm{H}_{30} \mathrm{O}_{10} \mathrm{Na}(\mathrm{M}+\mathrm{Na})$ : 427.1580, found; 427.1588.

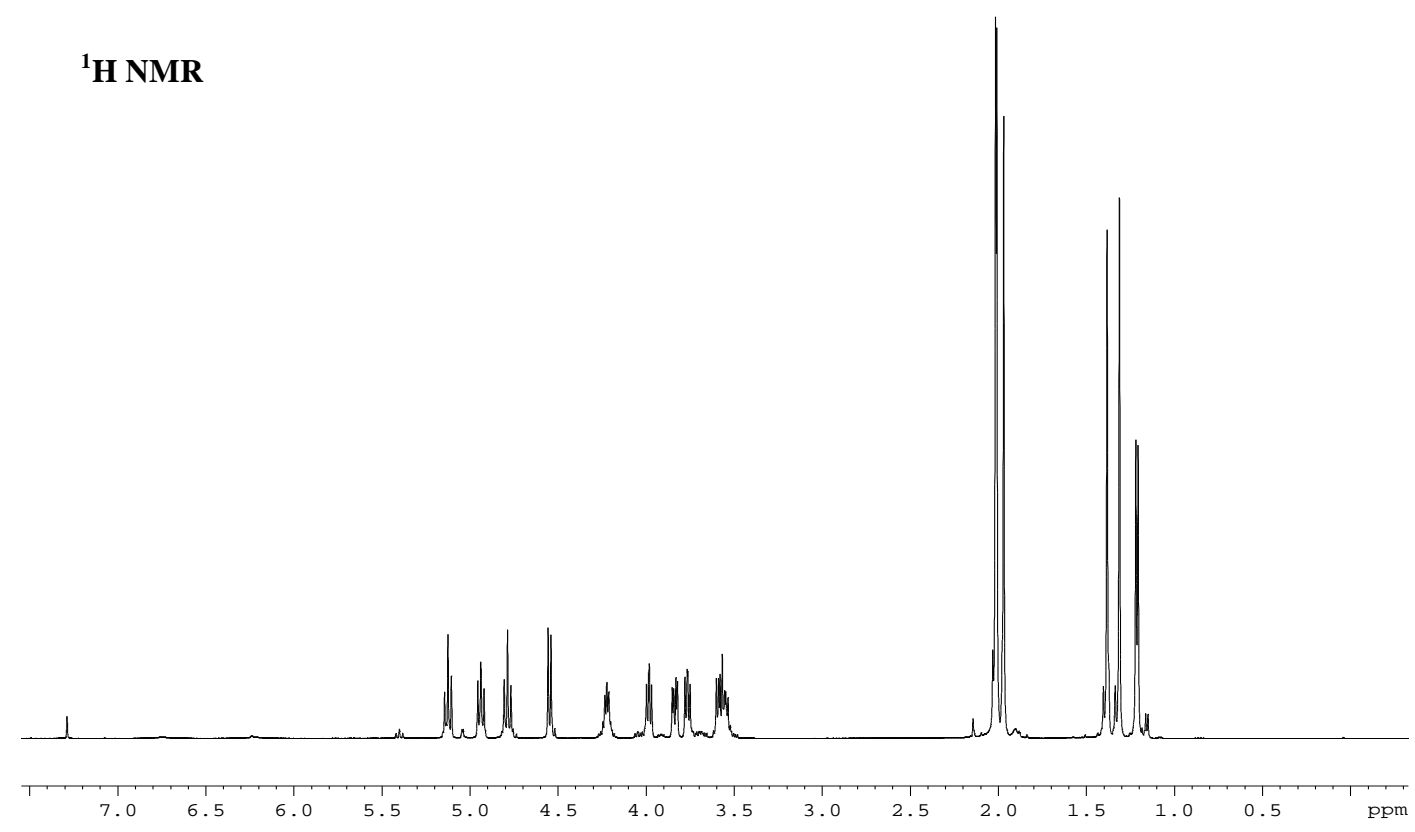




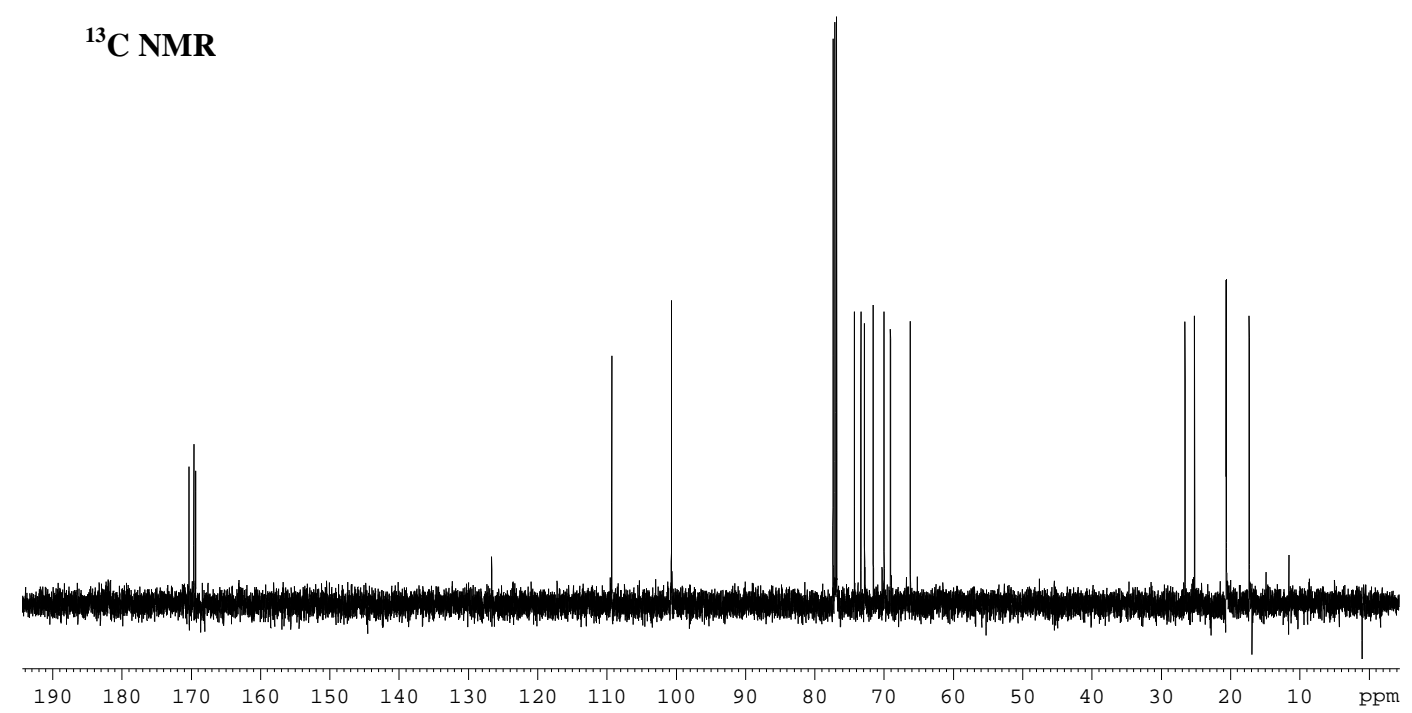

(S)-2,2-dimethyl-4-( $N$-benzoylthymin-1-ylethyl)dioxolane (13)

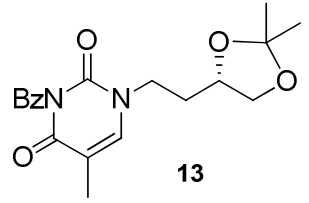

To a solution of the benzoylated thymine $\mathbf{1 1}^{1}(600 \mathrm{mg}, 2.61 \mathrm{mmol})$ in dry DMF (10 ml), $\mathrm{K}_{2} \mathrm{CO}_{3}(396 \mathrm{mg}, 2.87 \mathrm{mmol})$ and TBAI $(96 \mathrm{mg}, 0.261 \mathrm{mmol})$ were added. Then, (S)-toluene-4sulfonic acid 2-(2,2'-dimethyl-[1,3]dioxolan-4-yl)-ethyl ester $\mathbf{1 2}^{2}$ was added and the reaction mixture was stirred at $70^{\circ} \mathrm{C}$ for 2 hours and finally at room temperature overnight. The reaction mixture was washed with water and saturated $\mathrm{NH}_{4} \mathrm{Cl}$, and extracted with ethyl acetate and ether. Solvents were removed and the crude was purified by silica gel column chromatography (Hex/EtOAc from 3:1 to 0:1) to give compound 13 (498 mg, $53 \%$ ) as a white foam. ${ }^{1} \mathrm{H}$ NMR (400 MHz, $\left.\mathrm{CDCl}_{3}\right) \delta(\mathrm{ppm}): 7.90\left(\mathrm{~d}, J=7.2 \mathrm{~Hz}, 2 \mathrm{H}, \mathrm{H}_{\text {benzoyl }}\right), 7.62\left(\mathrm{t}, J=7.2 \mathrm{~Hz}, 1 \mathrm{H}, \mathrm{H}_{\text {benzoyl }}\right)$, 7.47 (t, $\left.J=7.2 \mathrm{~Hz}, 2 \mathrm{H}, \mathrm{H}_{\text {benzoyl }}\right), 7.17$ (s, $\left.1 \mathrm{H}, \mathrm{H}_{\text {thymine }}\right), 4.13-4.03\left(\mathrm{~m}, 2 \mathrm{H},-\mathrm{OCH} H\right.$-isopropylidene, $_{\text {- }}$ $\mathrm{CH}_{\text {isopropylidene }}$ ), $4.00-3.94\left(\mathrm{~m}, 1 \mathrm{H},-\mathrm{CH} H-\mathrm{CH}_{2} \mathrm{~N}-\right.$ ) $, 3.79-3.72\left(\mathrm{~m}, 1 \mathrm{H},-\mathrm{CH} \mathrm{H}-\mathrm{CH}_{2} \mathrm{~N}-\right), 3.56-3.53$ (m, $1 \mathrm{H},-\mathrm{CH} \mathrm{H}_{-}$isopropylidene), 2.02-1.95 (m, $1 \mathrm{H},-\mathrm{CH}_{2}-\mathrm{CH} H-\mathrm{CH}-\mathrm{O}-$ ), 1.93 (s, 3H,- $\mathrm{CH}_{3 \text { thymine }}$ ), 1.871.79 (m, 1H, - $\mathrm{CH}_{2}$ - $\left.\mathrm{CHH}-\mathrm{CH}-\mathrm{O}-\right), 1.40$ (s, 3H, - $\mathrm{CH}_{3 \text { isopropylidene }}$ ), 1.33 (s, $3 \mathrm{H},-\mathrm{CH}_{3 \text { isopropylidene); }}{ }^{13} \mathrm{C}$ $\operatorname{NMR}\left(100 \mathrm{MHz}, \mathrm{CDCl}_{3}\right) \delta$ (ppm): 169.1, 163.2, 149.8, 140.9, 135.0, 131.7, 130.4, 129.1, 110.4, 109.3, 76.8, 72.9, 69.1, 46.4, 32.5, 27.0, 25.5, 12.3. HRMS $\left(\mathrm{ES}^{+}\right)$Calcd. for $\mathrm{C}_{16} \mathrm{H}_{18} \mathrm{~N}_{2} \mathrm{O}_{5}$ : 358.1529 , found; 358.1518 . 

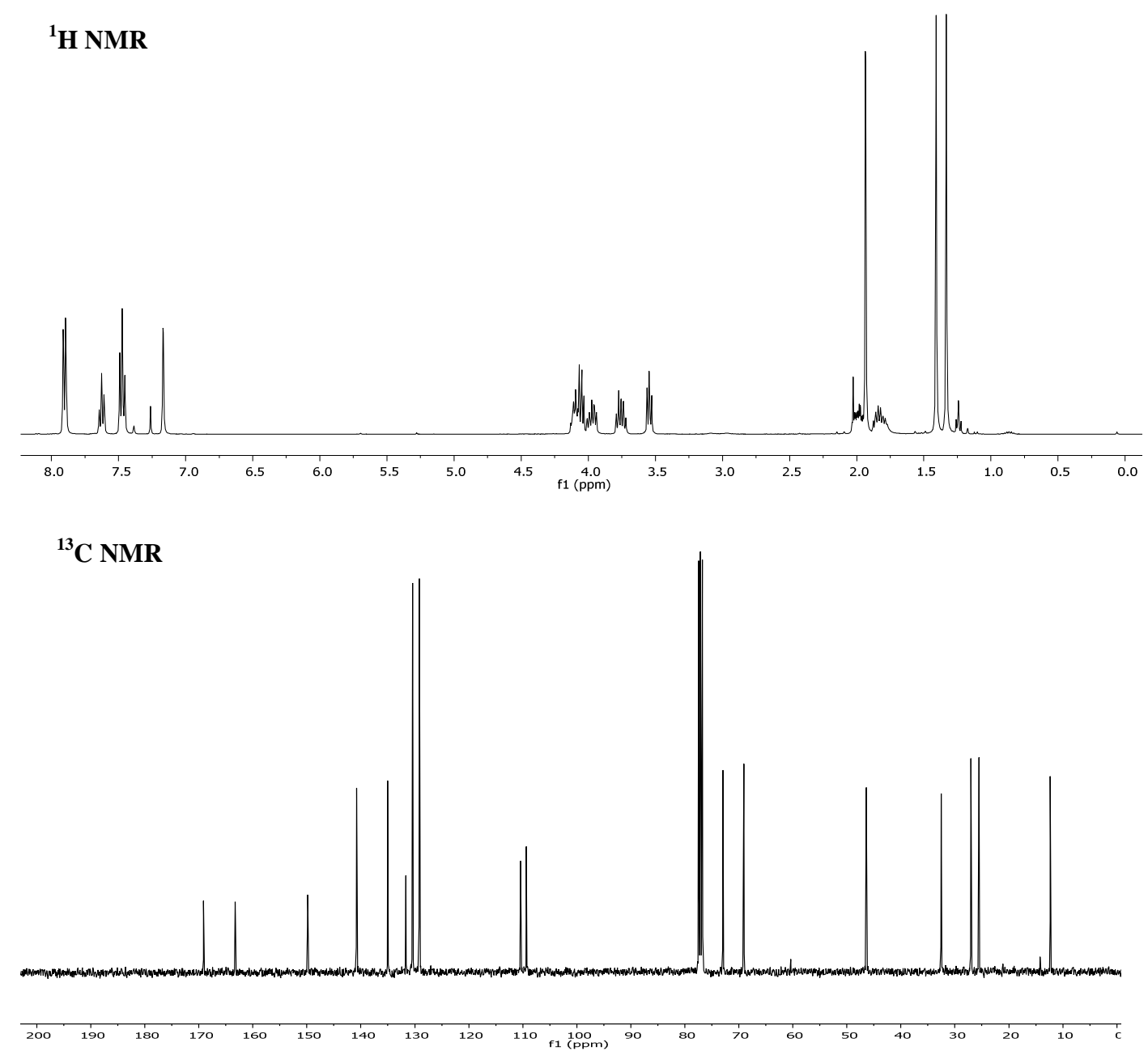

General procedure for isopropylidene removal. A solution of the 2,2dimethyldioxolane derivative $(1.93 \mathrm{mmol})$ was dissolved in $40 \mathrm{ml}$ of acetic acid $80 \%$ and the solution was stirred at $80^{\circ} \mathrm{C}$ for 1 to 2 hours. The solvent was removed and coevaporated with toluene. The reaction crude was then purified by flash chromatography.

(R)-2,3-dihydroxypropyl 2,3,4,6-tetra- $O$-acetyl- $\beta$-D-glucopyranoside (17)

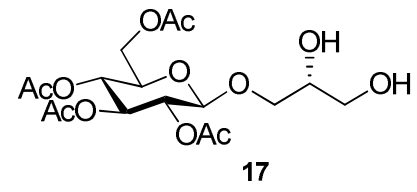

Compound 4 (3.5 g, $7.56 \mathrm{mmol})$ was dissolved in a mixture of $\mathrm{CH}_{3} \mathrm{COOH} / \mathrm{H}_{2} \mathrm{O}(150 \mathrm{ml}, 4 ; 1)$. The reaction mixture was reacted following the general isopropylidene removal procedure. The 
crude was purified by silica gel column chromathography (EtOAc/MeOH, from 1:0 to 10:1) to give $17(2.8 \mathrm{~g}, 87 \%)$ as a syrup. ${ }^{1} \mathrm{H} \mathrm{NMR}\left(400 \mathrm{MHz}, \mathrm{CDCl}_{3}\right) \delta(\mathrm{ppm}): 5.15(\mathrm{t}, J=9.3 \mathrm{~Hz}, 1 \mathrm{H}$, $\left.\mathrm{H}_{3}\right), 5.02\left(\mathrm{t}, J=9.5 \mathrm{~Hz}, 1 \mathrm{H}, \mathrm{H}_{4}\right), 4.93\left(\mathrm{t}, J=9.3 \mathrm{~Hz}, 1 \mathrm{H}, \mathrm{H}_{2}\right), 4.48\left(\mathrm{~d}, J=7.8 \mathrm{~Hz}, 1 \mathrm{H}, \mathrm{H}_{1}\right), 4.13$ (s, $2 \mathrm{H}, \mathrm{H}_{6}, \mathrm{H}_{6^{\prime}}$ ), 3.79-3.55 (m, 6H, -CH-, 2x-OCH $2^{-}, \mathrm{H}_{5}$ ), 2.57 (br.s, 1H, OH), 2.03, 2.00, 1.97, 1.94 $\left(4 \mathrm{~s}, 12 \mathrm{H}, 3 \mathrm{x}-\mathrm{OCOCH}_{3}\right) ;{ }^{13} \mathrm{C}$ NMR $\left(101 \mathrm{MHz}, \mathrm{CD}_{3} \mathrm{CD}\right) \delta(\mathrm{ppm}): 171.0,170.3,170.0,169.9$ (CO), $100.8\left(\mathrm{C}_{1}\right), 72.8,71.5,71.4,70.6,70.5,68.4,62.8,62.7,61.7,19.3,19.2 . \mathrm{HRMS}_{\left(\mathrm{FAB}^{+}\right)}$ Calcd. for $\mathrm{C}_{17} \mathrm{H}_{26} \mathrm{NaO}_{12}(\mathrm{M}+\mathrm{Na})$ : 445.1322, found; 445.1329 .

${ }^{1}$ H NMR

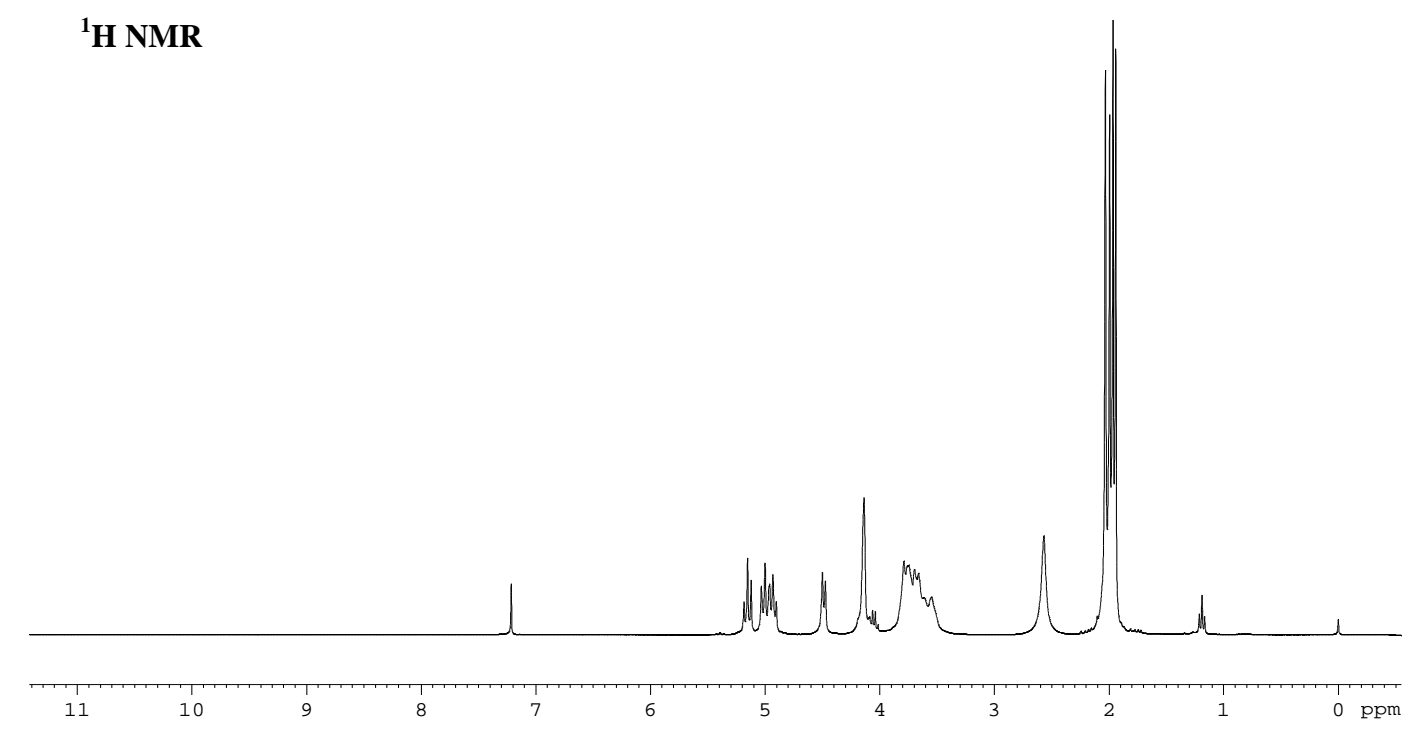

${ }^{13}$ C NMR

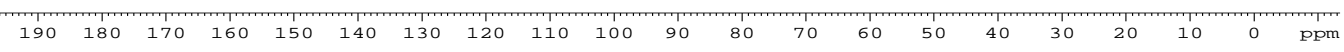


(R)-2,3-dihydroxypropyl 2,3,4,-tri- $O$-acetyl-6-deoxyl- $\beta$-D-glucopyranoside (18)

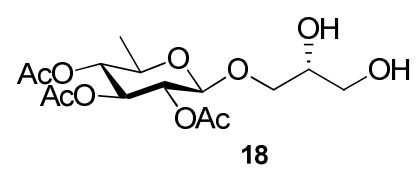

Compound 5 ( $1 \mathrm{~g}, 2.45 \mathrm{mmol})$ was dissolved in a mixture of $\mathrm{CH}_{3} \mathrm{COOH}(18 \mathrm{ml})$ and $\mathrm{H}_{2} \mathrm{O}(2 \mathrm{ml})$. The reaction mixture was reacted following the general isopropylidene removal procedure. The crude was purified by silica gel column chromathography using as eluent (EtOAc:MeOH, 1:0-10:1) to give $\mathbf{1 8}(599 \mathrm{mg}, 68 \%)$ as a syrup. ${ }^{1} \mathrm{H} \mathrm{NMR}\left(\mathrm{CDCl}_{3}, 400 \mathrm{MHz}\right.$,) $\delta$ (ppm): 5.14 (t, $J=9.6 \mathrm{~Hz}, 1 \mathrm{H}, \mathrm{H}_{3}$ ), 4.94 (dd, $J=8.0 / 9.6 \mathrm{~Hz}, 1 \mathrm{H}, \mathrm{H}_{2}$ ), 4.79 (t, $J=9.6 \mathrm{~Hz}, 1 \mathrm{H}, \mathrm{H}_{4}$ ), 4.49 (d, $\left.J=8.0 \mathrm{~Hz}, 1 \mathrm{H}, \mathrm{H}_{1}\right), 3.85-3.79\left(\mathrm{~m}, 2 \mathrm{H},-\mathrm{OCH}_{2}\right)$, 3.72-3.52 (m, 4H, $\left.\mathrm{H}_{5},-\mathrm{CH}-,-\mathrm{OCH}_{2}-\right)$, 3.40 (br.s, 1H, OH), 2.84 (br. s, 1H, OH), 2.03, 2.01, 1.98 (3s, 9H, 3 x - $\left.\mathrm{OCOCH}_{3}\right), 1.21$ (m, 3H, $\left.\mathrm{CH}_{3}\right) ;{ }^{13} \mathrm{C} \mathrm{NMR}\left(\mathrm{CDCl}_{3}, 125 \mathrm{MHz}\right) \delta(\mathrm{ppm}): 170.3,169.8,169.7(\mathrm{CO}), 100.9\left(\mathrm{C}_{1}\right), 73.2,72.6$, 71.9, 71.6, 70.4, 70.0, 63.4, 20.7, 20.6, 17.2. HRMS $\left(\mathrm{FAB}^{+}\right)$Calcd. for $\mathrm{C}_{15} \mathrm{H}_{24} \mathrm{NaO}_{10}(\mathrm{M}+\mathrm{Na})$ : 387.1267 , found; 387.1257 .

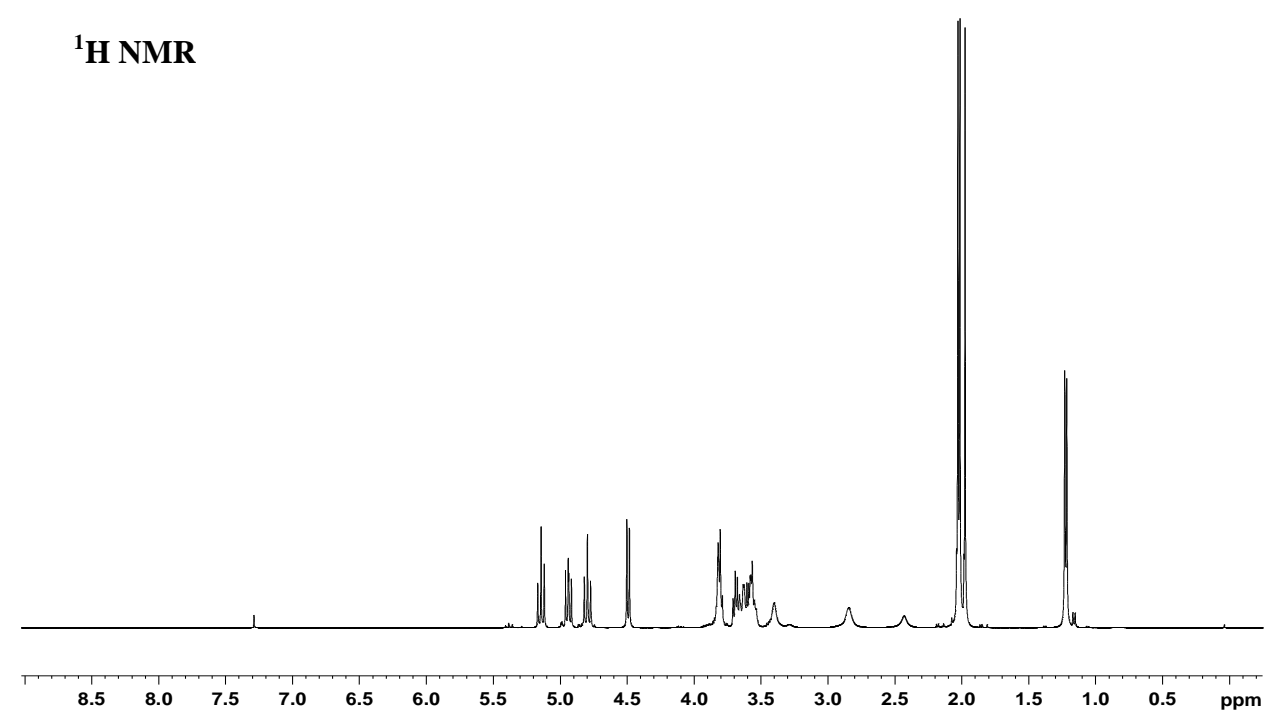




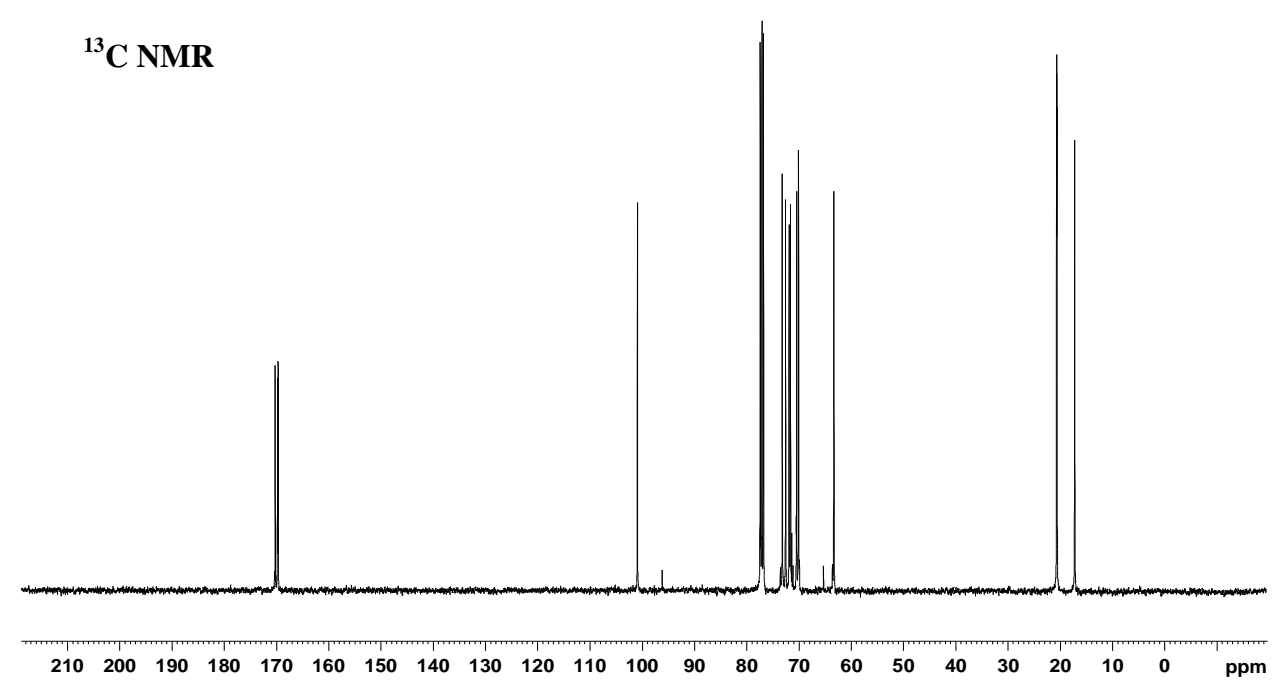

(S)-l-(3,4-dihydroxybutyl)- $N$-benzoylthymine (14)

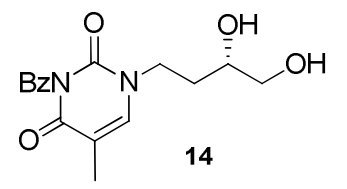

A solution of compound $\mathbf{1 3}(1.45 \mathrm{~g}, 1.93 \mathrm{mmol})$ was dissolved in $40 \mathrm{ml}$ of acetic acid $80 \%$ and was reacted following the general isopropylidene removal procedure. Flash chromatography was used (Hex/EtOAc from 1:6 to 0:1 and EtOAc/MeOH from 20:1 to 10:1) to give compound $14(72 \%)$ as a white solid. ${ }^{1} \mathrm{H}$ NMR (400 MHz, $\left.\mathrm{CDCl}_{3}\right) \delta(\mathrm{ppm}): 7.91$ (d, $J=7.2$ $\left.\mathrm{Hz}, 2 \mathrm{H}, \mathrm{H}_{\text {benzoyl }}\right), 7.68-7.64\left(\mathrm{~m}, 1 \mathrm{H}, \mathrm{H}_{\text {benzoyl }}\right), 7.52-7.48\left(\mathrm{~m}, 2 \mathrm{H}, \mathrm{H}_{\text {benzoyl }}\right), 7.16\left(\mathrm{~s}, 1 \mathrm{H}, \mathrm{H}_{\text {thymine }}\right)$, 4.16-4.03 (m, 1H, -OCHH-), 3.88-3.70 (m, 2H, -OCHH-, - $\mathrm{CH}_{-}$- $_{\text {isopropylidene }}$ ), 3.60-3.51 (m, 2H, $\mathrm{CH}_{2}-\mathrm{CH}_{2} \mathrm{~N}-$ ), 2.20 (s, 3H, $-\mathrm{CH}_{3 \text { thymine }}$ ), 2.15-1.75 (m, $\left.2 \mathrm{H},-\mathrm{NCH}_{2} \mathrm{CH}_{2} \mathrm{O}-\right) ;{ }^{13} \mathrm{C} \mathrm{NMR}(100 \mathrm{MHz}$, $\left.\mathrm{CDCl}_{3}\right) \delta(\mathrm{ppm}): 172.2,165.0,149.8,140.9,136.1,132.7,130.4,129.1,110.4,68.4,66.3,43.4$, 31.5, 12.3. HRMS $\left(\mathrm{FAB}^{+}\right)$Calcd. for $\mathrm{C}_{16} \mathrm{H}_{18} \mathrm{NaO}_{2}(\mathrm{M}+\mathrm{Na})$ : 341.1113, found: 341.1109 .

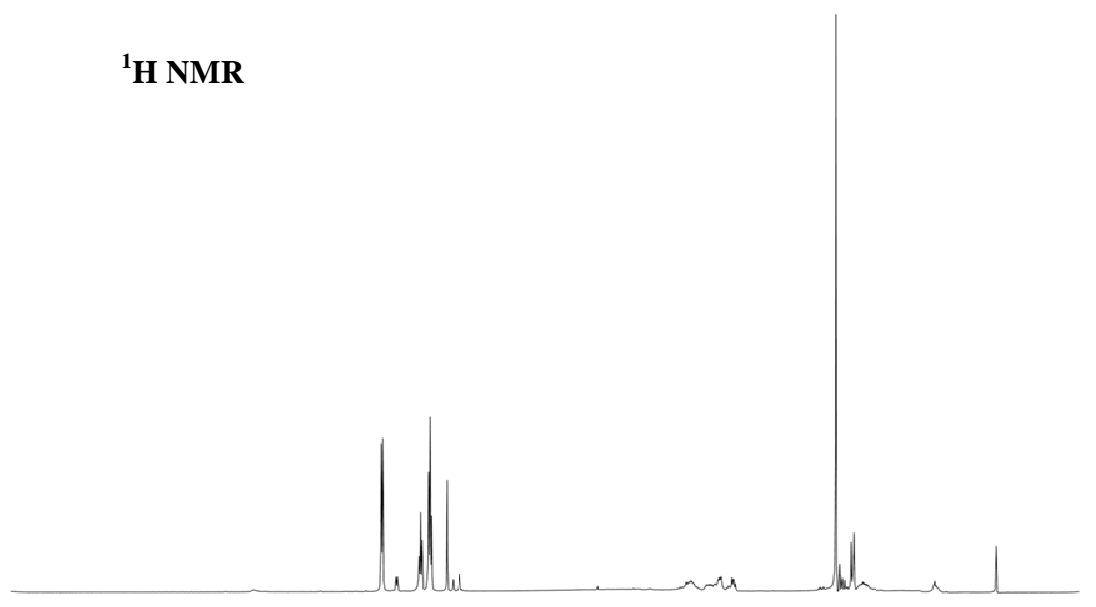




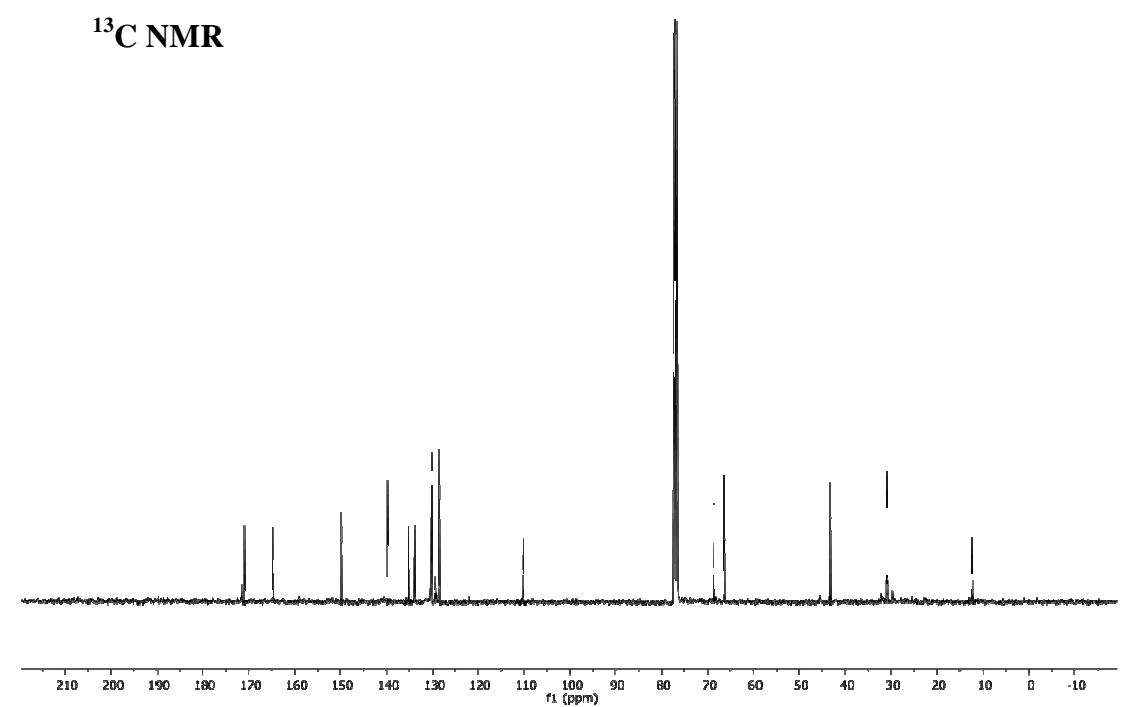

General procedure for primary hydroxyl protection with 4,4-dimethoxytritylgroup. DIPEA (1.941 mmol) and DMAP $(0.097 \mathrm{mmol})$ were added to a solution of the unprotected compound $(0.971 \mathrm{mmol})$ in pyridine $/ \mathrm{CH}_{2} \mathrm{Cl}_{2}(1: 1,10 \mathrm{ml})$. The temperature was lowered to $0{ }^{\circ} \mathrm{C}$ and DMTCl $(1.456 \mathrm{mmol})$ was slowly added. The reaction was stirred for 15 minutes at $0{ }^{\circ} \mathrm{C}$ and $3 \mathrm{~h}$ at room temperature. Then, reaction was stopped by adding $\mathrm{MeOH}$ and the solvent was removed in vacuo. The reaction mixture was purified by flash chromatography.

\section{(R)-3-(4,4'-dimethoxytrityloxy)-2-hydroxypropyl 2,3,4,6-tetra- $O$-acetyl- $\beta$-D-}

glucopyranoside (6)

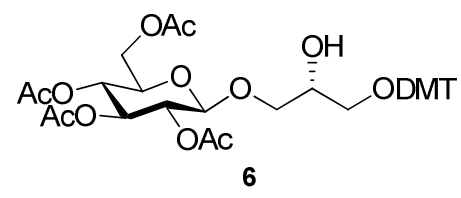

A solution of compound $17(400 \mathrm{mg}, 0.95 \mathrm{mmol})$ in dry pyridine- $\mathrm{CH}_{2} \mathrm{Cl}_{2}(1: 1,6 \mathrm{ml})$ was reacted following the general procedure for hydroxyl protection. The crude was purified by silica gel column chromathography (Hex/EtOAc, from 2:1 to 1:2) to give 6 (484 mg, 70\%) as a syrup. ${ }^{1} \mathrm{H}$ NMR $\left(500 \mathrm{MHz}, \mathrm{CDCl}_{3}\right) \delta(\mathrm{ppm}): 7.42\left(\mathrm{~d}, 2 \mathrm{H}, J=7.8 \mathrm{~Hz}, \mathrm{H}_{\text {arom }}\right), 7.33-7.17(\mathrm{~m}, 7 \mathrm{H}$, $\mathrm{H}_{\text {arom }}$ ), $6.83\left(\mathrm{~d}, 4 \mathrm{H}, J=8.7 \mathrm{~Hz}, \mathrm{H}_{\text {arom }}\right), 5.20\left(\mathrm{t}, 1 \mathrm{H}, J=9.6 \mathrm{~Hz}, \mathrm{H}_{3}\right), 5.08\left(\mathrm{t}, 1 \mathrm{H}, J=9.6 \mathrm{~Hz}, \mathrm{H}_{4}\right)$, 4.99 (t, $\left.1 \mathrm{H}, J=9.3 \mathrm{~Hz}, \mathrm{H}_{2}\right), 4.55\left(\mathrm{~d}, 1 \mathrm{H}, J=7.8 \mathrm{~Hz}, \mathrm{H}_{1}\right), 4.22-4.11\left(\mathrm{~m}, 2 \mathrm{H}, \mathrm{H}_{6}, \mathrm{H}_{6}\right)$, 3.94-3.77 (m, 9H,- $\left.-\mathrm{OCH}_{2^{-}}, 2 \mathrm{x}-\mathrm{OCH}_{3},-\mathrm{CH}-\right), 3.74-3.71\left(\mathrm{~m}, 1 \mathrm{H}, \mathrm{H}_{5}\right), 3.20-3.15\left(\mathrm{~m}, 2 \mathrm{H},-\mathrm{OCH}_{2}-\right), 2.06$, 2.04, 2.01, $\left.1.97\left(4 \mathrm{~s}, 12 \mathrm{H}, 4 \mathrm{x}-\mathrm{OCOCH} \mathrm{H}_{3}\right) ;{ }^{13} \mathrm{C} \mathrm{NMR} \mathrm{(100} \mathrm{MHz}, \mathrm{CDCl}_{3}\right): \delta$ (ppm): 170.7, 170.2, 
169.5, 167.9 (CO), 158.5, 144.8, 135.9, 134.1, 131.8, 130.0, 129.1, 128.1, 127.8, 126.8, 123.5, 113.2, 101.3 $\left(\mathrm{C}_{1}\right), 86.0,73.1,72.7,71.9,71.3,70.8,69.9,68.4,55.2,44.7,20.7,20.6$. HRMS $\left(\mathrm{FAB}^{+}\right)$Calcd. for $\mathrm{C}_{38} \mathrm{H}_{44} \mathrm{NaO}_{14}(\mathrm{M}+\mathrm{Na})$ : 747.2629, found; 747.2639.

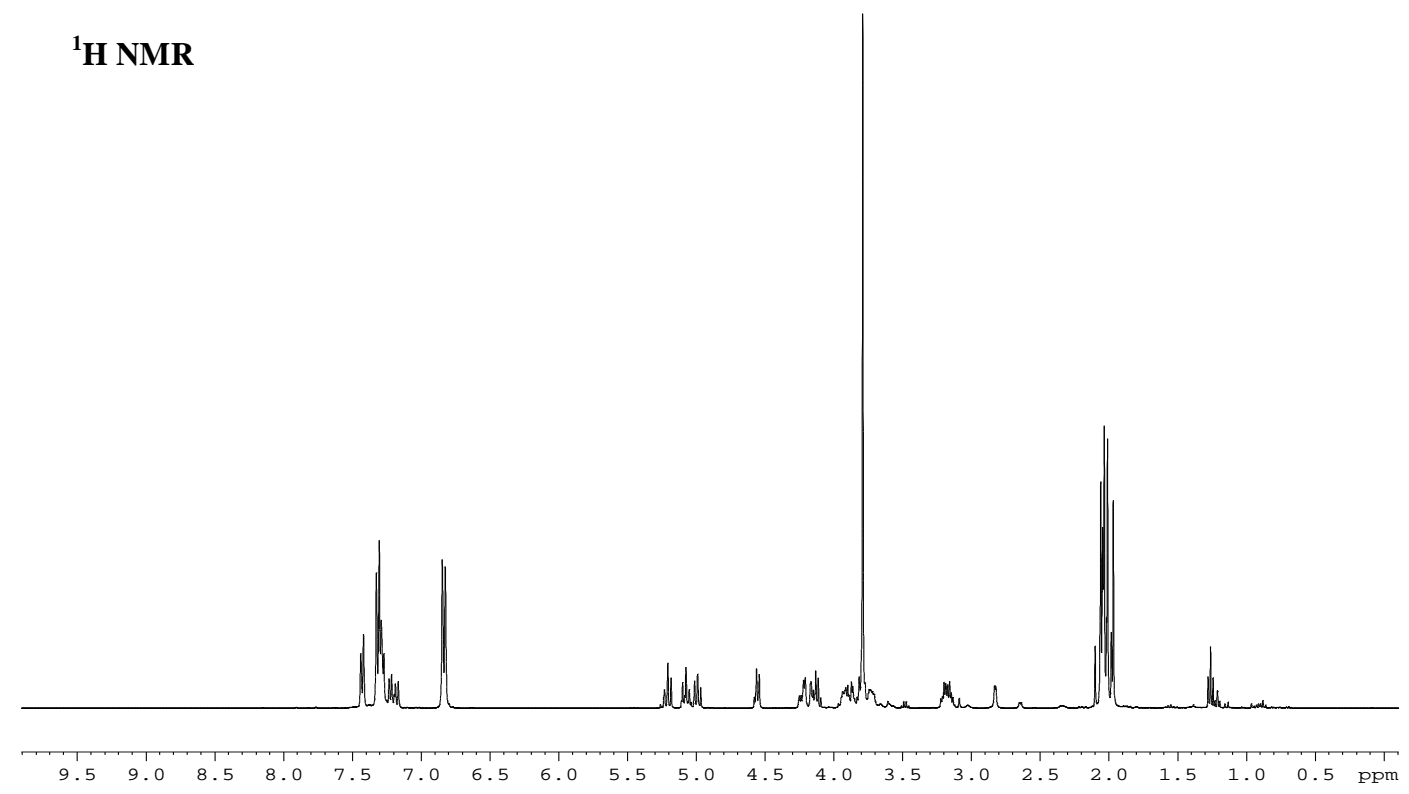

${ }^{13}$ C NMR

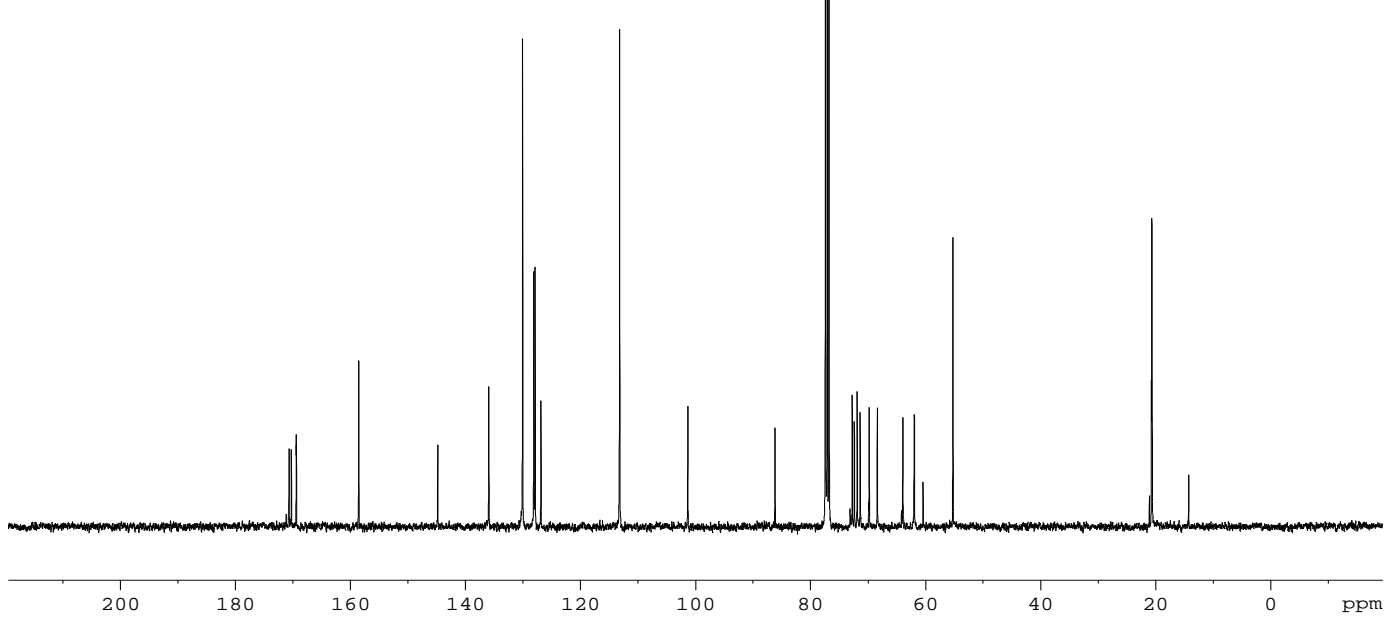

(R)-3-(4,4'-dimethoxytrityloxy)-2-hydroxypropyl 2,3,4-tri- $O$-acetyl-6-deoxyl- $\beta$-Dglucopyranoside (7)

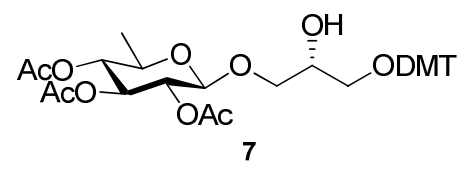


A solution of compound $18(225 \mathrm{mg}, 0.68 \mathrm{mmol})$ in dry pyridine- $\mathrm{CH}_{2} \mathrm{Cl}_{2}(1: 1,20 \mathrm{ml})$ was reacted following the general procedure for hydroxyl protection. The crude was purified by silica gel column chromathography (Hex/EtOAc, from 2:1 to 1:2) to give 7 (223 $\mathrm{mg}, 54 \%$ ) as a syrup. ${ }^{1} \mathrm{H}$ NMR (400 MHz, $\mathrm{CDCl}_{3}$ ) $\delta$ (ppm): 7.44 (d, 2H, $J=8.8 \mathrm{~Hz}, \mathrm{H}_{\text {arom}}$ ), 7.35-7.20 (m, 7H, $\mathrm{H}_{\text {arom }}$ ), 6.83 (d, $4 \mathrm{H}, J=8.8 \mathrm{~Hz}, \mathrm{H}_{\text {arom }}$ ), 5.17 (t, $\left.1 \mathrm{H}, J=9.6 \mathrm{~Hz}, \mathrm{H}_{3}\right), 4.97$ (dd, $1 \mathrm{H}, J=8.0 / 9.6 \mathrm{~Hz}$, $\left.\mathrm{H}_{2}\right), 4.86\left(\mathrm{t}, 1 \mathrm{H}, J=9.6 \mathrm{~Hz}, \mathrm{H}_{4}\right), 4.53\left(\mathrm{~d}, 1 \mathrm{H}, J=8.0 \mathrm{~Hz}, \mathrm{H}_{1}\right), 3.94-3.92(\mathrm{~m}, 1 \mathrm{H},-\mathrm{CH}-), 3.88-3.74$ $\left(\mathrm{m}, 8 \mathrm{H},-\mathrm{OCH}_{2^{-}}, 2 \mathrm{x}-\mathrm{OCH}_{3}\right), 3.62-3.58\left(\mathrm{~m}, 1 \mathrm{H}, \mathrm{H}_{5}\right), 3.24-3.16\left(\mathrm{~m}, 2 \mathrm{H},-\mathrm{OCH}_{2}\right), 2.05,2.01,1.97$ $\left(3 \mathrm{~s}, 6 \mathrm{H}, 3 \mathrm{x}-\mathrm{OCOCH}_{3}\right), 1.25\left(\mathrm{~d}, 3 \mathrm{H}, J=6.2 \mathrm{~Hz},-\mathrm{CH}_{3}\right) ;{ }^{13} \mathrm{C} \mathrm{NMR}\left(125 \mathrm{MHz}, \mathrm{CDCl}_{3}\right) \delta(\mathrm{ppm})$ : 170.3, 169.6, 169.4, 158.5, 144.8, 135.9, 130.5, 128.1, 127.8, 126.8, 113.1, 100.9, 86.1, 73.2, 72.8, 72.5, 71.7, 70.1, 69.8, 64.2, 55.2, 30.9, 20.7, 20.6, 17.3. HRMS (FAB ${ }^{+}$) Calcd. for $\mathrm{C}_{36} \mathrm{H}_{42} \mathrm{NaO}_{12}(\mathrm{M}+\mathrm{Na})$ : 689.2574, found; 689.2587 .

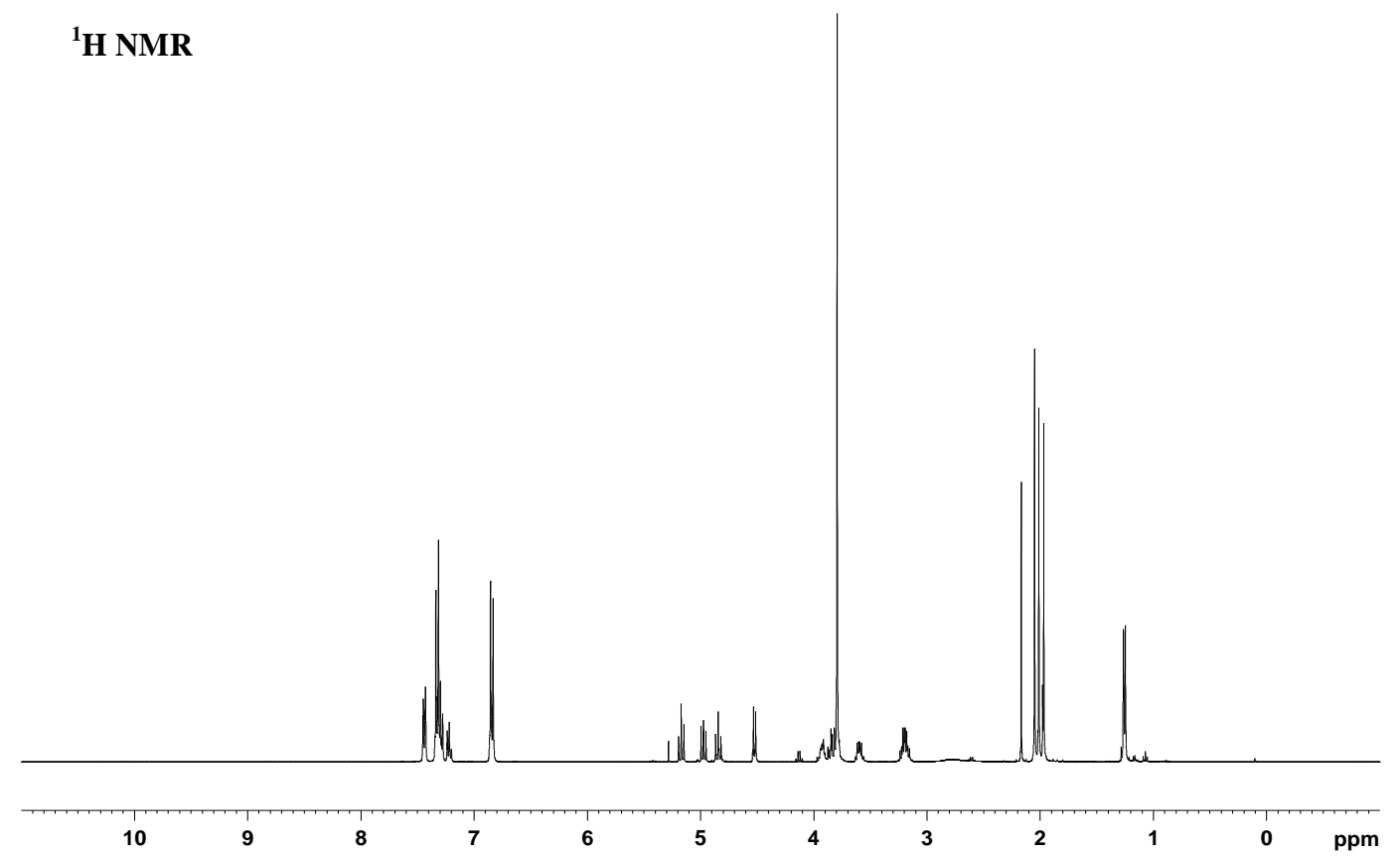




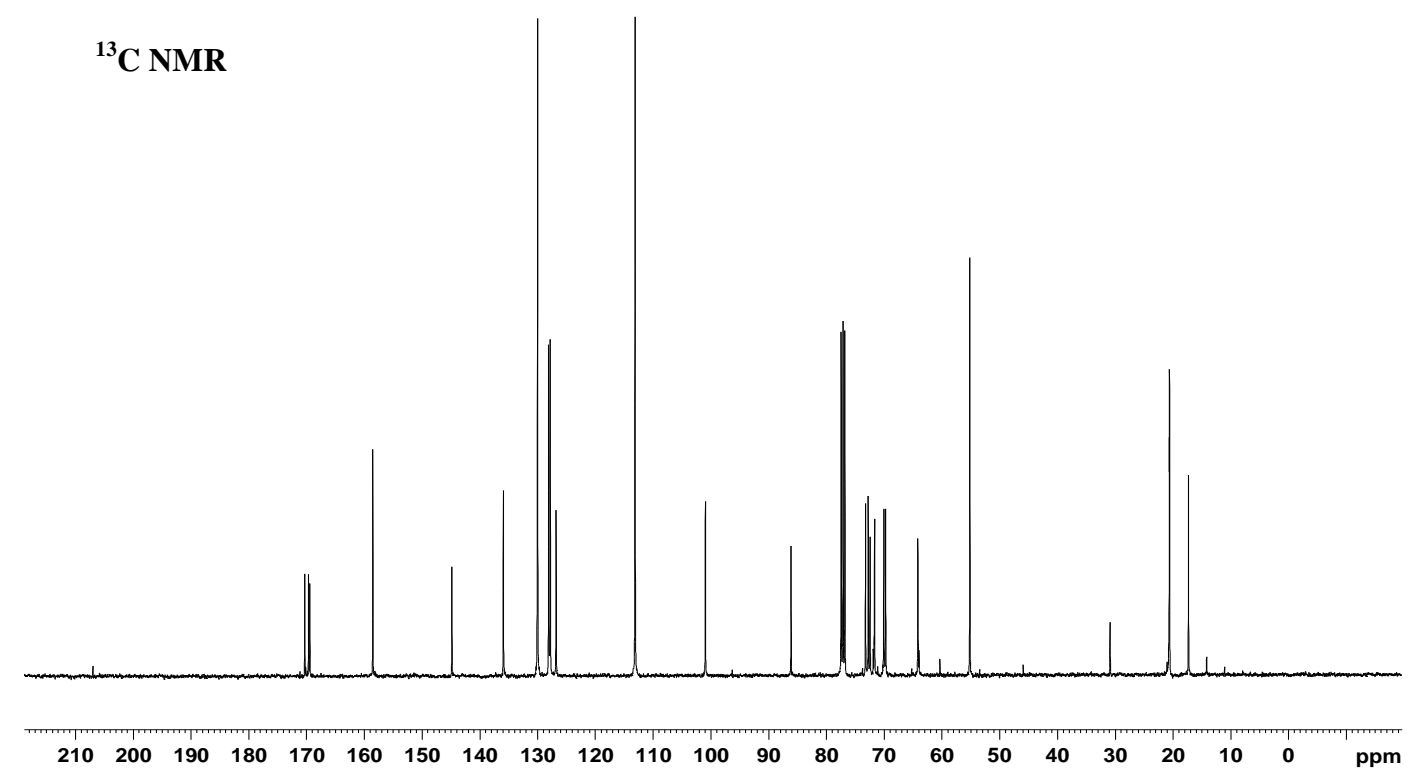

(S)-1-(4-(4,4'-dimethoxytrityloxy)-3-hydroxybutyl)thymine (15)

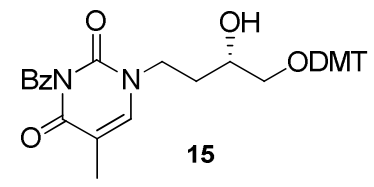

Compound $\mathbf{1 4}$ was reacted following the general procedure for hydroxyl protection. The reaction mixture was purified by flash chromatography (Hex/EtOAc, 1:2) to give compound $\mathbf{1 5}$ (745 mg, 87\%) as a white solid. ${ }^{1} \mathrm{H}$ NMR (400 MHz, $\left.\mathrm{CD}_{3} \mathrm{OD}\right) \delta$ (ppm): 7.93 (d, 2H, $J=7.6 \mathrm{~Hz}$, $\left.\mathrm{H}_{\text {benzoyl }}\right), 7.68\left(\mathrm{t}, 1 \mathrm{H}, J=7.6 \mathrm{~Hz}, \mathrm{H}_{\text {benzoyl }}\right), 7.53-7.7 .45\left(\mathrm{~m}, 5 \mathrm{H}, \mathrm{H}_{\text {benzoyl }} \mathrm{H}_{\text {thymine }}, \mathrm{H}_{\text {arom }}\right), 7.34(\mathrm{~d}, 4 \mathrm{H}$, $\left.J=8 \mathrm{~Hz}, \mathrm{H}_{\text {arom }}\right), 7.29-7.25\left(\mathrm{~m}, 3 \mathrm{H}, \mathrm{H}_{\text {arom }}\right), 7.21-7.17\left(\mathrm{~m}, 1 \mathrm{H}, \mathrm{H}_{\text {arom }}\right) 6.84\left(\mathrm{~d}, J=8 \mathrm{~Hz}, 4 \mathrm{H}, \mathrm{H}_{\text {arom }}\right)$, 3.95-3.80 (m, $\left.2 \mathrm{H},-\mathrm{CH}_{2}-\mathrm{CH}_{2} \mathrm{~N}-\right), 3.75\left(\mathrm{~s}, 6 \mathrm{H}, 2 \mathrm{x}-\mathrm{OCH}_{3}\right), 3.70-3.67\left(\mathrm{~m}, 1 \mathrm{H},-\mathrm{OCH}_{2} \mathrm{CH}(\mathrm{OH}) \mathrm{CH}_{2}-\right.$ ), 3.20-3.17 (m, 1H, - $\left.\mathrm{OCH} H \mathrm{CH}(\mathrm{OH}) \mathrm{CH}_{2}-\right), 3.06-3.02\left(\mathrm{~m}, 1 \mathrm{H},-\mathrm{OCH} \mathrm{HCH}(\mathrm{OH}) \mathrm{CH}_{2}-\right), 2.06-1.98$ $\left(\mathrm{m}, 1 \mathrm{H},-\mathrm{CH}(\mathrm{OH}) \mathrm{CH} H \mathrm{CH}_{2} \mathrm{~N}-\right), 1.91$ (s, 3H,- $\left.\mathrm{CH}_{3 \text { thymine }}\right), 1.76-1.68\left(\mathrm{~m}, 1 \mathrm{H},-\mathrm{CH}(\mathrm{OH}) \mathrm{CHHCH}_{2} \mathrm{~N}-\right.$ ); ${ }^{13} \mathrm{C}$ NMR (101 MHz, $\left.\mathrm{CD}_{3} \mathrm{OD}\right) \delta$ (ppm): 169.1, 163.8, 158.7, 150.0, 145.1, 142.4, 136.0, 134.9, 131.6, 130.0, 129.9, 129.0, 128.0, 127.4, 126.4, 112.7, 109.6, 86.0, 67.6, 67.4, 54.3, 45.9, 32.4, 10.9. HRMS ( $\left.\mathrm{EI}^{+}\right)$Calcd. for $\mathrm{C}_{37} \mathrm{H}_{36} \mathrm{~N}_{2} \mathrm{O}_{7}$ : 620.2523, found; 620.2526 . 

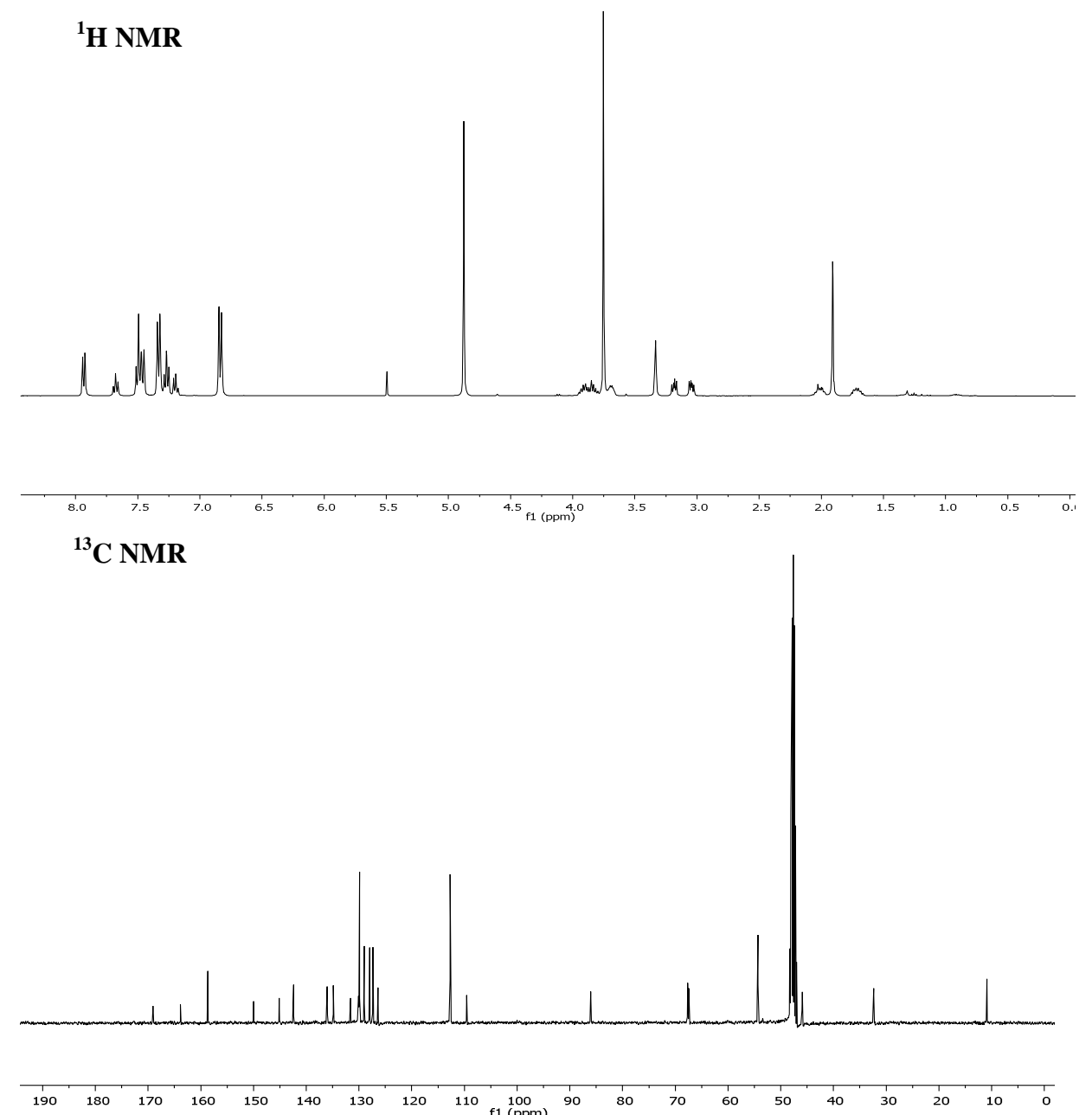

General procedure for synthesis of carbohydrate phosphoramidites. To a solution of DMT protected compound $(0.546 \mathrm{mmol})$ in dry $\mathrm{CH}_{2} \mathrm{Cl}_{2}(5 \mathrm{ml})$, DIPEA $(0.382 \mathrm{ml}, 2.185 \mathrm{mmol})$ and 2-cyanoethyl- $N, N^{\prime}$-diisopropylamino-chlorophosphoramidite 8 (0.183 $\left.\mathrm{ml}, 0.819 \mathrm{mmol}\right)$ were added at room temperature under argon atmosphere. After $1 \mathrm{~h}$ the solvent was evaporated to dryness. The product was purified by silica gel column chromathography using a mixture of hexane, ethyl acetate and triethylamine.

\section{2-(R)-1-(4,4'-dimethoxytrytiloxy)-3-(2,3,4,6-tetra- $O$-acetyl- $\beta$-D-glucopyranosyloxy)propyl} (2-cyanoethyl) ( $N, N^{\prime}$-diisopropyl) phosphoramidite (9)

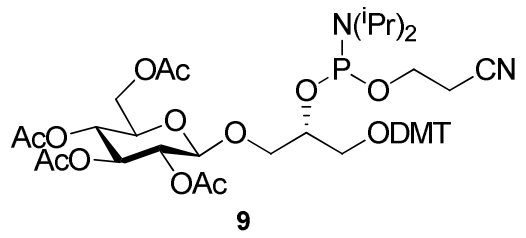


A solution of compound $6(236 \mathrm{mg}, 0.325 \mathrm{mmol})$ in anhydrous $\mathrm{CH}_{2} \mathrm{Cl}_{2}(5 \mathrm{ml})$ was reacted following the general procedure for phosphoramidite synthesis. The crude was purified by silica gel column chromatography (Hex/EtOAc, 3:2 with 5\% of $\mathrm{NEt}_{3}$ ) to give compound 9 (215 mg, 89\%) as a white solid. ${ }^{1} \mathrm{H}$ NMR $\left(400 \mathrm{MHz}, \mathrm{CDCl}_{3}\right) \delta$ (ppm) (mix of isomers): 7.437.13 (m, 9H, $\mathrm{H}_{\text {arom }}$ ), 6.85 (m, 4H, $\left.\mathrm{H}_{\text {arom }}\right)$, 5.18-4.85 (m, 3H, $\left.\mathrm{H}_{2}, \mathrm{H}_{3}, \mathrm{H}_{4}\right), 4.65-4.51\left(\mathrm{~m}, 1 \mathrm{H}, \mathrm{H}_{1}\right)$, 4.29-4.19 (m, 1H, -CH-), 4.29-3.44 (m, 15H, 2x- $\mathrm{OCH}_{2-},-\mathrm{OCH}_{2} \mathrm{CH}_{2} \mathrm{CN}, 2 \mathrm{x}-\mathrm{OCH}_{3}, \mathrm{H}_{5}, \mathrm{H}_{6}, \mathrm{H}_{6}$ ), 3.32-2.83 (m, 2H, 2xCH( $\left.\left.\mathrm{CH}_{3}\right)_{2}\right), 2.69-2.60\left(\mathrm{~m}, 2 \mathrm{H},-\mathrm{CH}_{2} \mathrm{CH}_{2} \mathrm{CN}\right), 2.17-1.69(\mathrm{~m}, 12 \mathrm{H}, 4 \mathrm{x}-$ $\left.\mathrm{OCOCH}_{3}\right), 1.32-1.00\left(\mathrm{~m}, 12 \mathrm{H}, 2 \mathrm{x}-\mathrm{CH}\left(\mathrm{CH}_{3}\right)_{2}\right) ;{ }^{13} \mathrm{C} \mathrm{NMR}\left(101 \mathrm{MHz}, \mathrm{CDCl}_{3}\right) \delta$ (ppm): 171.1, 170.7, 170.2, 169.3, 158.5 (2), 145.1, 136.8, 136.2, 130.4, 130.1, 130.0, 129.1, 128.35, 128.3, 127.8, 127.7, 126.9, 126.7, 118.0, 117.7, 116.2, 113.1, 100.7, 86.4, 76.8, 73.1, 72.6, 71.3, 68.4, $62.4,61.8,60.4,55.2,55.1,43.2,43.1,24.7,24.6,24.5,24.4,21.1,20.6,20.4,20.2 .{ }^{31} \mathrm{P}$ NMR $\left(162 \mathrm{MHz}, \mathrm{CDCl}_{3}\right) \delta(\mathrm{ppm}): 150.2$, 149.6. HRMS (FAB $\left.{ }^{+}\right)$Calcd. for $\mathrm{C}_{47} \mathrm{H}_{61} \mathrm{~N}_{2} \mathrm{KO}_{15} \mathrm{P}(\mathrm{M}+\mathrm{K})$ : 963.3447, found; 963.3597.

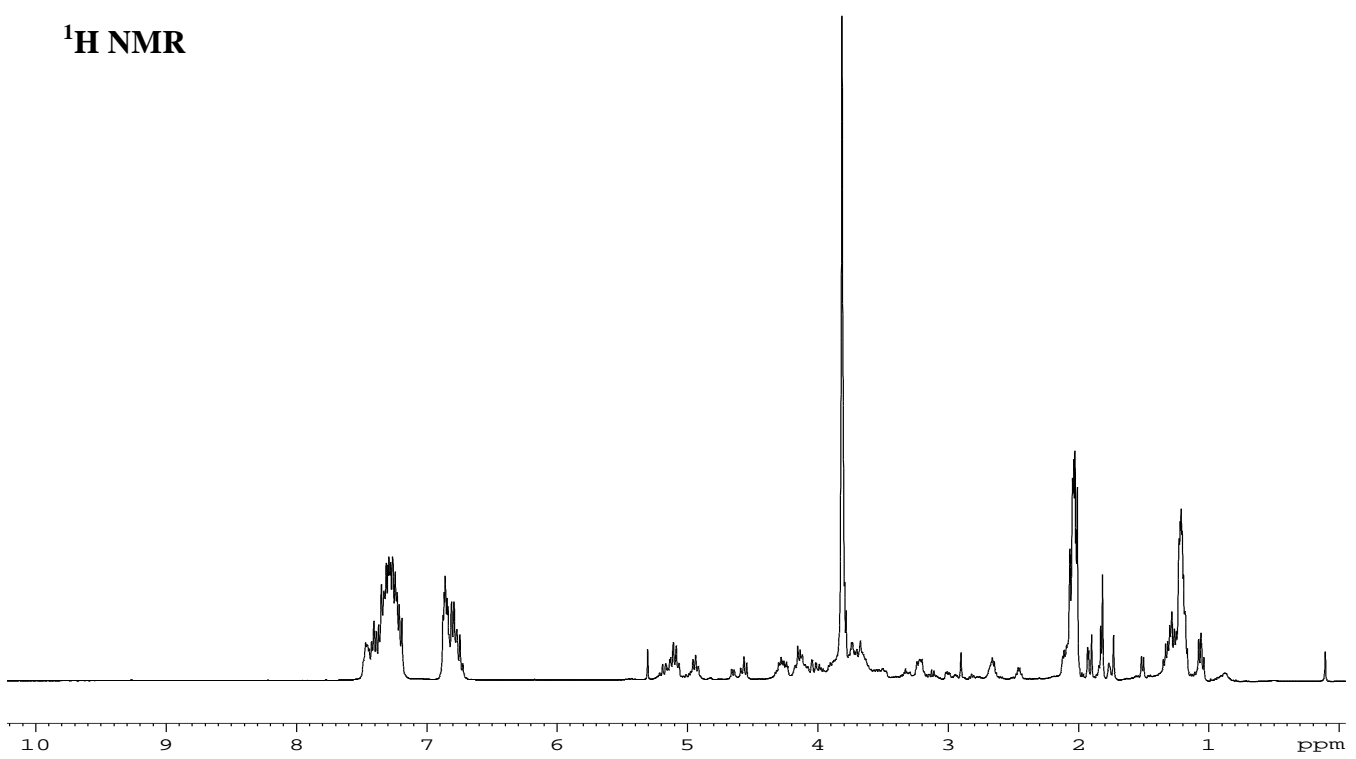




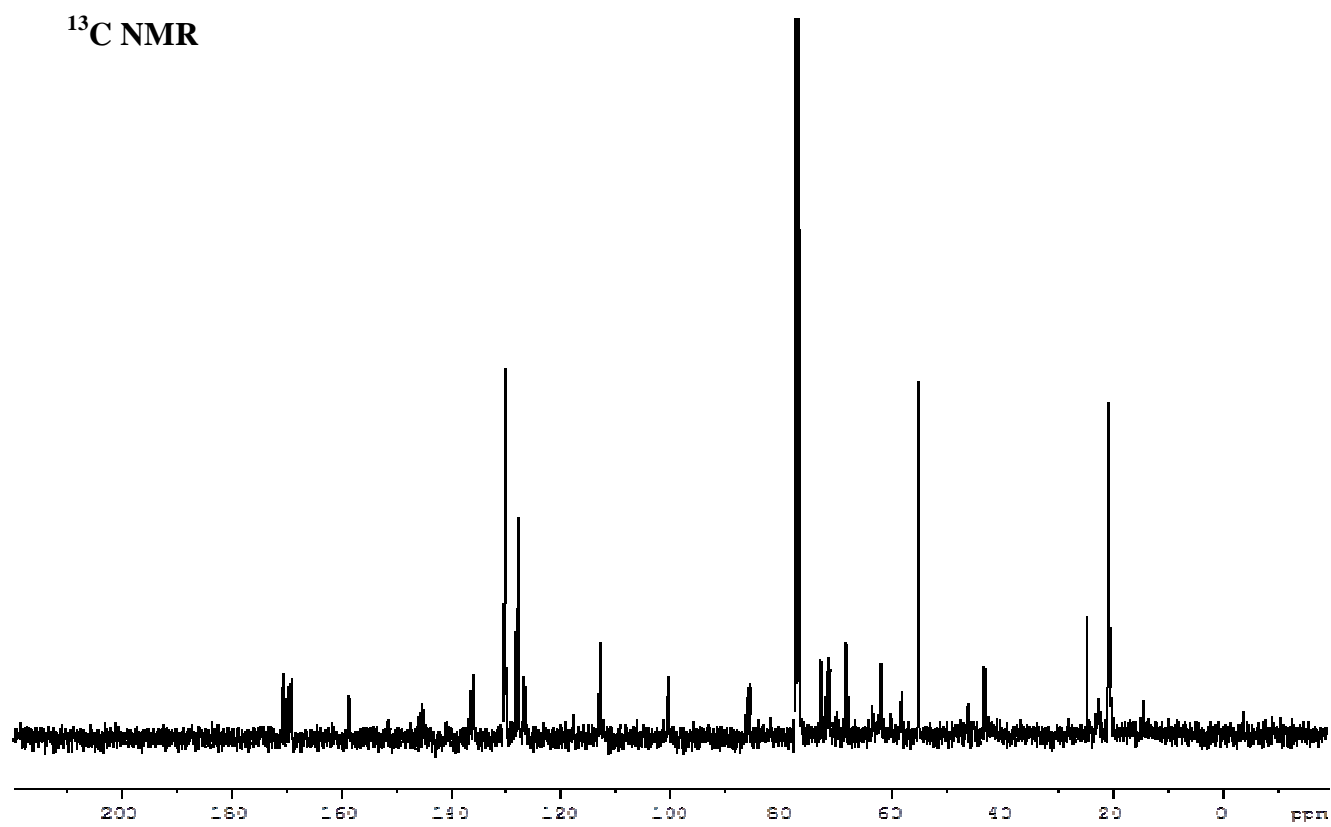

${ }^{31}$ P NMR

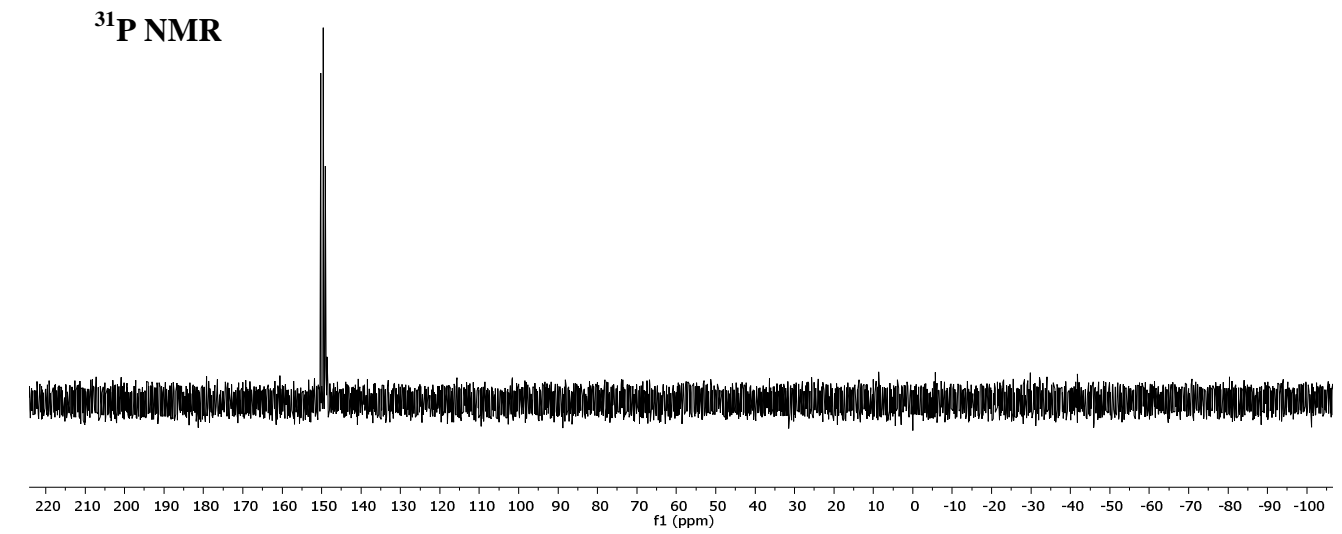

2-(R)-1-(4,4'-dimethoxytrytiloxy)-3-(2,3,4-tri- $O$-acetyl-6-deoxy- $\beta$-Dglucopyranosyloxy)propyl (2-cyanoethyl) $(N, N$ '-diisopropyl) phosphoramidite (10)

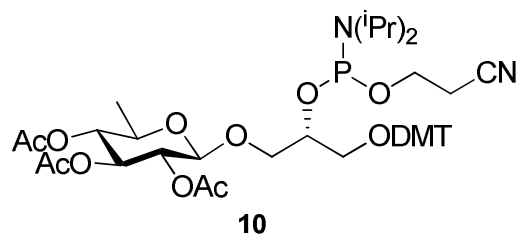

A solution of compound $7(200 \mathrm{mg}, 0.299 \mathrm{mmol})$ in anhydrous $\mathrm{CH}_{2} \mathrm{Cl}_{2}(5 \mathrm{ml})$ was reacted following the general procedure for phosphoramidite synthesis. The crude was purified by silica gel column chromatography (Hex/EtOAc, 2:1 with $5 \%$ of $\mathrm{NEt}_{3}$ ) to give compound $\mathbf{1 0}$ (230 mg, 89\%) as a glassy solid. ${ }^{1} \mathrm{H} \mathrm{NMR}\left(400 \mathrm{MHz}, \mathrm{CDCl}_{3}\right) \delta$ (ppm) (mix of isomers): 7.48$7.22\left(\mathrm{~m}, 9 \mathrm{H}, \mathrm{H}_{\text {arom }}\right), 6.85\left(\mathrm{t}, 4 \mathrm{H}, J=7.0 \mathrm{~Hz}, \mathrm{H}_{\text {arom }}\right), 5.16-5.08\left(\mathrm{~m}, 1 \mathrm{H}, \mathrm{H}_{3}\right), 4.94-4.78\left(\mathrm{~m}, 2 \mathrm{H}, \mathrm{H}_{4}\right.$, $\left.\mathrm{H}_{2}\right), 4.55\left(\mathrm{dd}, 1 \mathrm{H}, J_{\mathrm{H}, \mathrm{P}}=26.0 \mathrm{~Hz}, J=8.0 \mathrm{~Hz}, \mathrm{H}_{1}\right), 4.17-4.04(\mathrm{~m}, 1 \mathrm{H},-\mathrm{CH}-), 4.00-3.67(\mathrm{~m}, 10 \mathrm{H},-$ 
$\left.\mathrm{OCH}_{2-},-\mathrm{OCH}_{2} \mathrm{CH}_{2} \mathrm{CN}, 2 \mathrm{x}-\mathrm{OCH}_{3}\right), 3.66-3.47$ (m, 3H, 2x-CH( $\left.\left.\mathrm{CH}_{3}\right)_{2}, \mathrm{H}_{5}\right), 3.33-3.13(\mathrm{~m}, 2 \mathrm{H},-$ $\mathrm{OCH}_{2}$ ) $, 2.66-2.43\left(\mathrm{~m}, 2 \mathrm{H},-\mathrm{CH}_{2} \mathrm{CH}_{2} \mathrm{CN}\right), 2.05,2.00,1.92\left(3 \mathrm{~s}, 9 \mathrm{H}, 3 \mathrm{x}-\mathrm{OCOCH}_{3}\right), 1.29-1.16$ (m, $\left.12 \mathrm{H}, 2 \mathrm{x}-\mathrm{CH}\left(\mathrm{CH}_{3}\right)_{2}\right), 1.05\left(\mathrm{~d}, 3 \mathrm{H}, J=6.8 \mathrm{~Hz},-\mathrm{CH}_{3}\right) ;{ }^{13} \mathrm{C} \mathrm{NMR}\left(101 \mathrm{MHz}, \mathrm{CDCl}_{3}\right) \delta(\mathrm{ppm})$ : $170.3,169.7,169.3,169.2,158.5,149.9,136.1,130.1,128.2$, 128.1, 127.8, 126.8, 117.8, 113.1, 100.5, 86.1, 73.4, 73.0, 72.6, 72.4, 71.6, 70.2, 69.9, 69.5, 63.7, 58.4, 43.2, 24.7, 24.6, 24.5, 20.7, 20.6, 20.5, 17.3. ${ }^{31} \mathrm{P}$ NMR (162 MHz, $\left.\mathrm{CDCl}_{3}\right) \delta(\mathrm{ppm}):$ 149.5, 148.9.HRMS (FAB ${ }^{+}$) Calcd. for $\mathrm{C}_{45} \mathrm{H}_{59} \mathrm{~N}_{2} \mathrm{NaO}_{13} \mathrm{P}(\mathrm{M}+\mathrm{Na}): 889.3652$, found: 889.3683.

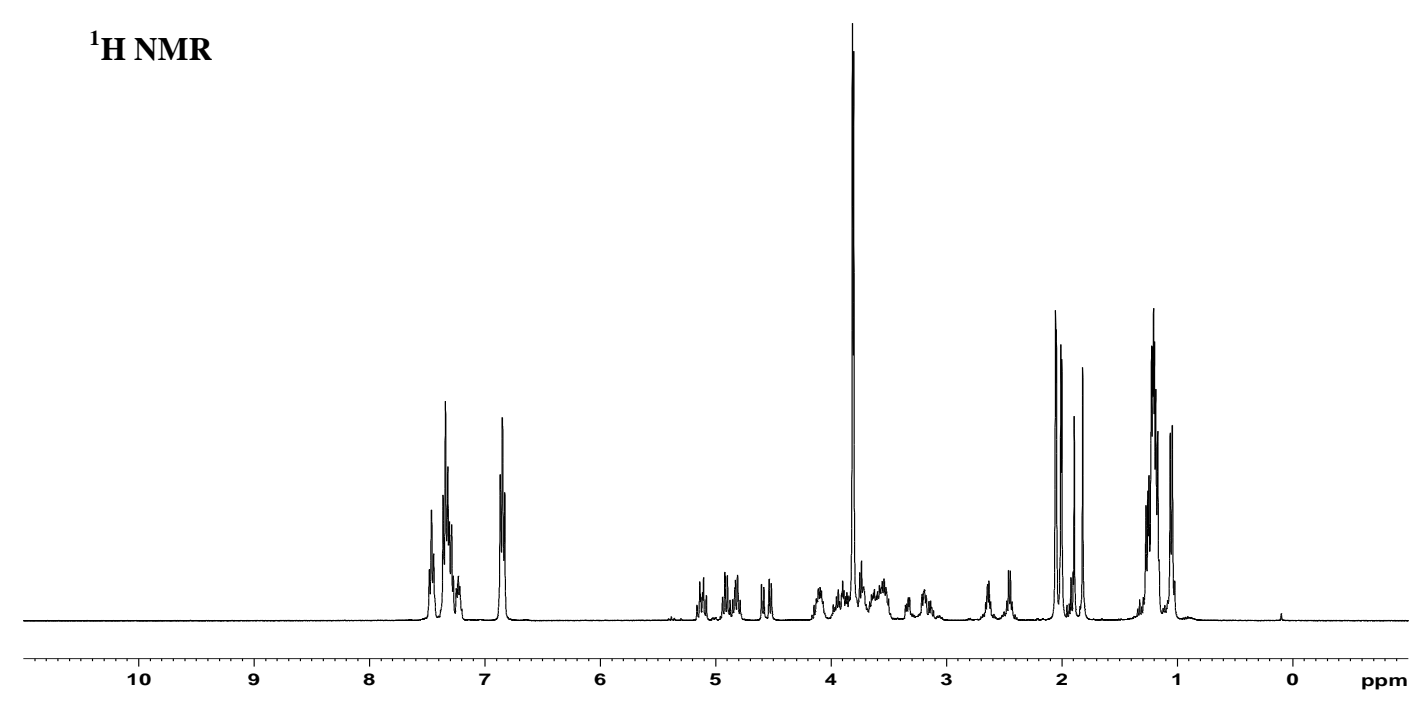

${ }^{13}$ C NMR

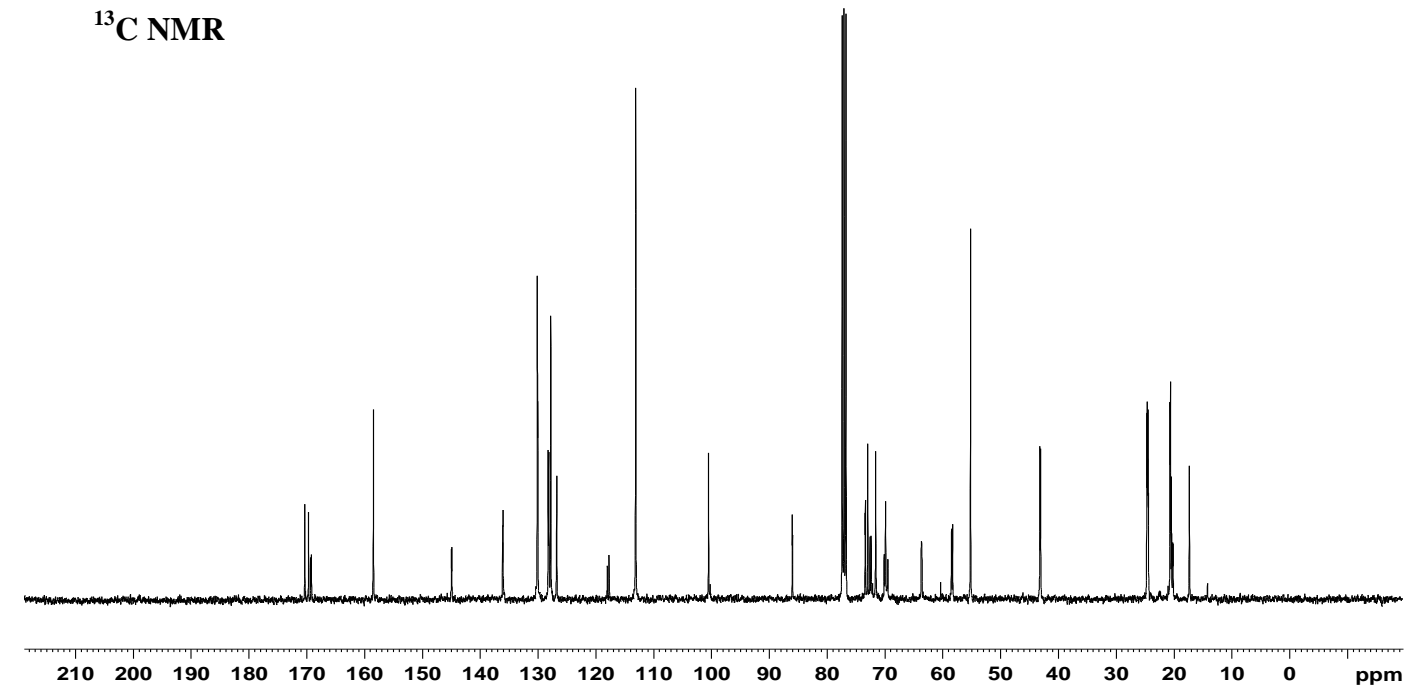




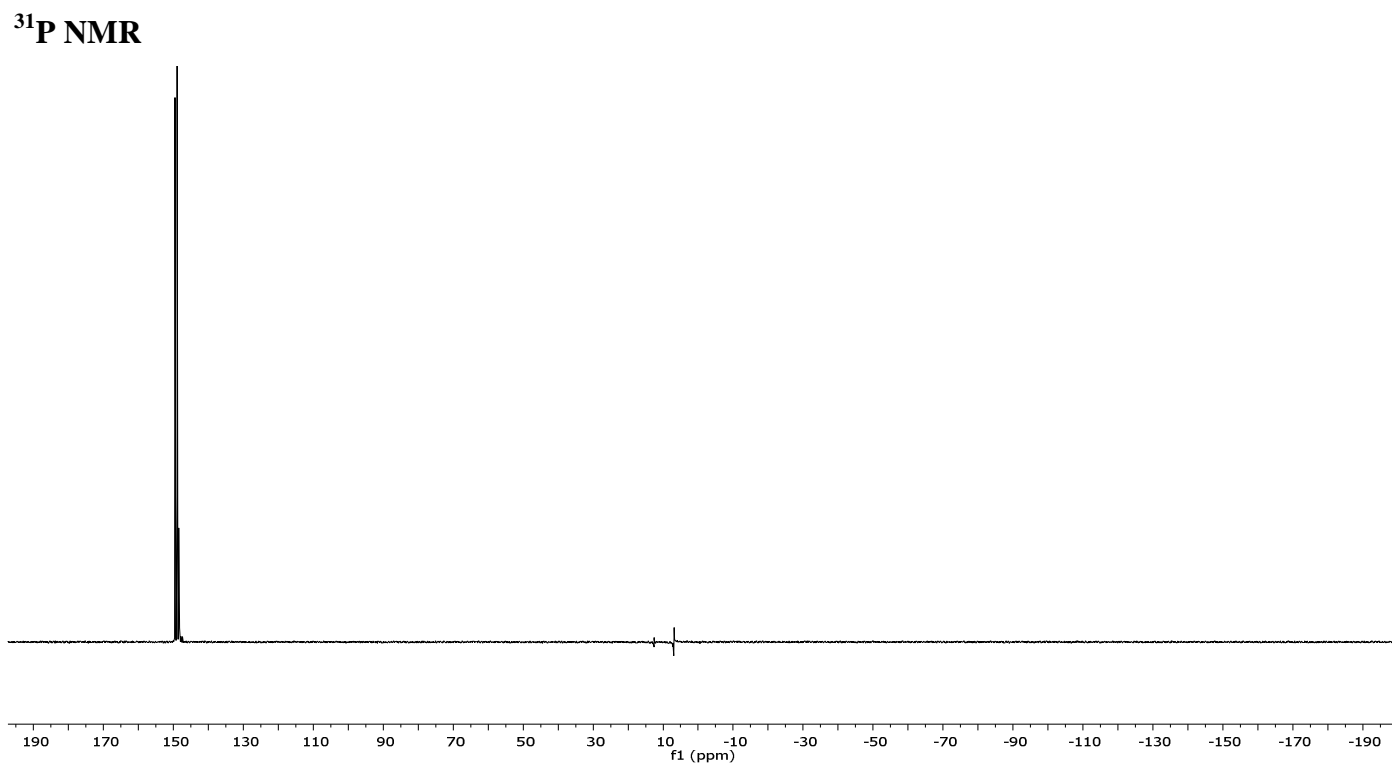

2-(S)-1-(4,4'-dimethoxytrytiloxy)-4-(thyminyl)butyl (2-cyanoethyl) $\quad\left(N\right.$ - $N^{\prime}$-diisopropyl) phosphoramidite (16)

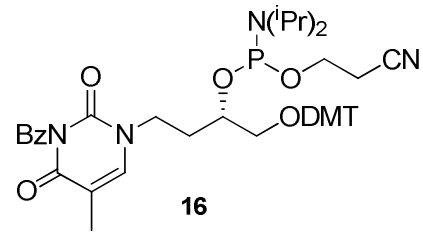

A solution of compound $15(339 \mathrm{mg}, 0.546 \mathrm{mmol})$ in anhydrous $\mathrm{CH}_{2} \mathrm{Cl}_{2}(5 \mathrm{ml})$ was reacted following the general procedure for phosphoramidite synthesis. The crude was purified by silica gel column chromatography (Hex/EtOAc, from 1:1 to $1: 3$, with $5 \%$ of $\mathrm{NEt}_{3}$ ) to give compound 16 as a white solid (360 mg, 80\%). ${ }^{1} \mathrm{H}$ NMR (400 MHz, $\left.\mathrm{CD}_{3} \mathrm{OD}\right) \delta(\mathrm{ppm})(\mathrm{mix}$ of isomers): 7.94 (d, 2H, $\left.J=8 \mathrm{~Hz}, \mathrm{H}_{\text {benzoyl }}\right), 7.70$ (t, $\left.1 \mathrm{H}, J=7.6 \mathrm{~Hz}, \mathrm{H}_{\text {benzoyl }}\right), 7.53-7.7 .46$ (m, 5H, $\left.\mathrm{H}_{\text {benzoyl }}, \mathrm{H}_{\text {thymine }}, 2 \mathrm{xH}_{\text {arom }}\right), 7.34\left(\mathrm{~d}, 4 \mathrm{H}, J=8.4 \mathrm{~Hz}, \mathrm{H}_{\text {arom }}\right), 7.30-7.25\left(\mathrm{~m}, 2 \mathrm{H}, \mathrm{H}_{\text {arom }}\right), 7.23-7.19$ (m, $\left.1 \mathrm{H}, \mathrm{H}_{\text {arom }}\right), 6.84$ (m, 4H, $\mathrm{H}_{\text {arom }}$ ), 4.14-4.02 (m, 1H, -CH-), 3.98-3.81 (m, 2H,- $\left.\mathrm{OCH}_{2}-\right), 3.76,3.75$ $\left(2 \mathrm{~s}, 6 \mathrm{H}, 2 \mathrm{x}-\mathrm{OCH}_{3}\right), 3.70-3.57\left(\mathrm{~m}, 3 \mathrm{H},-\mathrm{CH}_{2} \mathrm{O}-,-\mathrm{CH} H \mathrm{O}-\right), 3.35-3.30$ (m, 1H, -CHHO- ), 3.23$3.02\left(\mathrm{~m}, 1 \mathrm{H},-\mathrm{CH}\left(\mathrm{CH}_{3}\right)_{2}\right), 2.72-2.67\left(\mathrm{~m}, 1 \mathrm{H},-\mathrm{CH}\left(\mathrm{CH}_{3}\right)_{2}\right), 2.52-2.49\left(\mathrm{~m}, 1 \mathrm{H},-\mathrm{CH} H \mathrm{CH}{ }_{2} \mathrm{~N}-\right)$, 2.23-2.08 (m, 1H, - $\left.\mathrm{CHHCH}_{2} \mathrm{~N}-\right), 1.88\left(\mathrm{~s}, 3 \mathrm{H},-\mathrm{CH}_{3 \text { thymine }}\right), 1.35-1.10\left(\mathrm{~m}, 14 \mathrm{H},-\mathrm{CH}_{2} \mathrm{CN}, 4 \mathrm{x}-\right.$ $\left.\mathrm{CH}_{3 \text { isopropyl }}\right) .{ }^{13} \mathrm{C}$ NMR (101 MHz, $\left.\mathrm{CD}_{3} \mathrm{OD}\right) \delta(\mathrm{ppm}): 169.0,163.8,163.7,158.7,149.9,145.1$, 142.2 , 142.0, 135.9, 135.9, 135.8, 134.9, 131.6, 130.1, 130.0, 129.9 (2), 129.0, 128.0, 127.9, 127.4, 126.4, 118.4, 118.1, 112.7, 109.8, 109.6, 86.1, 71.4, 71.2, 71.1, 70.9, 66.0, 65.7, 58.3, 58.2, 58.1, 58.0, 54.3, 47.6, 47.4, 47.2, 47.0, 45.6, 45.5, 43.0, 42.9, 32.4, 32.1, 23.9, 23.8 (2), 23.7, 19.6, 19.5, 11.0. ${ }^{31} \mathrm{P}$ NMR (162 MHz, CD 3 OD) $\delta$ (ppm): 148.6, 148.1. HRMS (ES ${ }^{+}$Calcd. for $\mathrm{C}_{46} \mathrm{H}_{53} \mathrm{~N}_{4} \mathrm{NaO}_{7} \mathrm{P}(\mathrm{M}+\mathrm{Na})$ : 843.3493, found; 843.3488. 


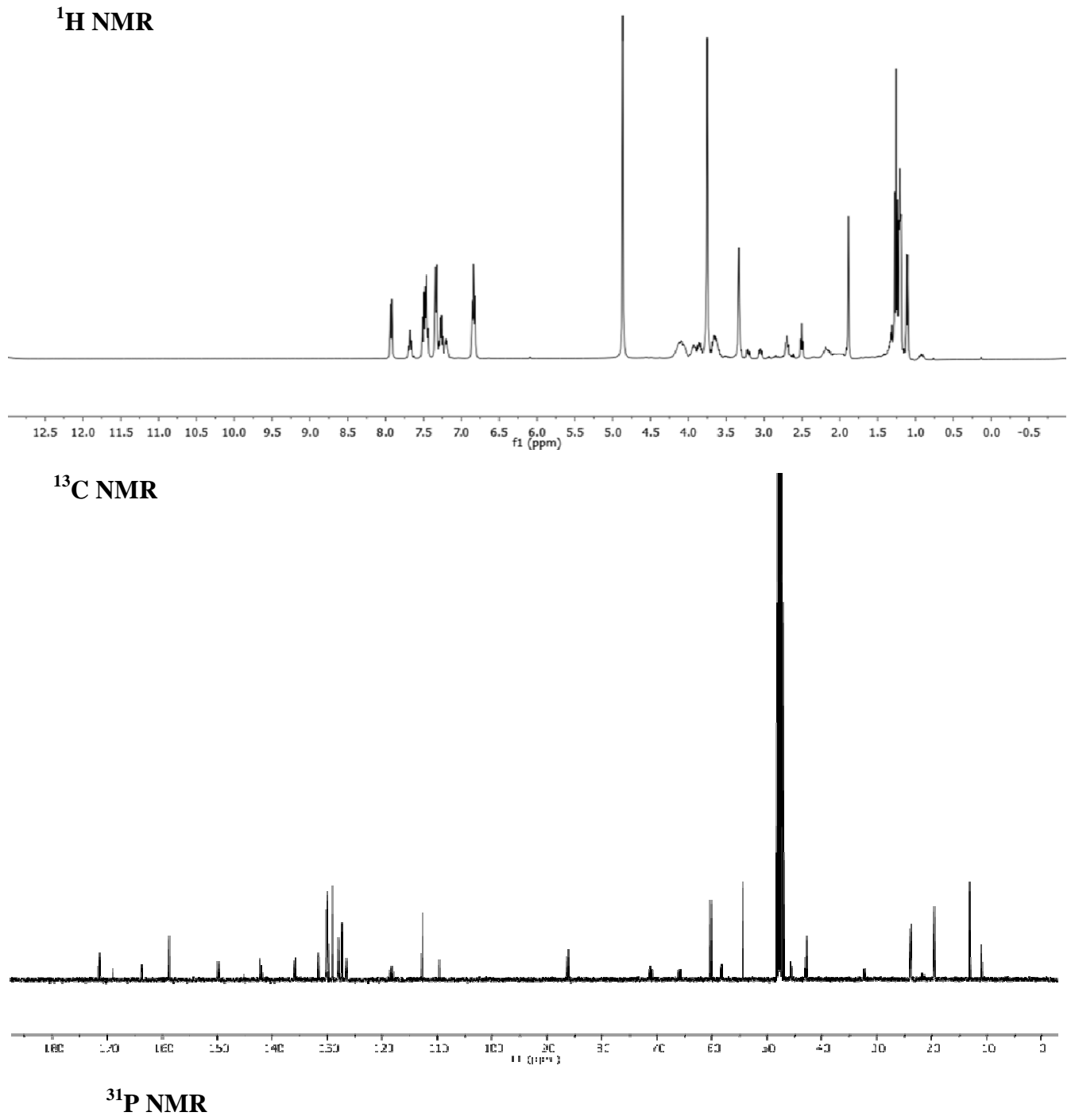




\section{Synthesis of natural and modified oligonucleotide DNA strands}

All natural and modified oligonucleotide DNA strands were synthesized by Biomers following standard $\beta$-cyanoethylphosphoramidite chemistry on $200 \mathrm{nmol}$ or $1 \mathrm{umol}$ scale and using the DMT-off procedure. Oligonucleotide supports were treated with 33\% aqueous ammonia for $16 \mathrm{~h}$ at $55^{\circ} \mathrm{C}$, then ammonia solutions were evaporated to dryness.

HPLC purification was carried out in a Waters Aliance 2690 RP-HPLC with a UV-Vis Photodiode Array (Waters) and using a Nucleosil 120 C18 (250 x 8 mm, $10 \mu \mathrm{m})$ column; 27 min linear gradient from 0 to $20 \% \mathrm{~B}$ (DMT off conditions); flow rate, $3 \mathrm{ml} / \mathrm{min}$; solution A was $5 \%$ acetonitrile in $0.1 \mathrm{M}$ aqueous triethylammonium acetate (TEAA, $\mathrm{pH}$ 6.5) buffer and solution B was $70 \%$ acetonitrile in $0.1 \mathrm{M}$ aqueous TEAA (pH 6.5). 
HPLC chromatograms of modified oligonucleotide DNA strands

5'-GATGACT*GCTAG

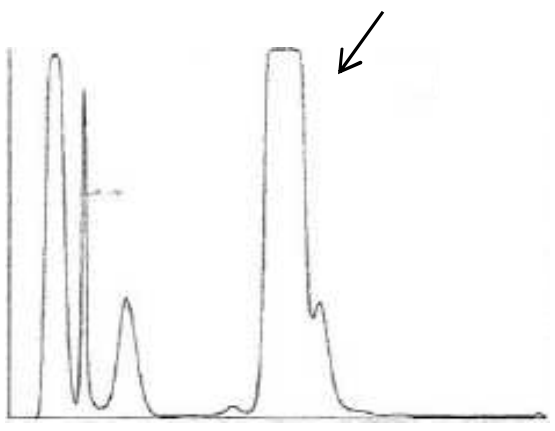

5'-GATGAC-6dglc-GCTAG

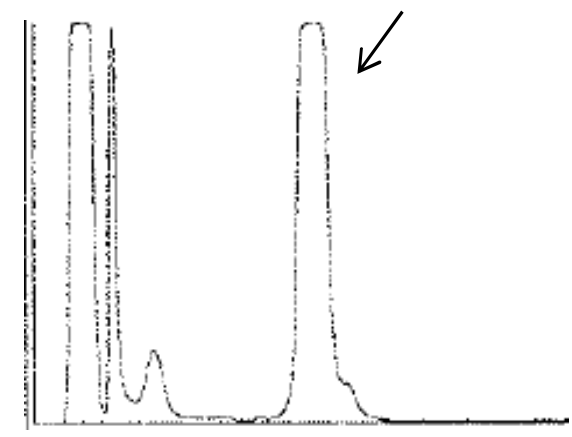

5'-CTAGC-T*-GTCATC

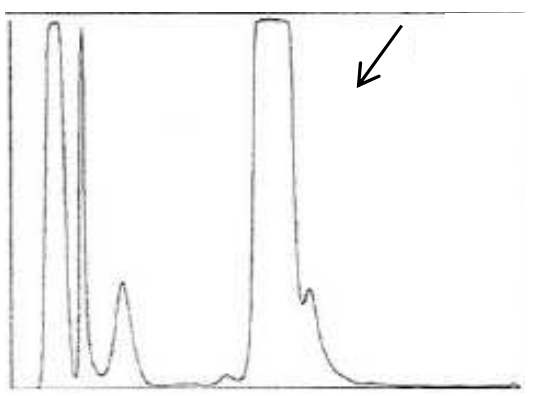

5'-GATGAC-glc-GCTAG

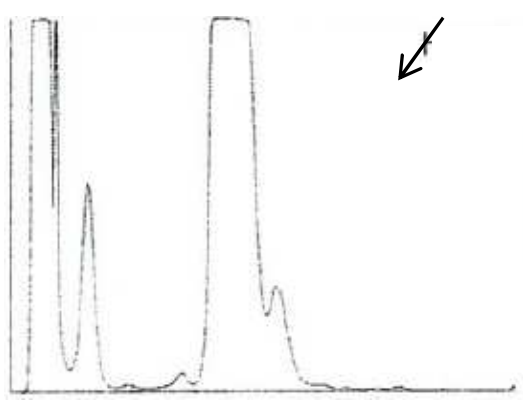

5'-CTAGC-glc-GTCATC

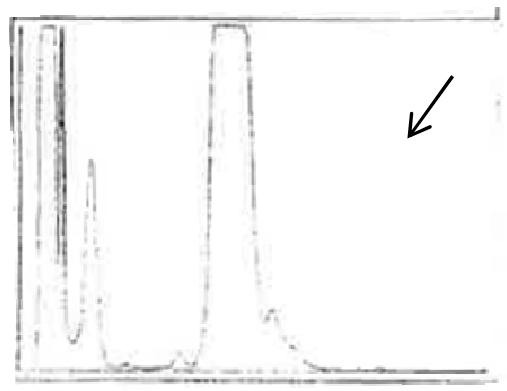


5'-CTAGC-6dglc-GTCATC

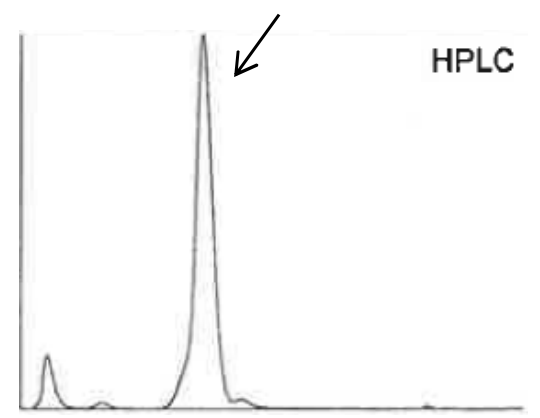

MALDI-TOF data of modified oligonucleotide DNA strands

\begin{tabular}{lcc}
\hline Carbohydrate oligonucleotide conjugates & $\begin{array}{c}{[\mathbf{M}-\mathbf{H}]} \\
\text { calc. }\end{array}$ & {$[\mathbf{M}-\mathbf{H}]$ exp. } \\
\hline $\begin{array}{l}\text { GATGAC-T*-GCTAG } \\
\text { (strand 1) }\end{array}$ & 3659 & 3661 \\
$\begin{array}{l}\text { GATGAC-glc-GCTAG } \\
\text { (strand 2) }\end{array}$ & 3698 & 3697 \\
$\begin{array}{l}\text { GATGAC-6dglc-GCTAG } \\
\text { (strand 3) }\end{array}$ & 3683 & 3681 \\
$\begin{array}{l}\text { CTAGC-T*-GTCATC } \\
\text { (strand 4) }\end{array}$ & 3570 & 3571 \\
$\begin{array}{l}\text { CTAGC-glc-GTCATC } \\
\text { (strand 5) }\end{array}$ & 3609 & 3606 \\
$\begin{array}{l}\text { CTAGC-6dglc-GTCATC } \\
\text { (strand 6) }\end{array}$ & 3594 & 3593 \\
\hline
\end{tabular}




\section{Synthesis of oligonucleotide GNA strands}

\section{a. Synthesis of GNA Phosphoramidites.}

The synthesis of DMT-phosphoramidite glycidol A and $\mathrm{T}$ followed the reported procedure described by Meggers et al. ${ }^{2}$

b. GNA Solid support synthesis. To $25 \mathrm{mg}(0.049 \mathrm{mmol})$ of DMT-glycidol $\mathrm{T}$ were added succinic anhydride $7.4 \mathrm{mg}(0.074 \mathrm{mmol})$ and $9.0 \mathrm{mg}(0.074 \mathrm{mmol})$ of dimethyaminopyridine in anhydrous DCM $(10 \mathrm{ml})$. The mixture was stirred overnight and then washed with $0.1 \mathrm{M}$ $\mathrm{NaH}_{2} \mathrm{PO}_{4}$ and dried over $\mathrm{MgSO}_{4}$. The solution was concentrated and the succinic derivative was used without further purification for the coupling to the solid support. We then followed the described procedure, ${ }^{2} 9.3 \mathrm{mg}$ (0.03 mmol) of 2,2'-dithio-bis-(5-nitropyridine) dissolved in 120 $\mu \mathrm{lACN}$ : dichloroethane (1:3) was mixed with a solution of the succinic derivative $20 \mathrm{mg}(0.03$ $\mathrm{mmol})$ and $3.66 \mathrm{mg}(0.03 \mathrm{mmol})$ DMAP dissolved in $500 \mu \mathrm{l}$ of ACN. $7.8 \mathrm{mg}(0.03 \mathrm{mmol})$ of $\mathrm{PPh}_{3}$ dissolved in $60 \mu \mathrm{l}$ of $\mathrm{ACN}$ were then added to clear the obtained solution. The mixture was vortexed and added to a vial containing $150 \mathrm{mg}(10.7 \mu \mathrm{mol})$ of LCAA-CPG $500 \AA$ for 4 hours at room temperature. Then, the resin was washed with ACN and DCM. The unreacted amino groups were acetylated with $1 \mathrm{ml}$ of two capping solutions (acetic anhydride/pyridine/ tetrahydrofuran (1:1:8) (capping A), 10\% $N$-methylimidazole in tetrahydrofurane (capping B). Trityl analysis yielded a loading of $18 \mu \mathrm{mol} / \mathrm{g}$.

c. GNA Oligonucleotide synthesis and purification. DNA oligonucleotides were synthesized on the $0.2 \mu \mathrm{mol}$ scale on an Applied Biosystems 394 synthesizer following standard conditions. Modified amidites were dissolved in anhydrous acetonitrile $(0.1 \mathrm{M})$ and all oligonucleotides were synthesized in DMT-ON mode. Then the solid support treated twice with $0.1 \mathrm{M}$ DBU/ACN for five minutes and washed with $1 \% \mathrm{Et}_{3} \mathrm{~N} / \mathrm{ACN}$. The solid support was transferred to a screw-cap glass vial and incubated at room temperature for four hours with $1.5 \mathrm{~mL}$ of $\mathrm{NH}_{3}$ solution (33\%).The ammonia solutions were concentrated to dryness and the product was desalted on NAP-10 (Sephadex G-25) columns eluted with water. All the oligonucleotides were purified by HPLC. Semipreparative column: X-bridge ${ }^{\mathrm{TM}}$ OST $\mathrm{C}_{18}(10 \times 50 \mathrm{~mm}, 2.5 \mu \mathrm{m}) ; 30 \mathrm{~min}$ linear gradient from $0 \%$ to $70 \%$, flow rate $2 \mathrm{~mL} / \mathrm{min}$; solution A was $5 \% \mathrm{ACN}$ in $0.1 \mathrm{M}$ aqueous TEAA and B 70\% ACN in $0.1 \mathrm{M}$ aqueous TEAA. The desired DMT-ON product corresponds to the main peak followed by secondary peak with similar retention time. The pure fractions were combined and evaporated to dryness. The residue that was obtained was treated with $200 \mu \mathrm{L}$ of $10 \% \mathrm{AcOH}$ solution and incubated at room temperature for $5 \mathrm{~min}$. The aqueous solution was neutralized with TEAA and, finally, the deprotected oligonucleotide was desalted 
in a NAP-10 column. All oligonucleotides were quantified by absorption at $260 \mathrm{~nm}$ and confirmed by MALDI mass spectrometry. Matrix-assisted laser desorption ionization time-offlight (MALDI-TOF) mass spectra were recorded on a Voyager-DETMRP spectrometer (Applied Biosystems) in negative mode (2,4,6- trihidroxyacetophenone matrix with ammonium citrate as an additive), (Servei d'Espectrometría de Masses, Universitat de Barcelona).

\section{HPLC chromatograms of oligonucleotide GNA strands}

3'- TAAAATTTATATTATTAA-2'

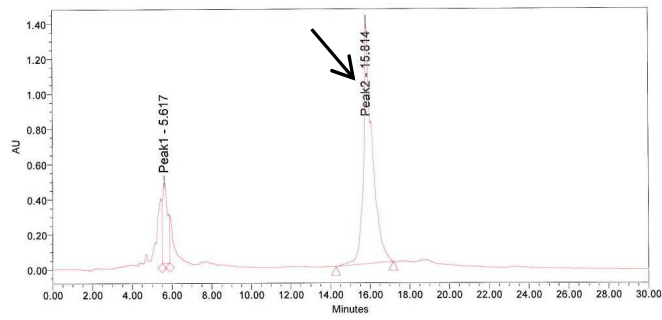

3'- TTAATAATTTAAATTTTA-2'

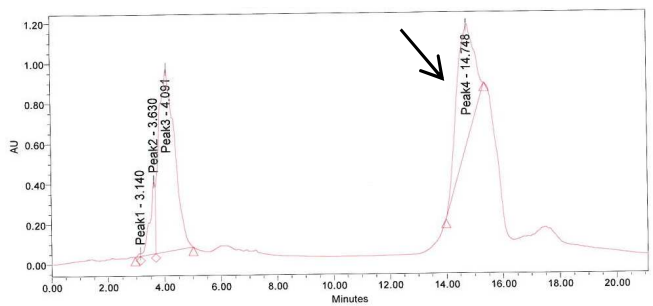

\section{3'- TTAATAAT-6dglc-TAAATTTTA-2'}

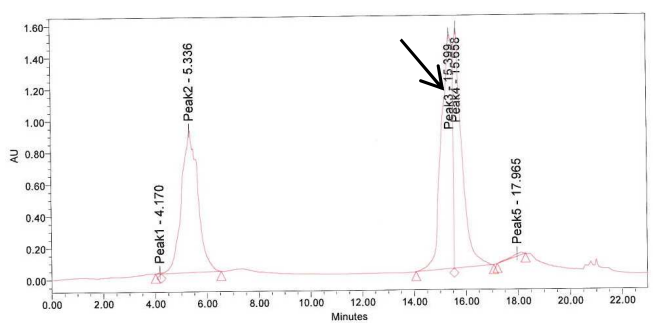

\section{3'- TTAATAATATAAATTTTA-2'}

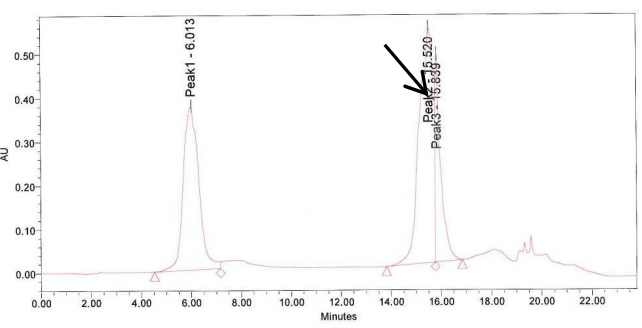

3'- TAAAATTTA-6dglc-ATTATTAA-2'

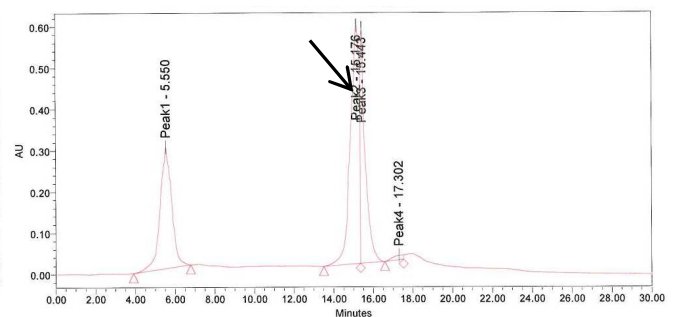

\section{MALDI-TOF data of oligonucleotide GNA strands}

\begin{tabular}{lll}
\hline GNA oligonucleotide sequence & [M-H] calc & [M-H] found \\
\hline 3'- TAAAATTTATATTATTAA-2' & 4735 & 4738 \\
3'- TTAATAATATAAATTTTA-2' & 4735 & 4737 \\
3'- TTAATAATTTAAATTTTA-2' & 4726 & 4726 \\
\hline
\end{tabular}




\begin{tabular}{lll}
\hline 3'- TAAAATTTA6dglcATTATTAA-2' & 4769 & 4773 \\
3'- TTAATAAT6dglcTAAATTTTA-2' & 4760 & 4764 \\
\hline
\end{tabular}




\section{Thermal denaturation methods.}

\section{DNA duplexes.}

UV-melting curves were measured on a Perkin-Elmer Lambda 750 UV/Vis spectrophotometer. Absorbance of duplexes in a 1:1 stoichiometric ratio were monitored at $260 \mathrm{~nm}$ and the heating rate was set to $1.0^{\circ} \mathrm{C} \cdot \mathrm{min}^{-1}$ from 10 to $80{ }^{\circ} \mathrm{C}$. The extinction coefficients of the natural oligonucleotide parts, $\varepsilon_{\text {nat }}$, was calculated using a oligo calculator (www.ambion.com). The total absorption coefficient was then calculated by simple addition: $\varepsilon=\varepsilon_{\text {nat1 }}+\varepsilon_{\text {nat2 }}$. The first derivative of the melting curves was obtained using Origin 8.0 software. To avoid air water condensation samples were measured in a nitrogen atmosphere.

\section{GNA duplexes.}

The absorbance versus temperature curves of duplexes were measured at $0.7 \mu \mathrm{M}$ strand concentration in $10 \mathrm{mM}$ sodium phosphate buffer at $\mathrm{pH} 7$ implemented with $200 \mathrm{mM} \mathrm{NaCl}$. Thermal UV experiments were performed in Teflon-stopped $1 \mathrm{~cm}$ path length quartz cells on a JACSO V-650 spectrophotometer equipped with thermoprogrammer. The samples were heated to $90^{\circ} \mathrm{C}$, allowed to slowly cool down to $20^{\circ} \mathrm{C}$, and then warmed during the denaturation experiments at a rate of $0.5^{\circ} \mathrm{C} / \mathrm{min}$ from 10 to $85^{\circ} \mathrm{C}$, monitoring absorbance at $260 \mathrm{~nm}$. Melting temperatures $\left(\mathrm{T}_{\mathrm{m}}\right)$ were determined by computerfit of the first derivative of absorbance with respect to $1 / \mathrm{T}$.

\section{NMR Spectroscopy.}

Samples of all the conjugates and control duplexes were suspended in $500 \mu \mathrm{L}$ of either $\mathrm{D}_{2} \mathrm{O}$ or $\mathrm{H}_{2} \mathrm{O} / \mathrm{D}_{2} \mathrm{O} 9: 1$ in phosphate buffer $10 \mathrm{mM}, 150 \mathrm{mM} \mathrm{NaCl}$, pH 7. NMR spectra were acquired in Bruker Avance spectrometers operating at 600 or $800 \mathrm{MHz}$ and were processed with Topspin software. DQF-COSY, TOCSY, and NOESY experiments were recorded in $\mathrm{D}_{2} \mathrm{O}$. The NOESY spectra were acquired with mixing times of 150 and $300 \mathrm{~ms}$, and the TOCSY spectra were recorded with standard MLEV-17 spin-lock sequence, and $80 \mathrm{~ms}$ mixing time. NOESY spectra in $\mathrm{H}_{2} \mathrm{O}$ were acquired with $100 \mathrm{~ms}$ mixing time. In $2 \mathrm{D}$ experiments in $\mathrm{H}_{2} \mathrm{O}$, water suppression was achieved by including a WATERGATE module in the pulse sequence prior to acquisition. Two-dimensional experiments in $\mathrm{D}_{2} \mathrm{O}$ were carried out at temperatures ranging from 5 to $25{ }^{\circ} \mathrm{C}$, whereas spectra in $\mathrm{H}_{2} \mathrm{O}$ were recorded at $5{ }^{\circ} \mathrm{C}$ to reduce the exchange with water. The spectral analysis program Sparky was used for semiautomatic assignment of the NOESY cross-peaks and quantitative evaluation of the NOE intensities. Distance constraints with their 
corresponding error bounds were incorporated into the AMBER potential energy by defining a flat-well potential term.

\section{Structure Calculations.}

Structures were calculated with the SANDER module of the molecular dynamics package AMBER. Starting models of the conjugate duplexes were built using the program SYBYL. The DNA moieties in the starting models were set to a standard B- canonical structure. These structures were taken as starting points for the AMBER refinement, which started with an annealing protocol in vacuo (using hexahydrated $\mathrm{Na}^{+}$counterions placed near the phosphates to neutralize the system). The resulting structures from in vacuo calculations were placed in the center of a water-box with around 4000 water molecules and 22 sodium counterions to obtain electroneutral systems. The structures were then refined including explicit solvent, periodic boundary conditions and the Particle-Mesh-Ewald method to evaluate long-range electrostatic interactions. Force field parameters for the carbohydrate moieties were taken from GLYCAM. The TIP3PBOX model was used to describe water molecules. The protocol for the constrained molecular dynamics refinement in solution consisted of an equilibration period of $160 \mathrm{ps}$ using a standard equilibration process, followed by four independent $500 \mathrm{ps}$ runs. Averaged structures were obtained by averaging the last $20 \mathrm{ps}$ of individual trajectories and further energy minimization of the structure. Analysis of the representative structures as well as the MD trajectories was carried out with the program MOLMOL and the analysis tools of AMBER.

\section{DFT quantum chemical methods.}

All calculations were performed with the Amsterdam density functional program (ADF 2013.1) (http://www.scm.com/)..$^{3-5}$ Calculations were initiated from the coordinates of the base pairs of interest in the PDB structures $(2 \mathrm{~N} 9 \mathrm{~F}$ and $2 \mathrm{~N} 9 \mathrm{H})$ we obtained previously from NMR studies and molecular dynamics. The original guanine and thymine bases in the obtained structures were changed to adenine and cytosine, respectively, in order to evaluate the four possible pairs with the natural DNA bases. Bases, base-base pairs, sugar-base pairs and sugarsugar pairs were optimized at the BLYP-D3(BJ)/TZ2P level of dispersion-corrected density functional theory (DFT) without any symmetrical constraints. Benchmarks have shown that dispersion-corrected DFT reproduces well high level ab initio results for weakly interacting systems (that is hydrogen bonding or stacking interactions). ${ }^{6}$ Solvent effects in aqueous solution are described with the COnductor-like Screening MOdel (COSMO), which takes effectively 
into account solute-solvent interactions, cavitation, internal energy and entropy effects of the solvent and yields an estimate of the Gibbs free energies. ${ }^{7-9}$ 
Figure S1. Synthesis of flexible T* amidite. N-benzyl-thymine 11 was used as starting material. ${ }^{1}$ Reagents and conditions: (i) $\mathrm{K}_{2} \mathrm{CO}_{3}$, TBAI, DMF; (ii) $\mathrm{AcOH}-\mathrm{H}_{2} \mathrm{O}, 80{ }^{\circ} \mathrm{C}$; (iii) DMTCl, pyridine, DMAP, $\mathrm{CH}_{2} \mathrm{Cl}_{2}$; (iv) DIPEA, $\mathrm{CH}_{2} \mathrm{Cl}_{2}$.

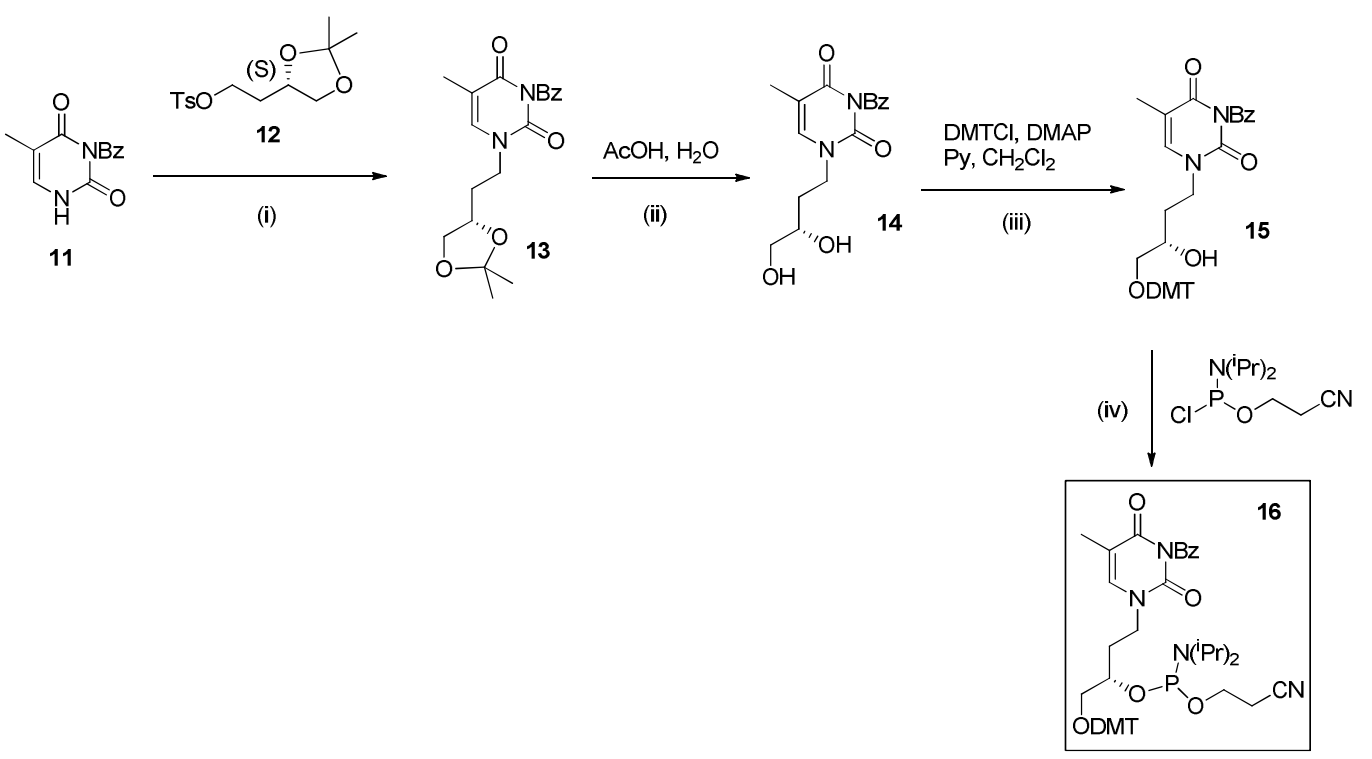


Figure S2: Melting curves for DNA duplexes containing glc-T, glc-G, 6dglc-C and 6dglc-A.

\section{5'-d(GATGACXGCTAG) \\ 3'-d(CTACTGYCGATC)}
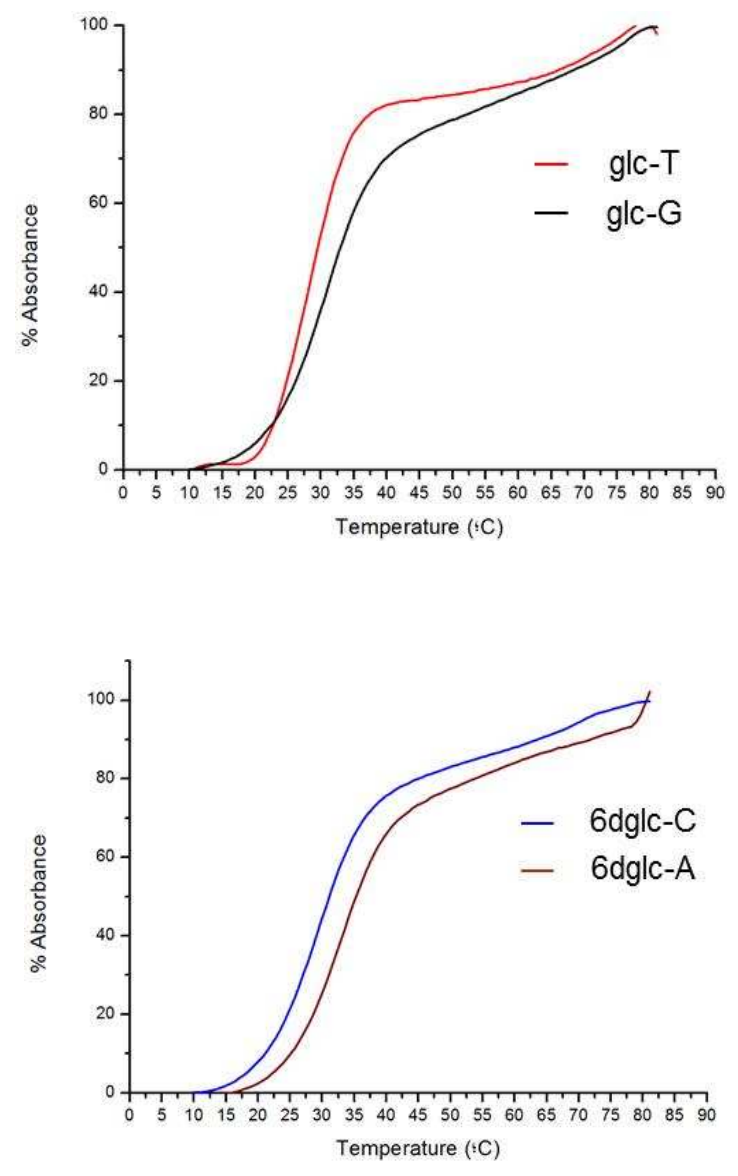
Figure S3: Melting curves for GNA double helices

\section{3'-TAAAATTTAXATTATTAA \\ 2'-ATTTTAAATYTAATAATT}
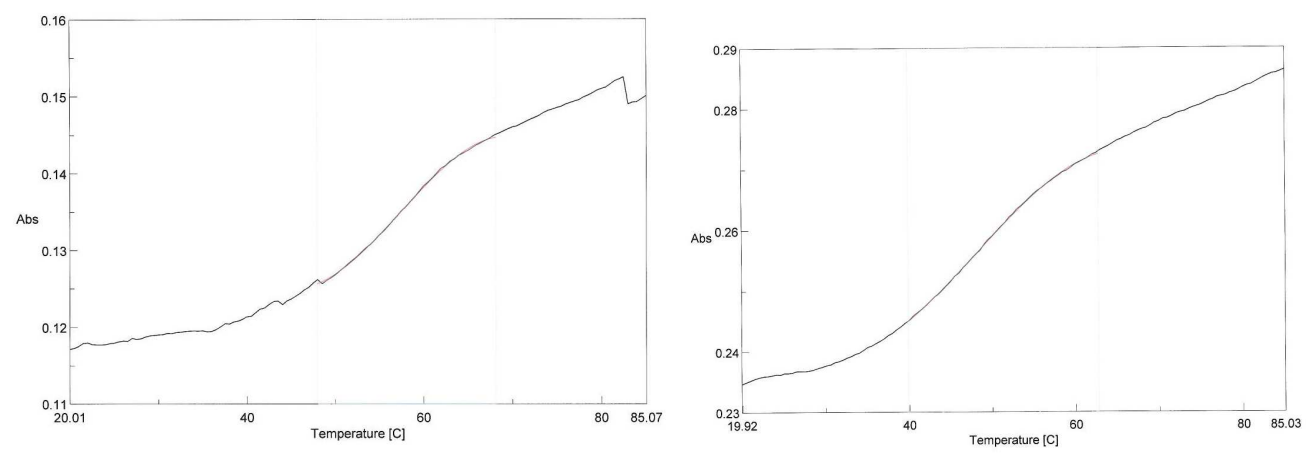

TA pair

\section{TT pair}

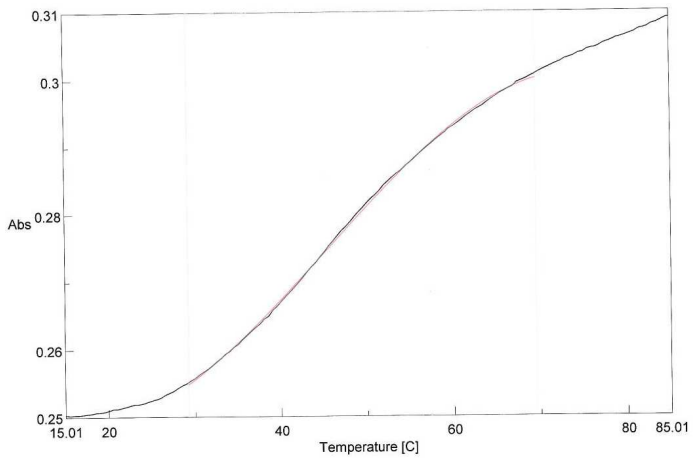

6dglc-A pair

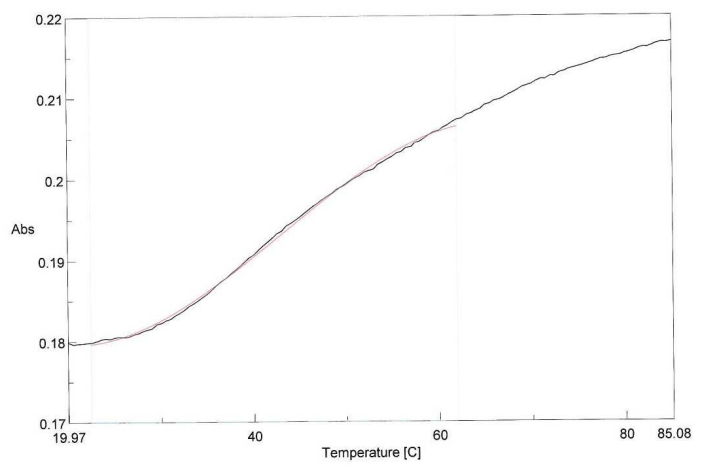

6dglc-6dglc pair

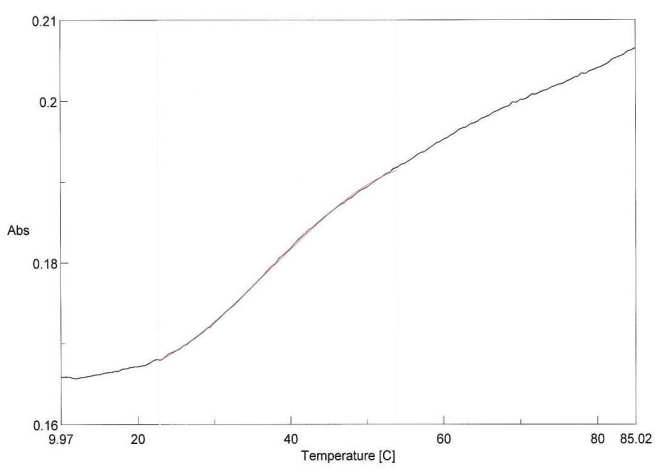

T-6dglc pair 
Figure S4. Imino region of the NMR spectra of helix 6dglc-G and helix 6dglc-T at $5{ }^{\circ} \mathrm{C}(10$ $\mathrm{mM}$ sodium phosphate, $150 \mathrm{mM} \mathrm{NaCl}, \mathrm{pH} 7$ ).
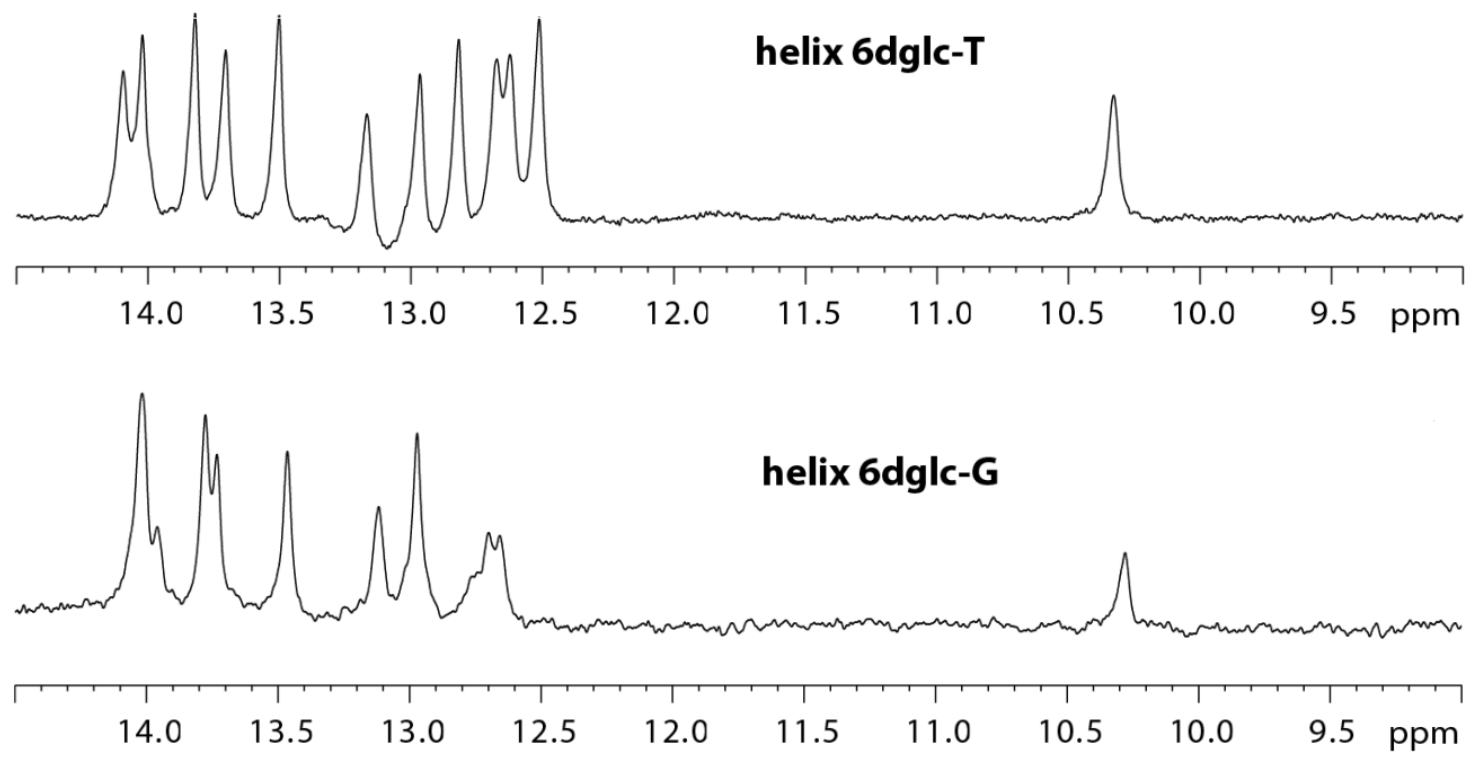

Figure S5.-More significant changes in proton chemical shifts along the sequence for helix 6dglc-G and helix 6dglc-T with respect to double helices containing all natural base pairs.

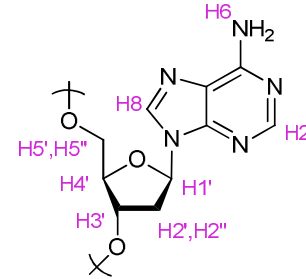

A

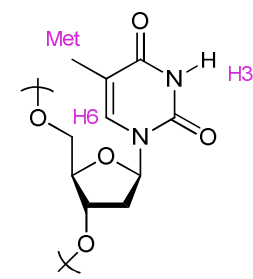

$\mathbf{T}$

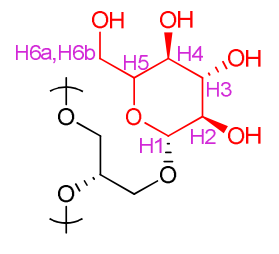

glc

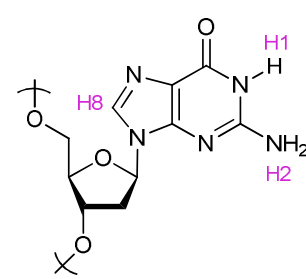

G

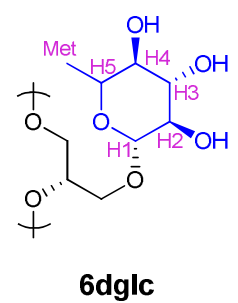




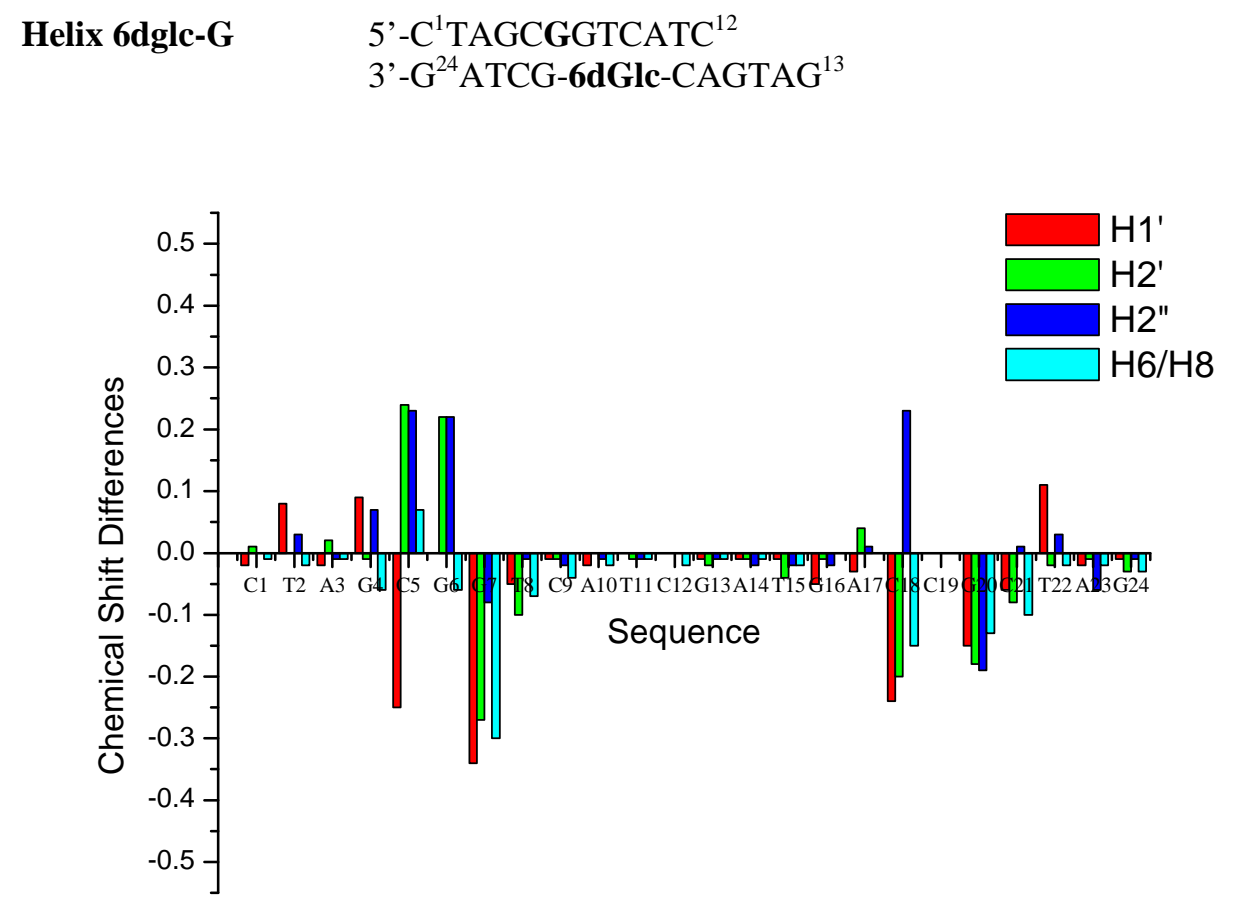

Helix 6dglc-T $\quad 5^{\prime}-G^{1}$ ATGACTGCTAG ${ }^{12}$

$3^{\prime}-C^{24}$ TACTG-6dglc-CGATC ${ }^{13}$

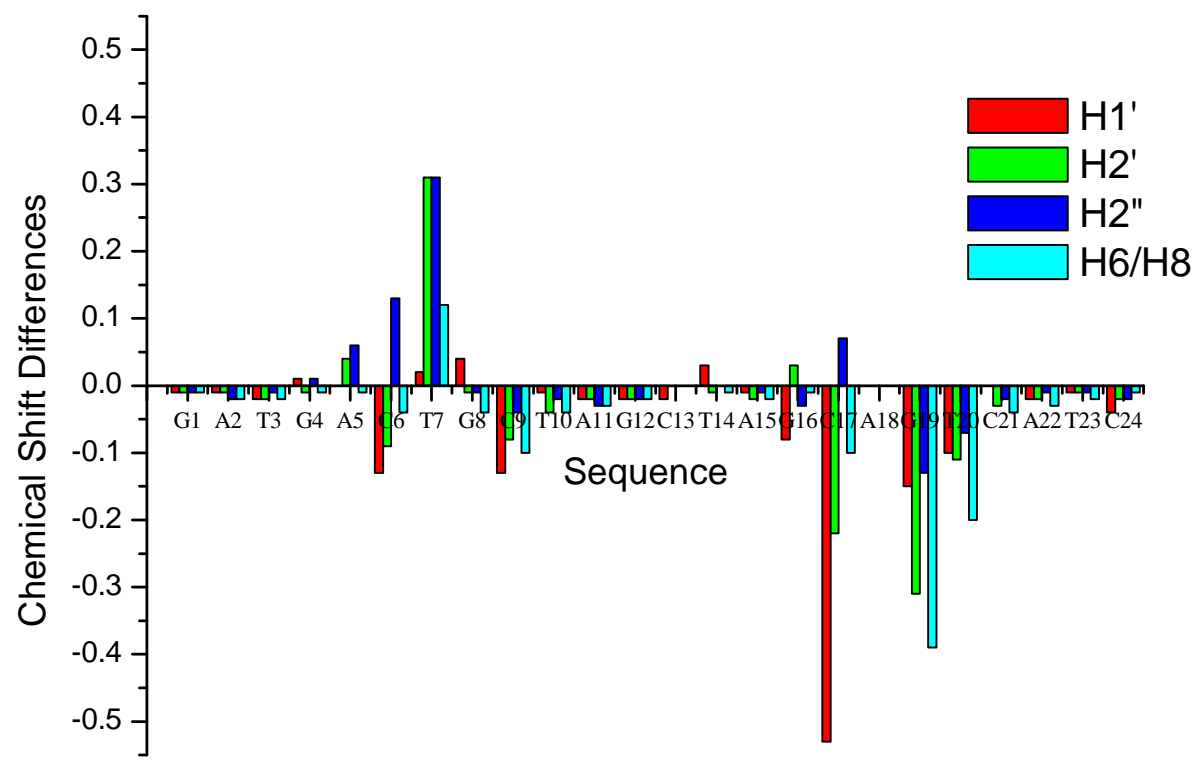


Figure S6.- Several regions of NOESY spectra of helix 6dglc-T in $\mathrm{H}_{2} \mathrm{O} / \mathrm{D}_{2} \mathrm{O}$ 9:1 (left panels) and $\mathrm{D}_{2} \mathrm{O}$ (right panels) (mixing time 100 and $250 \mathrm{~ms}$, respectively, $\mathrm{T}={ }^{\circ} 5 \mathrm{C}$ ). DNA carbohydrate contacts are labeled in red.
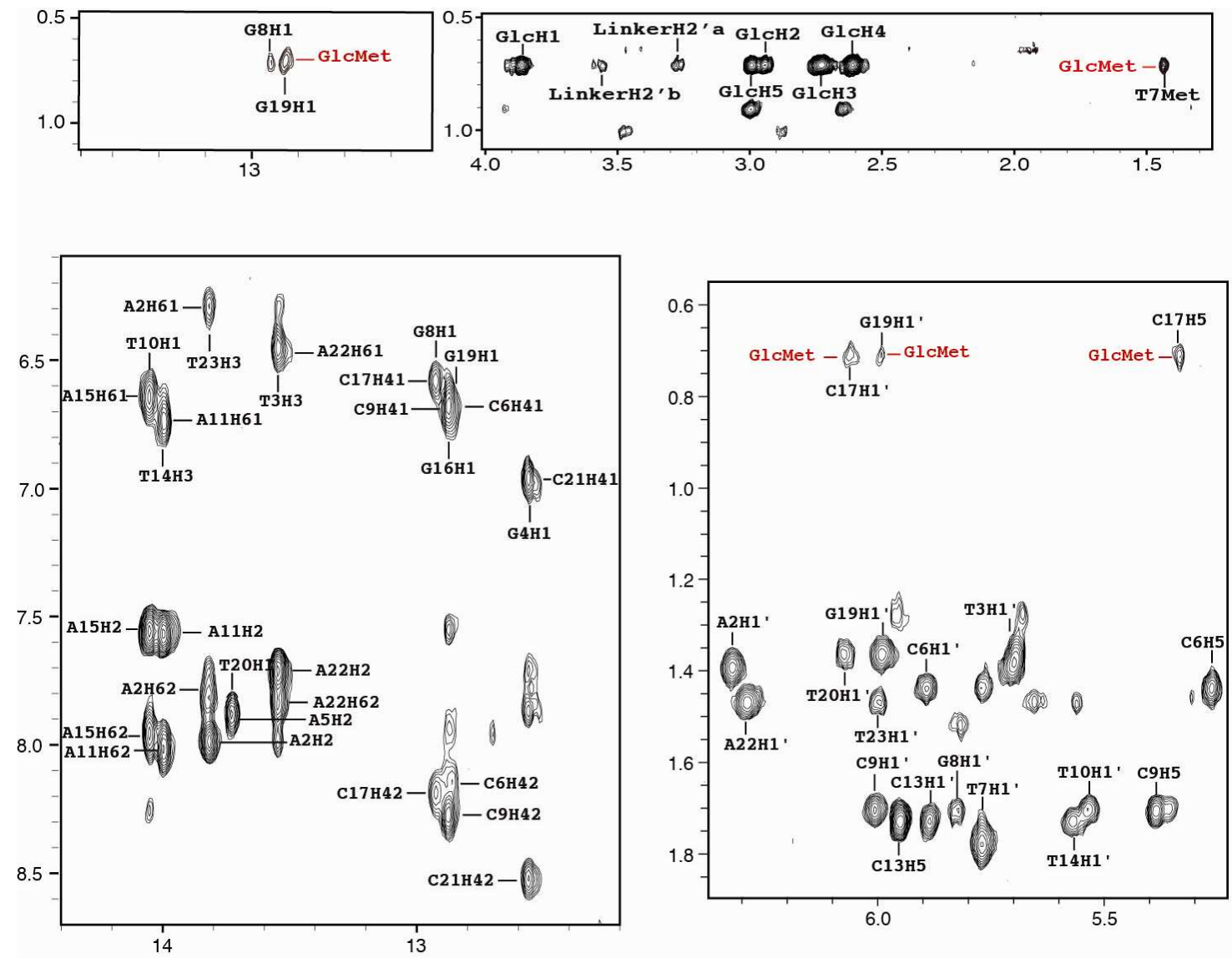
Figure S7. Solution structure of helix 6dglc-T. a) Stereoscopic view of the ensemble of the 10 refined structures, b) Stereoscopic view of a representative structure. Color code: modified strand in green; complementary strand in blue; carbohydrate and linker in magenta; hydrogen atoms are shown in grey. c) Two views showing details of the carbohydrate moiety and the surrounding base-pairs. d) Detail of the interaction between carbohydrate and the opposite thymine. Hydrogen bonds are shown in yellow. 

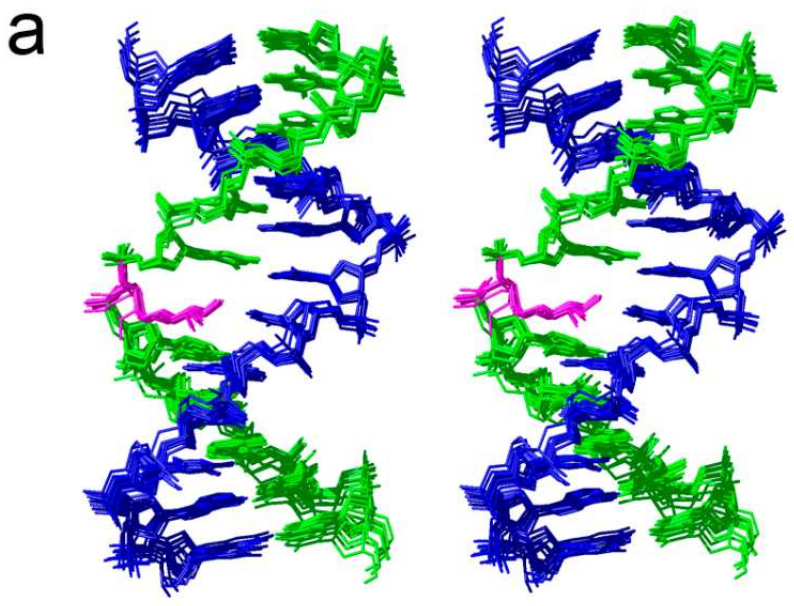

b
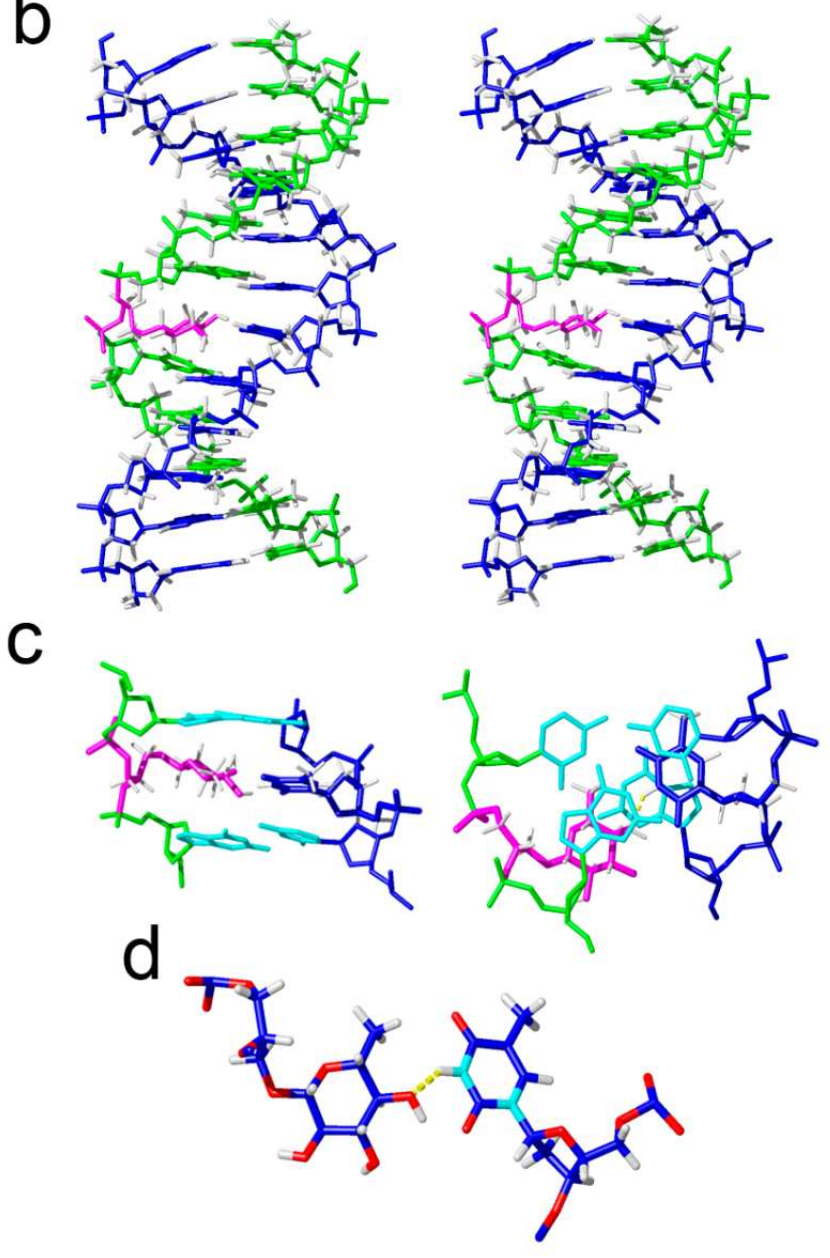

Figure S8. Solution structure of helix 6dglc-G. a) Stereoscopic view of the ensemble of the 10 refined structures, b) Stereoscopic view of a representative structure. Color code: modified strand in green; complementary strand in blue; carbohydrate and linker in magenta; hydrogen atoms are shown in grey. c) Two views showing details of the carbohydrate moiety and the 
surrounding base-pairs. d) Detail of the interaction between carbohydrate and the opposite guanine. Hydrogen bonds are shown in yellow.
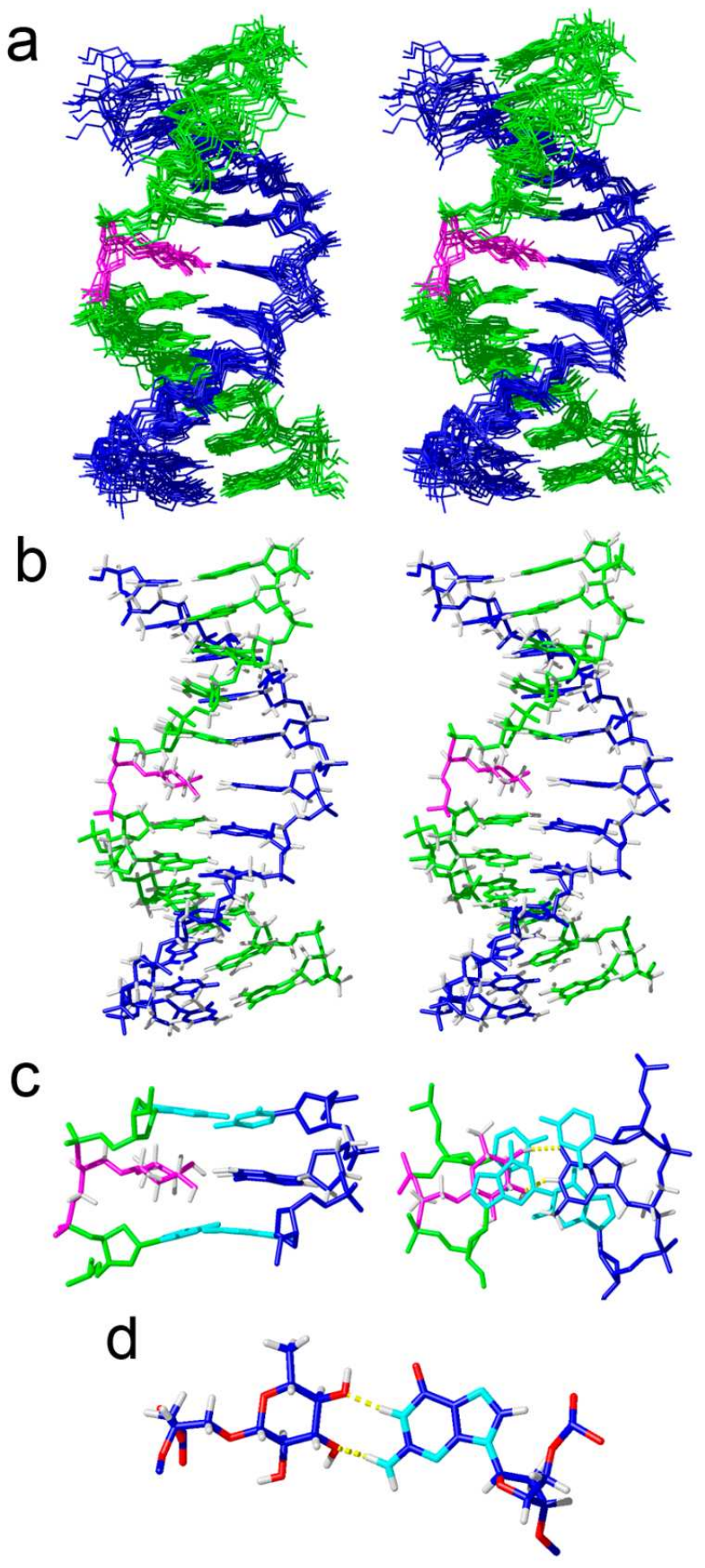

Figure S9. Structures for pairs containing glc and 6dglc in vacuo and in water, computed at BLYP-D3(BJ)/TZ2P using COSMO to simulate aqueous solution. 


6dglc-C


Table S1. ${ }^{1} \mathrm{H}-\mathrm{NMR}$ assignments of helix 6dglc-T $(10 \mathrm{mM}$ sodium phosphate, $150 \mathrm{mM} \mathrm{NaCl}$, $\left.\mathrm{pH} 7, \mathrm{~T}=5^{\circ} \mathrm{C}\right){ }^{+}$

\section{Oligonucleotide strands}

\begin{tabular}{|c|c|c|c|c|c|c|c|c|}
\hline $\begin{array}{c}\text { helix } \\
\text { 6dglc-T }\end{array}$ & H1' & H2'/H2' & H3' & H4' & H5'/H5’ & H5/Met/H2 & H6/H8 & Imino/amino \\
\hline G1 & 5.70 & $2.66 / 2.83$ & 4.88 & 4.23 & 3.72 & --- & 7.96 & n.o. \\
\hline A2 & 6.32 & $2.76 / 2.99$ & 5.08 & 4.49 & $4.23 / 4.16$ & 7.97 & 8.37 & $6.30 / 7.81$ \\
\hline T3 & 5.71 & $1.98 / 2.37$ & 4.88 & 4.17 & 4.30 & 1.40 & 7.15 & 13.55 \\
\hline G4 & 5.54 & $2.69 / 2.75$ & 4.93 & 4.35 & n.a. & n.a. & 7.89 & 12.56 \\
\hline A5 & 6.21 & $2.66 / 2.84$ & 4.77 & 4.47 & $4.24 / 4.15$ & 7.87 & 8.19 & n.o. \\
\hline C6 & 5.90 & $2.01 / 2.34$ & 4.77 & 4.14 & n.a. & 5.27 & 7.32 & n.o. \\
\hline T7 & 5.77 & $1.78 / 2.17$ & 4.76 & 4.11 & 4.02 & 1.44 & 7.16 & 10.48 \\
\hline G8 & 5.83 & 2.68 & 4.93 & 4.34 & $4.05 / 3.96$ & --- & 7.94 & 12.92 \\
\hline C9 & 6.01 & $2.12 / 2.49$ & 4.77 & 4.24 & $4.15 / 4.08$ & 5.39 & 7.53 & $6.68 / 8.28$ \\
\hline T10 & 5.54 & $2.14 / 2.39$ & 4.87 & 4.24 & $4.15 / 4.07$ & 1.71 & 7.49 & 14.04 \\
\hline A11 & 6.11 & $2.93 / 2.76$ & 5.09 & 4.45 & $4.15 / 4.05$ & 7.55 & 8.27 & $6.74 / 8.02$ \\
\hline G12 & 6.03 & $2.47 / 2.27$ & 4.66 & 4.21 & $4.30 / 4.13$ & --- & 7.77 & n.o. \\
\hline C13 & 5.89 & $2.19 / 2.56$ & 4.69 & 4.11 & 3.81 & 5.96 & 7.89 & $7.22 / 7.99$ \\
\hline T14 & 5.57 & $2.26 / 2.53$ & 4.90 & 4.18 & 4.04 & 1.73 & 7.62 & 14.00 \\
\hline A15 & 6.11 & $2.80 / 2.96$ & 5.10 & 4.45 & $4.19 / 4.06$ & 7.55 & 8.27 & $6.65 / 7.56$ \\
\hline G16 & 5.77 & $2.47 / 2.65$ & 4.97 & 4.38 & 4.21 & --- & 7.70 & 12.87 \\
\hline C17 & 6.06 & $2.15 / 2.28$ & 4.81 & n.a. & n.a. & 5.33 & 7.38 & $6.58 / 8.17$ \\
\hline 6dglc18 & --- & --- & --- & --- & --- & --- & --- & --- \\
\hline G19 & 6.00 & $2.76 / 2.84$ & 4.92 & 4.33 & 4.02 & --- & 7.98 & n.o. \\
\hline T20 & 6.08 & $2.19 / 2.54$ & 4.89 & 4.25 & $4.19 / 4.11$ & 1.36 & 7.39 & 13.73 \\
\hline C21 & 5.57 & $2.20 / 2.48$ & 4.88 & n.a. & n.a. & 5.70 & 7.61 & $6.96 / 8.52$ \\
\hline A22 & 6.29 & $2.73 / 2.97$ & 4.87 & 4.46 & 4.16 & 7.70 & 8.40 & $6.46 / 7.85$ \\
\hline T23 & 6.00 & $2.03 / 2.47$ & 4.86 & 4.16 & n.a. & 1.47 & 7.25 & 13.82 \\
\hline C24 & 6.26 & 2.26 & 4.59 & 4.00 & n.a. & 5.65 & 7.58 & n.o. \\
\hline & & & & & & & & \\
\hline
\end{tabular}

6dglc 18

\begin{tabular}{|c|c|c|c|c|c|}
\hline H1 & H2 & H3 & H4 & H5 & Met \\
\hline 3.86 & 2.95 & 2.74 & 2.62 & 2.99 & 0.71 \\
\hline
\end{tabular}

\section{Linker}

\begin{tabular}{|c|c|c|}
\hline H2'/H2' & H5'/H5' & H3' \\
\hline $3.28 / 3.56$ & 3.92 & 4.31 \\
\hline
\end{tabular}

${ }^{+}$n.a. Not assigned. n.o. Not observed. No guanine amino protons could be identified. 
Table S2. ${ }^{1} \mathrm{H}-\mathrm{NMR}$ assignments of helix 6dglc-G (10 mM sodium phosphate, $150 \mathrm{mM} \mathrm{NaCl}$, $\left.\mathrm{pH} 7, \mathrm{~T}=5^{\circ} \mathrm{C}\right)^{+}$

\section{Oligonucleotide strands}

\begin{tabular}{|c|c|c|c|c|c|c|c|c|}
\hline $\begin{array}{c}\text { helix } \\
\text { 6dglc-G }\end{array}$ & H1' & H2'/H2' & H3' & H4' & H5'/H5' & H5/Met/H2 & H6/H8 & Imino/amino \\
\hline C1 & 5.89 & $2.17 / 2.55$ & 4.69 & 4.11 & 3.80 & 5.96 & 7.88 & $7.23 / 8.01$ \\
\hline T2 & 5.50 & $2.26 / 2.48$ & n.a. & 4.16 & 4.01 & 1.74 & 7.61 & 13.82 \\
\hline A3 & 6.10 & $2.91 / 2.78$ & n.a. & 4.43 & $4.14 / 4.07$ & 7.48 & 8.26 & $6.67 / 7.95$ \\
\hline G4 & 5.59 & 2.52 & n.a. & 4.35 & n.a. & --- & 7.77 & 12.75 \\
\hline C5 & 5.92 & $1.58 / 2.09$ & 4.74 & 4.09 & n.a. & 5.44 & 7.12 & $6.47 / 8.13$ \\
\hline G6 & n.a. & 2.48 & n.a. & n.a. & n.a. & --- & 7.89 & 10.32 \\
\hline G7 & 6.00 & $2.77 / 2.82$ & n.a. & 4.40 & n.a. & --- & 7.93 & 12.70 \\
\hline T8 & 6.06 & $2.19 / 2.52$ & n.a. & 4.23 & 4.15 & 1.34 & 7.32 & n.o. \\
\hline C9 & 5.56 & $2.18 / 2.47$ & 4.86 & 4.17 & 4.08 & 5.69 & 7.60 & $6.92 / 8.49$ \\
\hline A10 & 6.28 & $2.71 / 2.95$ & n.a. & 4.45 & 4.15 & 7.66 & 8.38 & $6.44 / 7.82$ \\
\hline T11 & 5.99 & $2.02 / 2.47$ & 4.84 & 4.16 & n.a. & 1.46 & 7.24 & n.o. \\
\hline C12 & 6.25 & 2.25 & 4.58 & 4.00 & 4.20 & 5.69 & 7.58 & n.o. \\
\hline G13 & 5.70 & $2.65 / 2.82$ & 4.88 & 4.23 & 3.71 & --- & 7.95 & 12.68 \\
\hline A14 & 6.31 & $2.75 / 2.98$ & n.a. & 4.48 & $4.23 / 4.15$ & 7.99 & 8.36 & $6.27 / 7.78$ \\
\hline T15 & 5.71 & $1.98 / 2.37$ & 4.88 & 4.17 & n.a. & 1.37 & 7.13 & 13.51 \\
\hline G16 & 5.57 & $2.68 / 2.76$ & n.a. & n.a. & n.a. & --- & 7.87 & 12.51 \\
\hline A17 & 6.24 & $2.62 / 2.89$ & n.a. & n.a. & n.a. & 7.86 & 8.17 & n.o. \\
\hline C18 & 6.00 & 2.14 & 4.84 & n.a. & n.a. & 5.30 & 7.35 & $6.59 / 8.04$ \\
\hline 6dglc19 & --- & --- & --- & --- & --- & --- & --- & --- \\
\hline G20 & 6.08 & $2.84 / 2.92$ & 5.07 & 4.22 & $3.89 / 3.83$ & --- & 8.03 & 13.02 \\
\hline C21 & 5.94 & $2.07 / 2.43$ & 4.80 & 4.18 & n.a. & 5.44 & 7.49 & $6.81 / 8.38$ \\
\hline T22 & 5.42 & $2.12 / 2.33$ & 4.84 & 4.11 & 4.02 & 1.71 & 7.44 & 13.84 \\
\hline A23 & 6.10 & $2.91 / 2.78$ & 5.13 & 4.43 & $4.14 / 4.02$ & n.a. & 8.26 & $6.78 / 8.02$ \\
\hline G24 & 6.02 & $2.26 / 2.48$ & 4.65 & 4.19 & 4.11 & --- & 7.77 & n.o. \\
\hline
\end{tabular}

6dglc 19

\begin{tabular}{|c|c|c|c|c|c|}
\hline H1 & H2 & H3 & H4 & H5 & Met \\
\hline 3.89 & 2.92 & 3.21 & 2.75 & 3.00 & 0.72 \\
\hline
\end{tabular}

Linker

\begin{tabular}{|c|c|c|}
\hline H2'/H2' & H5'/H5' & H3' \\
\hline 3.27 & 3.84 & 4.23 \\
\hline
\end{tabular}


${ }^{+}$n.a. Not assigned. n.o. Not observed. No guanine amino protons could be identified. 
Table S3.Structurally relevant carbohydrate-DNA NOE contacts for helix 6dglc-G and helix 6dglc-T.

\begin{tabular}{|c|c|c|}
\hline $\begin{array}{c}\text { Carbohydrate- } \\
\text { DNA NOEs }\end{array}$ & helix 6dglc-G & helix 6dglc-T \\
\hline DNA-Glc & $\begin{array}{l}\text { G20H1-Met: w } \\
\text { C18H6-Met: w } \\
\text { C18H5-Met: w } \\
\text { C18H1'-Met: w } \\
\text { C18Q2'-Met: w } \\
\text { C5H41-Met: w } \\
\text { C5H42-Met: w }\end{array}$ & $\begin{array}{l}\text { G19H1'-Met: w } \\
\text { G19H8-Met: vw } \\
\text { G19H3-Met: w } \\
\text { G8H3-Met: w } \\
\text { G8H8-Met: vw } \\
\text { C17H6-Met: vw } \\
\text { C17H5-Met: w } \\
\text { C17H1'-Met: w } \\
\text { C17H1'-H2: vw } \\
\text { T7Met-Met: m } \\
\text { T7H6-Met: vw }\end{array}$ \\
\hline DNA-Linker & $\begin{array}{l}\text { G20H8-LinkH2': } \\
\text { vw } \\
\text { C18H1'-LinkH5': } \\
\text { vw }\end{array}$ & $\begin{array}{l}\text { G19H8-LinkH3': w } \\
\text { G19H8-LinkH5': w } \\
\text { G19H8-LinkH2': } \\
\text { vw } \\
\text { G19H8-LinkH2”: } \\
\text { vw } \\
\text { C17H1'-LinkH5': } \\
\text { w } \\
\text { C17H6-LinkH5': } \\
\text { vw }\end{array}$ \\
\hline Linker-Glc & $\begin{array}{l}\text { LinkH5'-Met: vw } \\
\text { LinkH3'-Met: w } \\
\text { LinkH2'-Met: w }\end{array}$ & $\begin{array}{l}\text { LinkH3'-Met: vw } \\
\text { LinkH2'-Met: vw } \\
\text { LinkH2'-Met: vw } \\
\text { LinkH2'-H5: w } \\
\text { LinkH2'-H5: w } \\
\text { LinkH2'-H3: vw } \\
\text { LinkH2'-H3: vw } \\
\text { LinkH2'-H1: w } \\
\text { LinkH2'-H1: w } \\
\text { LinkH3'-H5: vw }\end{array}$ \\
\hline Linker-Linker & $\begin{array}{l}\text { LinkH2' - LinkH5': } \\
\text { w } \\
\text { LinkH2' - LinkH3': } \\
\text { m }\end{array}$ & $\begin{array}{l}\text { LinkH2'- LinkH5': } \\
\text { w } \\
\text { LinkH2"- LinkH5': } \\
\text { w } \\
\text { LinkH2'- LinkH3': } \\
\text { w } \\
\text { LinkH2"'- LinkH3': } \\
\text { w }\end{array}$ \\
\hline
\end{tabular}


Table S4. NMR structural constraints and calculation statistics.

\begin{tabular}{ccc}
\hline $\begin{array}{c}\text { Experimental distance } \\
\text { constraints }\end{array}$ & helix 6dglc-G & helix 6dglc-T \\
\hline Total number & 265 & 409 \\
Intra-residue & 131 & 197 \\
Sequential & 104 & 157 \\
Inter-strand & 16 & 25 \\
\hline r.m.s.d. & & \\
\hline Backbone heavy atoms & $1.5 \pm 0.7 \AA$ & $0.8 \pm 0.3 \AA$ \\
Base heavy atoms & $1.1 \pm 0.4 \AA$ & $0.7 \pm 0.3 \AA$ \\
All heavy atoms & $1.3 \pm 0.6 \AA$ & $0.6 \pm 0.2 \AA$ \\
\hline Residual violations & & \\
\hline Sum of violations $(\AA)$ & 10.1 & $24.9 \AA$ \\
Average \& range & $(10.7-9.5)$ & $(25.5-24.3)$ \\
Maximum violation $(\AA)$ & 0.32 & 0.58 \\
Average NOE energy & 39.3 & 125.1 \\
(kcal/mol) & & $122.1-126.3$ \\
Range of NOE energies & $37.2-42.0$ & \\
(kcal/mol) & &
\end{tabular}

\begin{tabular}{ccc}
\hline Number of NOEs & helix 6dglc-G & helix 6dglc-T \\
\hline Glc-DNA & 7 & 12 \\
Linker-DNA & 2 & 6 \\
Glc-Linker & 3 & 8 \\
Linker-Linker & 2 & 4 \\
\hline
\end{tabular}

Table S5. Cartesian coordinates (in $\AA$ ) and ADF total energies (in $\mathrm{kcal} / \mathrm{mol}$ ) of all stationary points in this study, computed at BLYP-D3(BJ)/TZ2P using COSMO to simulate aqueous solution.

\section{6dglc-G}

6dglc-G, bond energy: $-5781.60 \mathrm{kcal} / \mathrm{mol}$

$\begin{array}{lrrr}1 . \mathrm{C} & 1.553373 & -0.693963 & 2.001937 \\ 2 . \mathrm{H} & 0.961107 & 0.225173 & 2.035264 \\ 3 . \mathrm{H} & 0.945801 & -1.517504 & 2.397427 \\ 4 . \mathrm{H} & 2.436595 & -0.566815 & 2.636743 \\ 5 . \mathrm{O} & 0.093093 & 0.112348 & -0.489178\end{array}$




$\begin{array}{rrrr}6 . \mathrm{H} & -0.891799 & -0.024390 & -0.495512 \\ 7 . \mathrm{O} & 0.107444 & -1.886991 & -2.656886 \\ 8 . \mathrm{H} & -0.284002 & -2.738252 & -2.380522 \\ 9 . \mathrm{O} & 2.702894 & -3.059352 & -3.032877 \\ 10 . \mathrm{H} & 3.349215 & -3.785294 & -2.942433 \\ 11 . \mathrm{O} & 2.748176 & -2.219078 & 0.580544 \\ 12 . \mathrm{O} & -2.622667 & 0.027482 & -0.694938 \\ 13 . \mathrm{C} & -2.419648 & -0.086916 & -4.282840 \\ 14 . \mathrm{N} & -1.469612 & -0.553041 & -5.137389 \\ 15 . \mathrm{H} & -0.754864 & -1.179362 & -4.782242 \\ 16 . \mathrm{H} & -1.733238 & -0.657916 & -6.109583 \\ 17 . \mathrm{N} & -3.517771 & 0.504447 & -4.750371 \\ 18 . \mathrm{C} & -4.337165 & 0.946206 & -3.766511 \\ 19 . \mathrm{N} & -5.537934 & 1.598134 & -3.920432 \\ 20 . \mathrm{H} & 5.948947 & -2.688677 & -0.553804 \\ 21 . \mathrm{H} & 5.282471 & -3.149560 & 1.047390 \\ 22 . \mathrm{N} & -5.229415 & 1.426918 & -1.696879 \\ 23 . \mathrm{O} & 4.095123 & -3.678410 & -0.611368 \\ 24 . \mathrm{H} & -6.972916 & 2.371402 & -2.509941 \\ 25 . \mathrm{C} & 3.309859 & -2.520158 & -0.711637 \\ 26 . \mathrm{H} & 3.918683 & -1.663135 & -1.051643 \\ 27 . \mathrm{C} & 2.179445 & -2.806762 & -1.712865 \\ 28 . \mathrm{H} & 1.626282 & -3.686218 & -1.344807 \\ 29 . \mathrm{C} & 1.239245 & -1.605395 & -1.786552 \\ 30 . \mathrm{H} & 1.757877 & -0.768303 & -2.267395 \\ 31 . \mathrm{N} & -2.153929 & -0.227379 & -2.938269 \\ 32 . \mathrm{H} & -1.295044 & -0.738505 & -2.677526 \\ 33 . \mathrm{C} & -6.031180 & 1.862398 & -2.654878 \\ 34 . \mathrm{C} & 5.395971 & -3.456015 & 0.002259 \\ 35 . \mathrm{C} & -4.155367 & 0.845972 & -2.374476 \\ 36 . \mathrm{C} & -2.976348 & 0.214059 & -1.890369 \\ 37 . \mathrm{C} & 0.768500 & -1.150217 & -0.388661 \\ 38 . \mathrm{H} & 0.094775 & -1.914990 & 0.026216 \\ 39 . \mathrm{C} & 1.971619 & -0.986985 & 0.568012 \\ 40 . \mathrm{H} & 2.605919 & -0.170957 & 0.184788 \\ 41 . \mathrm{H} & -5.980025 & 1.839386 & -4.800553 \\ 42 . \mathrm{H} & 5.925402 & -4.408450 & -0.051234\end{array}$

\section{glc-G}

glc-G, bond energy: $-5925.82 \mathrm{kcal} / \mathrm{mol}$
1. C
$1.531473-0.875436$
2.106333 


$\begin{array}{rrrr}2 . \mathrm{O} & 0.875554 & 0.399589 & 2.300914 \\ 3 . \mathrm{H} & 0.873622 & -1.701502 & 2.416134 \\ 4 . \mathrm{H} & 2.423918 & -0.882688 & 2.738885 \\ 5 . \mathrm{O} & -0.014257 & -0.032391 & -0.290804 \\ 6 . \mathrm{H} & -0.984922 & -0.209814 & -0.456170 \\ 7 . \mathrm{O} & 0.105474 & -1.895593 & -2.609949 \\ 8 . \mathrm{H} & -0.263837 & -2.764397 & -2.358178 \\ 9 . \mathrm{O} & 2.757094 & -2.902871 & -3.051671 \\ 10 . \mathrm{H} & 3.409316 & -3.627713 & -3.013911 \\ 11 . \mathrm{O} & 2.773746 & -2.267786 & 0.606967 \\ 12 . \mathrm{O} & -2.656155 & -0.148616 & -0.762705 \\ 13 . \mathrm{C} & -2.367371 & -0.038184 & -4.342920 \\ 14 . \mathrm{N} & -1.401836 & -0.459170 & -5.203648 \\ 15 . \mathrm{H} & -0.705060 & -1.119744 & -4.877553 \\ 16 . \mathrm{H} & -1.644544 & -0.498224 & -6.186015 \\ 17 . \mathrm{N} & -3.450926 & 0.588308 & -4.798245 \\ 18 . \mathrm{C} & -4.290143 & 0.976358 & -3.808844 \\ 19 . \mathrm{N} & -5.483197 & 1.644131 & -3.950400 \\ 20 . \mathrm{H} & 5.985581 & -2.552510 & -0.526645 \\ 21 . \mathrm{H} & 5.324146 & -3.133061 & 1.037905 \\ 22 . \mathrm{N} & -5.228584 & 1.335777 & -1.734707 \\ 23 . \mathrm{O} & 4.172581 & -3.608469 & -0.661240 \\ 24 . \mathrm{H} & -6.945857 & 2.341983 & -2.529324 \\ 25 . \mathrm{C} & 3.346374 & -2.479523 & -0.704453 \\ 26 . \mathrm{H} & 3.919773 & -1.581020 & -0.993089 \\ 27 . \mathrm{C} & 2.225544 & -2.755656 & -1.720537 \\ 28 . \mathrm{H} & 1.716106 & -3.680165 & -1.405132 \\ 29 . \mathrm{C} & 1.223041 & -1.599933 & -1.728481 \\ 30 . \mathrm{H} & 1.693621 & -0.710929 & -2.162935 \\ 31 . \mathrm{N} & -2.133472 & -0.260209 & -3.004002 \\ 32 . \mathrm{H} & -1.285035 & -0.792125 & -2.747397 \\ 33 . \mathrm{C} & -6.004382 & 1.835075 & -2.682780 \\ 34 . \mathrm{C} & 5.459553 & -3.372412 & -0.021919 \\ 35 . \mathrm{C} & -4.141965 & 0.789869 & -2.421330 \\ 36 . \mathrm{C} & -2.979525 & 0.119086 & -1.952732 \\ 37 . \mathrm{C} & 0.742782 & -1.260580 & -0.303456 \\ 38 . \mathrm{H} & 0.125097 & -2.089914 & 0.072341 \\ 39 . \mathrm{C} & 1.949969 & -1.079740 & 0.644486 \\ 40 . \mathrm{H} & 2.535293 & -0.206967 & 0.311950 \\ 41 . \mathrm{H} & -5.902679 & 1.940839 & -4.824450 \\ \text { 43.H } & 6.025515 & -4.299210 & -0.126008 \\ \mathrm{C}-\mathrm{T} & 0.285233 & 0.508883 & 1.520163\end{array}$

\section{6dgle-T}


6dglc-T, bond energy: $-5472.38 \mathrm{kcal} / \mathrm{mol}$

\begin{tabular}{|c|c|c|c|}
\hline 1. $\mathrm{H}$ & 3.193367 & 1.449423 & -0.360756 \\
\hline 2. C & 6.309905 & 0.268397 & -0.467993 \\
\hline 3. C & 3.566909 & -0.560656 & -0.380522 \\
\hline $4 . \mathrm{H}$ & -4.286484 & 0.878373 & -1.798914 \\
\hline $5 . \mathrm{H}$ & 4.391489 & -2.427264 & -0.413511 \\
\hline $6 . \mathrm{N}$ & 4.622933 & -1.439199 & -0.416949 \\
\hline 7. C & 7.746799 & 0.716355 & -0.519789 \\
\hline $8 . N$ & 3.945002 & 0.764093 & -0.385093 \\
\hline $9 . \mathrm{H}$ & 8.419853 & -0.145817 & -0.537032 \\
\hline $10 . \mathrm{H}$ & 7.993623 & 1.337345 & 0.349912 \\
\hline 11. C & 5.941553 & -1.041224 & -0.461942 \\
\hline $12 . \mathrm{H}$ & 6.661026 & -1.851104 & -0.491523 \\
\hline 13. C & -3.025128 & 5.600927 & -0.626714 \\
\hline $14 . \mathrm{H}$ & -3.651591 & 5.451549 & 0.262129 \\
\hline $15 . \mathrm{H}$ & -3.488563 & 6.334421 & -1.288474 \\
\hline 16.0 & -2.936694 & 4.364418 & -1.389220 \\
\hline 17. C & -2.440101 & 3.283256 & -0.649792 \\
\hline $18 . \mathrm{H}$ & -2.896563 & 3.253228 & 0.355515 \\
\hline 19. C & -2.721069 & 1.982684 & -1.406881 \\
\hline $20 . \mathrm{H}$ & -2.261722 & 2.068771 & -2.404685 \\
\hline $21 . \mathrm{C}$ & -2.093250 & 0.796070 & -0.656566 \\
\hline 22.0 & -1.008473 & 3.440470 & -0.513114 \\
\hline $23 . \mathrm{H}$ & -2.601777 & 0.681573 & 0.309136 \\
\hline $24 . \mathrm{C}$ & -0.598108 & 1.023472 & -0.396067 \\
\hline $25 . \mathrm{H}$ & -0.059682 & 1.035139 & -1.356971 \\
\hline $26 . \mathrm{C}$ & -0.398944 & 2.396205 & 0.296615 \\
\hline $27 . \mathrm{H}$ & -0.903774 & 2.360200 & 1.275493 \\
\hline $28 . \mathrm{C}$ & 1.067210 & 2.762466 & 0.481918 \\
\hline 29. H & 1.559890 & 2.014671 & 1.111918 \\
\hline $30 . \mathrm{H}$ & 1.162605 & 3.736440 & 0.972087 \\
\hline $31 . \mathrm{C}$ & 5.257648 & 1.274163 & -0.425213 \\
\hline 32.0 & 2.379991 & -0.937047 & -0.348943 \\
\hline 33.0 & 5.432650 & 2.505135 & -0.423007 \\
\hline 34.0 & -4.148544 & 1.807679 & -1.530649 \\
\hline $35 . \mathrm{H}$ & 1.567777 & 2.815650 & -0.494401 \\
\hline 36.0 & -0.107500 & -0.042941 & 0.434751 \\
\hline $37 . \mathrm{H}$ & 0.809691 & -0.271380 & 0.149699 \\
\hline 38.0 & -2.370576 & -0.436760 & -1.362635 \\
\hline $39 . \mathrm{H}$ & -1.869808 & -0.424791 & -2.20123 \\
\hline $40 . \mathrm{H}$ & -2.029580 & 5.944811 & -0.326444 \\
\hline $41 . \mathrm{H}$ & 7.933948 & 1.324859 & -1.41312 \\
\hline
\end{tabular}




\section{glc-T}

glc-T, bond energy: $-5617.61 \mathrm{kcal} / \mathrm{mol}$

\begin{tabular}{|c|c|c|c|}
\hline 1. $\mathrm{H}$ & 3.417264 & 1.705725 & 0.063497 \\
\hline 2. $\mathrm{C}$ & 6.518743 & 0.432748 & -0.121675 \\
\hline 3. C & 3.825846 & -0.026863 & -0.964578 \\
\hline $4 . \mathrm{H}$ & -4.371765 & 0.516456 & -0.877370 \\
\hline $5 . \mathrm{H}$ & 4.648887 & -1.667552 & -1.862628 \\
\hline $6 . N$ & 4.871691 & -0.856467 & -1.294749 \\
\hline 7. C & 7.930033 & 0.692800 & 0.334072 \\
\hline $8 . \mathrm{N}$ & 4.179012 & 1.053708 & -0.193295 \\
\hline 9. H & 8.606297 & -0.084531 & -0.033620 \\
\hline 10. H & 7.987155 & 0.718176 & 1.429539 \\
\hline 11. C & 6.170285 & -0.634224 & -0.889800 \\
\hline $12 . \mathrm{H}$ & 6.887207 & -1.373845 & -1.226662 \\
\hline 13. C & -3.474356 & 5.462786 & -0.495675 \\
\hline $14 . \mathrm{H}$ & -3.758409 & 5.361599 & 0.559573 \\
\hline 15. H & -4.217774 & 6.060184 & -1.025704 \\
\hline 16.0 & -3.469633 & 4.156641 & -1.139508 \\
\hline 17. C & -2.631551 & 3.228067 & -0.514234 \\
\hline $18 . \mathrm{H}$ & -2.755294 & 3.262564 & 0.582929 \\
\hline 19. C & -2.931736 & 1.826933 & -1.049109 \\
\hline $20 . \mathrm{H}$ & -2.805123 & 1.850426 & -2.143126 \\
\hline $21 . \mathrm{C}$ & -1.944703 & 0.816348 & -0.440731 \\
\hline 22.0 & -1.259409 & 3.559314 & -0.840195 \\
\hline $23 . \mathrm{H}$ & -2.128216 & 0.752017 & 0.639225 \\
\hline $24 . \mathrm{C}$ & -0.483283 & 1.239406 & -0.660915 \\
\hline $25 . \mathrm{H}$ & -0.255411 & 1.201416 & -1.738038 \\
\hline $26 . \mathrm{C}$ & -0.296222 & 2.701415 & -0.177765 \\
\hline $27 . \mathrm{H}$ & -0.457051 & 2.735364 & 0.913303 \\
\hline $28 . \mathrm{C}$ & 1.075541 & 3.314982 & -0.497235 \\
\hline 29.0 & 2.118210 & 2.889188 & 0.410551 \\
\hline $30 . \mathrm{H}$ & 0.973671 & 4.406660 & -0.469464 \\
\hline 31. C & 5.468564 & 1.364591 & 0.269025 \\
\hline 32.0 & 2.655212 & -0.246239 & -1.343507 \\
\hline 33.0 & 5.642193 & 2.384407 & 0.963318 \\
\hline 34.0 & -4.289930 & 1.475818 & -0.712234 \\
\hline $35 . \mathrm{H}$ & 1.410953 & 3.019151 & -1.495750 \\
\hline 36.0 & 0.361650 & 0.330939 & 0.060122 \\
\hline $37 . \mathrm{H}$ & 1.209391 & 0.213424 & -0.438569 \\
\hline 38.0 & -2.231247 & -0.511737 & -0.938349 \\
\hline $39 . \mathrm{H}$ & -2.006869 & -0.534120 & -1.888735 \\
\hline $40 . \mathrm{H}$ & -2.489815 & 5.936007 & -0.572358 \\
\hline
\end{tabular}



$41 . \mathrm{H}$
1.832554
3.060725
1. 325935
$42 . \mathrm{H}$
8.284046
1.666370
$-0.027402$

6dglc-A

6dglc-A, bond energy: $-5616.87 \mathrm{kcal} / \mathrm{mol}$

$\begin{array}{rrrr}\text { 1.C } & 1.932190 & -1.132459 & 2.376285 \\ 2 . \mathrm{H} & 1.171714 & -0.446386 & 2.763926 \\ 3 . \mathrm{H} & 1.772750 & -2.122491 & 2.821599 \\ 4 . \mathrm{H} & 2.916529 & -0.760216 & 2.679817 \\ 5 . \mathrm{O} & -0.576901 & -0.808133 & 0.692904 \\ 6 . \mathrm{H} & -0.911375 & -1.029367 & 1.579040 \\ 7 . \mathrm{O} & -0.732394 & -2.559613 & -1.607162 \\ 8 . \mathrm{H} & -2.126026 & -1.791863 & -4.536102 \\ 9 . \mathrm{O} & 1.728921 & -2.706194 & -3.072252 \\ 10 . \mathrm{H} & 2.552377 & -3.162137 & -3.330283 \\ 11 . \mathrm{O} & 2.891862 & -2.111584 & 0.401146 \\ 12 . \mathrm{N} & -2.577137 & 0.619622 & -0.878824 \\ 13 . \mathrm{C} & -2.623582 & -0.931705 & -4.095340 \\ 14 . \mathrm{C} & 1.842881 & -1.210651 & 0.858082 \\ 15 . \mathrm{H} & 2.010344 & -0.214206 & 0.417448 \\ 16 . \mathrm{H} & -5.331493 & 1.746853 & -5.650225 \\ 17 . \mathrm{N} & -3.463031 & -0.251908 & -4.889393 \\ 18 . \mathrm{C} & -4.027154 & 0.793577 & -4.242105 \\ 19 . \mathrm{N} & -4.935869 & 1.715529 & -4.717206 \\ 20 . \mathrm{H} & 5.425316 & -1.453334 & -1.781941 \\ 21 . \mathrm{H} & 5.550712 & -2.216736 & -0.163047 \\ 22 . \mathrm{N} & -4.544825 & 2.295430 & -2.574224 \\ 23 . \mathrm{O} & 4.038157 & -2.988904 & -1.410516 \\ 24 . \mathrm{H} & -5.901457 & 3.408388 & -3.806194 \\ 25 . \mathrm{C} & 2.978160 & -2.145272 & -1.038825 \\ 26 . \mathrm{H} & 3.148169 & -1.119906 & -1.413505 \\ 27 . \mathrm{C} & 1.685152 & -2.726371 & -1.628702 \\ 28 . \mathrm{H} & 1.581297 & -3.759845 & -1.264679 \\ 29 . \mathrm{C} & 0.469292 & -1.912236 & -1.175227 \\ 30 . \mathrm{H} & 0.535259 & -0.911987 & -1.633037 \\ 31 . \mathrm{N} & -2.313704 & -0.683746 & -2.804583 \\ 32 . \mathrm{H} & 6.072038 & -3.117528 & -1.619698 \\ 33 . \mathrm{C} & -5.210732 & 2.586681 & -3.684473 \\ 34 . \mathrm{C} & 5.354171 & -2.396397 & -1.225357 \\ 35 . \mathrm{C} & -3.794827 & 1.167611 & -2.907929 \\ 36 . \mathrm{C} & -2.886579 & 0.376346 & -2.165009\end{array}$




$\begin{array}{lrrr}\text { 37.C } & 0.482921 & -1.741081 & 0.353620 \\ 38 . \mathrm{H} & 0.280290 & -2.714878 & 0.819008 \\ 39 . \mathrm{H} & -2.974149 & 1.426873 & -0.416231 \\ 40 . \mathrm{H} & -1.883973 & 0.056283 & -0.381002 \\ 41 . \mathrm{H} & -1.323139 & -1.861717 & -2.031931\end{array}$

glc-A

glc-A, bond energy: $-5760.59 \mathrm{kcal} / \mathrm{mol}$

$\begin{array}{rrrr}\text { 1.C } & 2.029877 & -0.824807 & 2.307484 \\ 2 . \mathrm{O} & 1.116610 & 0.150877 & 2.863258 \\ 3 . \mathrm{H} & 1.936468 & -1.787655 & 2.831823 \\ 4 . \mathrm{H} & 3.041971 & -0.439122 & 2.460154 \\ 5 . \mathrm{O} & -0.638745 & -0.961206 & 0.963178 \\ 6 . \mathrm{H} & -1.366984 & -1.570606 & 1.180659 \\ 7 . \mathrm{O} & -0.857025 & -2.792849 & -1.271278 \\ 8 . \mathrm{H} & -2.681132 & -2.424561 & -4.011743 \\ 9 . \mathrm{O} & 1.483873 & -2.902152 & -2.957505 \\ 10 . \mathrm{H} & 2.307238 & -3.330407 & -3.259137 \\ 11 . \mathrm{O} & 2.889651 & -1.845194 & 0.319627 \\ 12 . \mathrm{N} & -2.173408 & 0.735060 & -0.978068 \\ 13 . \mathrm{C} & -2.924328 & -1.398381 & -3.747669 \\ 14 . \mathrm{C} & 1.792688 & -1.035164 & 0.806503 \\ 15 . \mathrm{H} & 1.792465 & -0.055648 & 0.300543 \\ 16 . \mathrm{H} & -5.106955 & 1.481484 & -5.715041 \\ 17 . \mathrm{N} & -3.666886 & -0.712366 & -4.627587 \\ 18 . \mathrm{C} & -3.909658 & 0.548127 & -4.201386 \\ 19 . \mathrm{N} & -4.633245 & 1.545527 & -4.820835 \\ 20 . \mathrm{H} & 5.114580 & -1.150333 & -2.171324 \\ 21 . \mathrm{H} & 5.476017 & -1.713180 & -0.505560 \\ 22 . \mathrm{N} & -3.898626 & 2.438796 & -2.885700 \\ 23 . \mathrm{O} & 3.958514 & -2.777796 & -1.509124 \\ 24 . \mathrm{H} & -5.096361 & 3.569575 & -4.259062 \\ 25 . \mathrm{C} & 2.846036 & -2.021740 & -1.119061 \\ 26 . \mathrm{H} & 2.859485 & -1.026265 & -1.597740 \\ 27 . \mathrm{C} & 1.577143 & -2.792631 & -1.522110 \\ 28 . \mathrm{H} & 1.621983 & -3.791733 & -1.063052 \\ 29 . \mathrm{C} & 0.338505 & -2.049247 & -1.013231 \\ 30 . \mathrm{H} & 0.286314 & -1.081460 & -1.537183 \\ 31 . \mathrm{N} & -2.432732 & -0.974903 & -2.562671 \\ 32 . \mathrm{H} & 5.966439 & -2.706302 & -1.912345 \\ 33 . \mathrm{C} & -4.595637 & 2.648945 & -3.995432\end{array}$




$\begin{array}{lrrr}\text { 34.C } & 5.207373 & -2.028240 & -1.519356 \\ 35 . \mathrm{C} & -3.459700 & 1.119382 & -2.999383 \\ 36 . \mathrm{C} & -2.677623 & 0.301335 & -2.148991 \\ 37 . \mathrm{C} & 0.479786 & -1.775583 & 0.494159 \\ 38 . \mathrm{H} & 0.475623 & -2.729612 & 1.036977 \\ 39 . \mathrm{H} & -2.368032 & 1.680030 & -0.673991 \\ 40 . \mathrm{H} & -1.624744 & 0.128683 & -0.367538 \\ 41 . \mathrm{H} & -1.492110 & -2.155868 & -1.728082 \\ 42 . \mathrm{H} & 0.229215 & -0.080050 & 2.513135\end{array}$

\section{6dglc-C}

6dglc-C, bond energy: $-5215.92 \mathrm{kcal} / \mathrm{mol}$

$\begin{array}{rrrr}\text { 1.H } & 4.220123 & 3.481263 & -0.883081 \\ \text { 2.C } & 6.115123 & 0.799535 & -0.313176 \\ \text { 3.C } & 3.586722 & -0.301652 & -0.738606 \\ 4 . \mathrm{H} & -4.016383 & 0.623446 & -1.688860 \\ \text { 5.H } & 4.576892 & -2.094620 & -0.421461 \\ 6 . \mathrm{N} & 4.712700 & -1.089482 & -0.469970 \\ 7 . \mathrm{O} & -1.914030 & -0.406217 & -1.394477 \\ 8 . \mathrm{N} & 3.737604 & 1.041323 & -0.816650 \\ 9 . \mathrm{H} & -1.458220 & -0.308907 & -2.252756 \\ 10 . \mathrm{H} & -2.416919 & 5.931475 & -0.188635 \\ 11 . \mathrm{C} & 5.947940 & -0.551086 & -0.261312 \\ 12 . \mathrm{H} & 6.750609 & -1.248695 & -0.057915 \\ 13 . \mathrm{C} & -3.373276 & 5.453637 & -0.426256 \\ 14 . \mathrm{H} & -3.900710 & 5.195952 & 0.501239 \\ 15 . \mathrm{H} & -3.986844 & 6.126983 & -1.027164 \\ 16 . \mathrm{O} & -3.168840 & 4.260958 & -1.233398 \\ 17 . \mathrm{C} & -2.472808 & 3.243401 & -0.565097 \\ 18 . \mathrm{H} & -2.856301 & 3.118745 & 0.463439 \\ 19 . \mathrm{C} & -2.609050 & 1.938082 & -1.352084 \\ 20 . \mathrm{H} & -2.223908 & 2.116314 & -2.369029 \\ 21 . \mathrm{C} & -1.776308 & 0.841595 & -0.670949 \\ 22 . \mathrm{O} & -1.075381 & 3.604530 & -0.508931 \\ 23 . \mathrm{H} & -2.208521 & 0.636324 & 0.316551 \\ 24 . \mathrm{C} & -0.311631 & 1.266817 & -0.477453 \\ 25 . \mathrm{H} & 0.173449 & 1.360111 & -1.461652 \\ 26 . \mathrm{C} & -0.260193 & 2.645856 & 0.228413 \\ 27 . \mathrm{H} & -0.682599 & 2.523387 & 1.239372 \\ 28 . \mathrm{C} & 1.145072 & 3.224290 & 0.312428 \\ 29 . \mathrm{H} & 5.924699 & 3.406707 & -0.531164\end{array}$




$\begin{array}{lrrr}30 . \mathrm{H} & 1.136398 & 4.168068 & 0.868237 \\ 31 . \mathrm{C} & 4.950700 & 1.586911 & -0.603816 \\ 32 . \mathrm{O} & 2.476153 & -0.885469 & -0.895269 \\ 33 . \mathrm{N} & 5.044272 & 2.931563 & -0.675786 \\ 34 . \mathrm{O} & -4.002069 & 1.559540 & -1.409218 \\ 35 . \mathrm{H} & 1.539495 & 3.403438 & -0.695115 \\ 36 . \mathrm{O} & 0.365399 & 0.281957 & 0.315929 \\ 37 . \mathrm{H} & 1.154061 & -0.059543 & -0.189667 \\ 38 . \mathrm{H} & 7.084260 & 1.255769 & -0.148762 \\ 39 . \mathrm{H} & 1.812231 & 2.517693 & 0.813370\end{array}$

\section{glc-C}

glc-C, bond energy: $-5358.52 \mathrm{kcal} / \mathrm{mol}$

$\begin{array}{rrrr}\text { 1.H } & 1.117460 & 2.616950 & 2.807804 \\ \text { 2.C } & 5.798065 & 1.142028 & -0.632465 \\ 3 . \mathrm{C} & 3.453603 & -0.371973 & -0.588999 \\ 4 . \mathrm{H} & -3.849285 & 0.947453 & -2.136733 \\ 5 . \mathrm{H} & 4.703593 & -1.848348 & 0.148934 \\ 6 . \mathrm{N} & 4.688190 & -0.888236 & -0.180743 \\ 7 . \mathrm{O} & -1.942304 & -0.354955 & -1.625446 \\ 8 . \mathrm{N} & 3.404551 & 0.901141 & -1.050141 \\ 9 . \mathrm{H} & -1.354114 & -0.262067 & -2.399733 \\ 10 . \mathrm{H} & -1.849685 & 5.926788 & -0.079530 \\ 11 . \mathrm{C} & 5.833183 & -0.148601 & -0.199336 \\ 12 . \mathrm{H} & 6.733101 & -0.643687 & 0.143050 \\ 13 . \mathrm{C} & -2.810618 & 5.593267 & -0.485183 \\ 14 . \mathrm{H} & -3.503409 & 5.369422 & 0.336060 \\ 15 . \mathrm{H} & -3.233225 & 6.367503 & -1.127562 \\ 16 . \mathrm{O} & -2.635894 & 4.418501 & -1.326208 \\ 17 . \mathrm{C} & -2.194751 & 3.288439 & -0.626434 \\ 18 . \mathrm{H} & -2.751824 & 3.171092 & 0.320221 \\ 19 . \mathrm{C} & -2.363596 & 2.049287 & -1.508041 \\ 20 . \mathrm{H} & -1.811893 & 2.225492 & -2.445271 \\ 21 . \mathrm{C} & -1.777943 & 0.820007 & -0.795450 \\ 22 . \mathrm{O} & -0.789609 & 3.459043 & -0.324212 \\ 23 . \mathrm{H} & -2.377803 & 0.611543 & 0.099563 \\ 24 . \mathrm{C} & -0.322147 & 1.056275 & -0.363130 \\ 25 . \mathrm{H} & 0.307200 & 1.180393 & -1.257893 \\ 26 . \mathrm{C} & -0.242392 & 2.365229 & 0.462001 \\ 27 . \mathrm{H} & -0.835496 & 2.243567 & 1.383883\end{array}$




$\begin{array}{rrrr}28 . \mathrm{C} & 1.191329 & 2.781192 & 0.839358 \\ 29 . \mathrm{O} & 1.640317 & 2.211929 & 2.092133 \\ 30 . \mathrm{H} & 1.225271 & 3.877632 & 0.881388 \\ 31 . \mathrm{C} & 4.527675 & 1.646286 & -1.068399 \\ 32 . \mathrm{O} & 2.439851 & -1.121689 & -0.508269 \\ 33 . \mathrm{N} & 4.420528 & 2.914786 & -1.515134 \\ 34 . \mathrm{O} & -3.767268 & 1.856053 & -1.786739 \\ 35 . \mathrm{H} & 1.898108 & 2.423661 & 0.086873 \\ 36.0 & 0.136048 & -0.061827 & 0.405917 \\ 37 . \mathrm{H} & 0.997093 & -0.385356 & 0.019917 \\ 38 . \mathrm{H} & 6.691399 & 1.755096 & -0.655760 \\ 39 . \mathrm{H} & 3.529929 & 3.257819 & -1.852232 \\ 40 . \mathrm{H} & 5.227353 & 3.521293 & -1.571873\end{array}$

\section{6dglc-6dglc}

$\begin{array}{rrrr}6 \mathrm{dglC}-6 \mathrm{dglC}, \text { bond energy: } & -6571.16 \mathrm{kcal} / \mathrm{mol} \\ \text { 1.H } & -1.864818 & -2.231176 & 2.269872 \\ 2 . \mathrm{H} & -0.210374 & 1.820896 & -0.939832 \\ 3 . \mathrm{H} & -6.401935 & 1.544664 & -0.740885 \\ \text { 4.C } & -1.098019 & 1.323729 & -1.343265 \\ 5 . \mathrm{H} & -1.185270 & -0.975844 & 0.232862 \\ 6 . \mathrm{O} & -5.430792 & -0.313663 & -0.578363 \\ 7 . \mathrm{C} & -2.055335 & 0.958975 & -0.216563 \\ 8 . \mathrm{O} & -4.902625 & -1.080105 & 2.106952 \\ 9 . \mathrm{H} & -4.550625 & 1.122602 & 0.624161 \\ 10 . \mathrm{H} & -3.673430 & -1.818968 & 0.578917 \\ 11 . \mathrm{H} & -4.497737 & -1.584943 & 2.838980 \\ 12 . \mathrm{C} & -6.103940 & 0.700215 & -1.375524 \\ 13.0 & -3.247296 & 0.394574 & -0.831731 \\ 14 . \mathrm{C} & -2.556882 & -0.431654 & 1.816331 \\ 15 . \mathrm{H} & -2.335226 & 1.865870 & 0.344118 \\ 16 . \mathrm{H} & 0.457128 & 0.320553 & 0.939474 \\ 17 . \mathrm{H} & -2.768522 & 0.455598 & 2.425914 \\ 18 . \mathrm{C} & -1.475754 & -0.070498 & 0.789861 \\ 19 . \mathrm{C} & -4.314128 & 0.170274 & 0.116501 \\ 20 . \mathrm{H} & -5.454032 & 1.054715 & -2.182418 \\ 21.0 & -2.133975 & -1.436117 & 2.769001 \\ 22 . \mathrm{H} & -6.989573 & 0.219579 & -1.794275 \\ 23 . \mathrm{H} & -1.584081 & 2.013050 & -2.041457 \\ 24 . \mathrm{H} & -0.795973 & 0.424538 & -1.896850 \\ 25 . \mathrm{C} & -3.858468 & -0.882784 & 1.130143\end{array}$




\begin{tabular}{lrrr}
$26 . \mathrm{O}$ & -0.340961 & 0.461117 & 1.498329 \\
$27 . \mathrm{H}$ & 3.672831 & 1.606682 & -1.374778 \\
$28 . \mathrm{C}$ & 3.157992 & -0.285888 & -0.481044 \\
$29 . \mathrm{H}$ & 3.201424 & -1.354614 & -0.741490 \\
$30 . \mathrm{C}$ & 3.933266 & -0.070084 & 0.828613 \\
$31 . \mathrm{H}$ & 3.899090 & 0.999571 & 1.088303 \\
$32 . \mathrm{C}$ & 3.417398 & -0.910858 & 1.987100 \\
$33 . \mathrm{H}$ & 2.379662 & -0.645896 & 2.214875 \\
$34 . \mathrm{H}$ & 3.458258 & -1.977271 & 1.733660 \\
$35 . \mathrm{H}$ & 4.024516 & -0.732262 & 2.880625 \\
$36 . \mathrm{H}$ & 6.764904 & 0.864994 & -2.845962 \\
$37 . \mathrm{C}$ & 8.169992 & 0.377545 & 0.505087 \\
$38 . \mathrm{O}$ & 7.285333 & -0.054605 & -0.567375 \\
$39 . \mathrm{H}$ & 8.151618 & 1.471446 & 0.591440 \\
$40 . \mathrm{H}$ & 7.874403 & -0.077056 & 1.456456 \\
$41 . \mathrm{O}$ & 5.315936 & -0.457112 & 0.581200 \\
$42 . \mathrm{O}$ & 3.008225 & 0.372502 & -2.835703 \\
$43 . \mathrm{C}$ & 3.773194 & 0.542043 & -1.619235 \\
$44 . \mathrm{H}$ & 3.136259 & -0.542895 & -3.152363 \\
$45 . \mathrm{C}$ & 5.957848 & 0.369877 & -0.417092 \\
$46 . \mathrm{H}$ & 9.170760 & 0.042927 & 0.227045 \\
$47 . \mathrm{C}$ & 5.256119 & 0.207608 & -1.779833 \\
$48 . \mathrm{O}$ & 5.820982 & 1.098027 & -2.761464 \\
$49 . \mathrm{H}$ & 5.371961 & -0.840618 & -2.097352 \\
$50 . \mathrm{H}$ & 5.916237 & 1.424371 & -0.090838 \\
$51 . \mathrm{O}$ & 1.776935 & 0.110934 & -0.305424 \\
$52 . \mathrm{H}$ & 1.399736 & 0.177152 & -1.204002 \\
& & & \\
\hline 3 & & &
\end{tabular}

\section{$\underline{\text { 6dglc-glc }}$}

dglc-glc, bond energy: $-6715.32 \mathrm{kcal} / \mathrm{mol}$

$\begin{array}{rrrr}1 . \mathrm{H} & -1.448289 & -2.378187 & 1.870696 \\ 2 . \mathrm{H} & -0.448132 & 2.083572 & -1.128434 \\ 3 . \mathrm{H} & -6.488170 & 1.341717 & -0.275126 \\ 4 . \mathrm{C} & -1.300901 & 1.494341 & -1.481041 \\ 5 . \mathrm{H} & -1.072554 & -0.921194 & -0.107895 \\ 6.0 & -5.429554 & -0.470766 & -0.404066 \\ 7 . \mathrm{C} & -2.110195 & 0.975058 & -0.299616 \\ 8.0 & -4.557514 & -1.432892 & 2.123365 \\ 9 . \mathrm{H} & -4.484683 & 0.908674 & 0.814440 \\ 10 . \mathrm{H} & -3.464132 & -1.959293 & 0.415294 \\ 11 . \mathrm{H} & -4.045317 & -1.960375 & 2.766859\end{array}$




\begin{tabular}{|c|c|c|c|}
\hline 12. C & -6.239616 & 0.571891 & -1.016987 \\
\hline 13.0 & -3.323308 & 0.377261 & -0.833322 \\
\hline 14. C & -2.300253 & -0.603859 & 1.648594 \\
\hline 15. H & -2.384549 & 1.811853 & 0.362519 \\
\hline 16. H & 0.489921 & 0.648779 & 0.491811 \\
\hline $17 . \mathrm{H}$ & -2.497304 & 0.216146 & 2.350266 \\
\hline 18. C & -1.365835 & -0.087233 & 0.549973 \\
\hline 19. C & -4.264321 & 0.015355 & 0.202956 \\
\hline $20 . \mathrm{H}$ & -5.710918 & 1.027976 & -1.860615 \\
\hline 21.0 & -1.711247 & -1.645835 & 2.460689 \\
\hline $22 . \mathrm{H}$ & -7.151255 & 0.084897 & -1.367417 \\
\hline $23 . \mathrm{H}$ & -1.924125 & 2.150122 & -2.097262 \\
\hline $24 . \mathrm{H}$ & -0.938066 & 0.666086 & -2.102090 \\
\hline $25 . C$ & -3.641636 & -1.086851 & 1.064278 \\
\hline 26.0 & -0.199832 & 0.479260 & 1.183676 \\
\hline $27 . \mathrm{H}$ & 4.170620 & 2.020246 & -1.179188 \\
\hline $28 . \mathrm{C}$ & 3.141125 & 0.218752 & -0.593189 \\
\hline $29 . \mathrm{H}$ & 2.926420 & -0.773278 & -1.019790 \\
\hline $30 . \mathrm{C}$ & 3.822395 & 0.022663 & 0.774616 \\
\hline $31 . \mathrm{H}$ & 3.973768 & 0.999881 & 1.258681 \\
\hline 32. C & 3.077076 & -0.913090 & 1.752783 \\
\hline 33.0 & 2.123879 & -0.239197 & 2.596680 \\
\hline $34 . \mathrm{H}$ & 2.600481 & -1.733398 & 1.199586 \\
\hline $35 . \mathrm{H}$ & 3.835307 & -1.340088 & 2.416341 \\
\hline $36 . \mathrm{H}$ & 7.118069 & 0.758596 & -2.558196 \\
\hline $37 . \mathrm{C}$ & 8.086549 & -0.328213 & 0.764849 \\
\hline 38.0 & 7.220556 & -0.486597 & -0.395782 \\
\hline $39 . \mathrm{H}$ & 8.278067 & 0.736637 & 0.947370 \\
\hline $40 . \mathrm{H}$ & 7.631848 & -0.783739 & 1.650828 \\
\hline 41.0 & 5.103843 & -0.618543 & 0.530499 \\
\hline 42.0 & 3.351905 & 1.179001 & -2.828361 \\
\hline $43 . \mathrm{C}$ & 4.037013 & 1.002952 & -1.566609 \\
\hline $44 . \mathrm{H}$ & 3.290451 & 0.307801 & -3.266638 \\
\hline $45 . C$ & 5.995942 & 0.180309 & -0.285132 \\
\hline $46 . \mathrm{H}$ & 9.019783 & -0.837828 & 0.520307 \\
\hline $47 . \mathrm{C}$ & 5.406932 & 0.343599 & -1.697782 \\
\hline 48.0 & 6.241201 & 1.184651 & -2.516514 \\
\hline $49 . \mathrm{H}$ & 5.306595 & -0.657715 & -2.144900 \\
\hline $50 . \mathrm{H}$ & 6.126134 & 1.168459 & 0.190526 \\
\hline 51.0 & 1.901072 & 0.957952 & -0.443761 \\
\hline $52 . \mathrm{H}$ & 1.662631 & 1.255915 & -1.344380 \\
\hline $53 . \mathrm{H}$ & 1.255047 & -0.169190 & 2.137671 \\
\hline
\end{tabular}




\section{glc-glc}

glc-glc, bond energy: $-6858.65 \mathrm{kcal} / \mathrm{mol}$

$\begin{array}{rrrr}\text { 1.H } & -2.694748 & -2.681878 & 1.996749 \\ 2 . \mathrm{O} & 0.181719 & 1.599032 & -0.850018 \\ 3 . \mathrm{H} & -6.047719 & 2.170269 & -0.971447 \\ 4 . \mathrm{C} & -0.883888 & 0.740587 & -1.341678 \\ 5 . \mathrm{H} & -1.537590 & -1.492917 & 0.172757 \\ 6 . \mathrm{O} & -5.492105 & 0.142480 & -0.987040 \\ 7 . \mathrm{C} & -1.970189 & 0.586706 & -0.270538 \\ 8 . \mathrm{O} & -5.414528 & -0.930746 & 1.637198 \\ 9 . \mathrm{H} & -4.426202 & 1.246827 & 0.398917 \\ 10 . \mathrm{H} & -4.201415 & -1.807033 & 0.169046 \\ 11 . \mathrm{H} & -5.199904 & -1.555593 & 2.356782 \\ 12 . \mathrm{C} & -5.877831 & 1.358266 & -1.689807 \\ 13 . \mathrm{O} & -3.191175 & 0.328508 & -1.009462 \\ 14 . \mathrm{C} & -2.973200 & -0.758081 & 1.618282 \\ 15 . \mathrm{H} & -2.059787 & 1.533760 & 0.285203 \\ 16 . \mathrm{H} & 0.222431 & -0.428491 & 1.175256 \\ 17 . \mathrm{H} & -3.072341 & 0.118744 & 2.270365 \\ 18 . \mathrm{C} & -1.728268 & -0.568541 & 0.739989 \\ 19 . \mathrm{C} & -4.370699 & 0.300550 & -0.167807 \\ 20 . \mathrm{H} & -5.105588 & 1.653075 & -2.408213 \\ 21 . \mathrm{O} & -2.857542 & -1.877388 & 2.526691 \\ 22 . \mathrm{H} & -6.806293 & 1.125143 & -2.213610 \\ 23 . \mathrm{H} & -1.343254 & 1.200503 & -2.223681 \\ 24 . \mathrm{H} & -0.478577 & -0.240292 & -1.625680 \\ 25 . \mathrm{C} & -4.254894 & -0.892815 & 0.780345 \\ 26 . \mathrm{O} & -0.631796 & -0.290460 & 1.632728 \\ 27 . \mathrm{H} & 3.640681 & 1.997853 & -0.641972 \\ 28 . \mathrm{C} & 3.195996 & -0.014384 & 0.019659 \\ 29 . \mathrm{H} & 3.018801 & -0.997086 & -0.445214 \\ 30 . \mathrm{C} & 4.351078 & -0.154917 & 1.038019 \\ 31 . \mathrm{H} & 4.517807 & 0.820145 & 1.523505 \\ 32 . \mathrm{C} & 4.076151 & -1.206714 & 2.120776 \\ 33 . \mathrm{O} & 3.100485 & -0.749865 & 3.087253 \\ 34 . \mathrm{H} & 3.747590 & -2.145505 & 1.651247 \\ 35 . \mathrm{H} & 5.001769 & -1.395472 & 2.671177 \\ 36 . \mathrm{H} & 6.183271 & 1.434175 & -2.970329 \\ 38 & 8.358755 & 0.172784 & -0.372136 \\ \text { 39. } & 7.174254 & 0.008507 & -1.203751 \\ \text { 30 } & 8.477874 & 1.226901 & -0.092525 \\ \text { 3. } & & -0.446875 & 0.527807\end{array}$




$\begin{array}{lrrr}41 . \mathrm{O} & 5.547488 & -0.570020 & 0.340731 \\ 42 . \mathrm{O} & 2.559446 & 1.103246 & -2.109101 \\ 43 . \mathrm{C} & 3.580431 & 0.994748 & -1.078879 \\ 44 . \mathrm{H} & 2.523790 & 0.258920 & -2.599028 \\ 45 . \mathrm{C} & 5.991271 & 0.441487 & -0.595576 \\ 46 . \mathrm{H} & 9.204942 & -0.149948 & -0.980512 \\ 47 . \mathrm{C} & 4.934036 & 0.628528 & -1.696988 \\ 48 . \mathrm{O} & 5.313938 & 1.681693 & -2.601906 \\ 49 . \mathrm{H} & 4.842729 & -0.328064 & -2.234859 \\ 50 . \mathrm{H} & 6.150231 & 1.387308 & -0.048894 \\ 51 . \mathrm{O} & 2.018880 & 0.390743 & 0.757776 \\ 52 . \mathrm{H} & 1.369505 & 0.867259 & 0.170436 \\ 53 . \mathrm{H} & 2.366256 & -0.355878 & 2.573535 \\ 54 . \mathrm{H} & 0.905782 & 1.577871 & -1.523790\end{array}$




\section{A-T}

$\mathrm{A}-\mathrm{T}$, bond energy: $-4516.66 \mathrm{kcal} / \mathrm{mol}$

$\begin{array}{rrrr}\text { 1.N } & 0.953074 & -0.173131 & -0.001782 \\ \text { 2.C } & 1.564765 & -1.374502 & -0.000672 \\ \text { 3.N } & 2.882503 & -1.630488 & 0.000722 \\ \text { 4.C } & 3.611603 & -0.490825 & 0.000327 \\ \text { 5.C } & 3.107869 & 0.821593 & -0.000430 \\ \text { 6.C } & 1.699547 & 0.968609 & 0.000111 \\ 7 . \mathrm{N} & 4.983389 & -0.345077 & 0.000527 \\ \text { 8.C } & 5.250532 & 1.007233 & -0.001243 \\ 9 . \mathrm{N} & 4.150404 & 1.749330 & -0.001528 \\ 10 . \mathrm{N} & 1.068048 & 2.156517 & 0.001525 \\ 11 . \mathrm{H} & 0.896100 & -2.232059 & -0.001104 \\ 12 . \mathrm{H} & 5.669077 & -1.091957 & 0.000568 \\ 13 . \mathrm{H} & 6.263281 & 1.383489 & -0.002692 \\ 14 . \mathrm{H} & 1.606540 & 3.012183 & 0.002432 \\ 15 . \mathrm{H} & 0.041802 & 2.196175 & 0.000416 \\ 16 . \mathrm{N} & -1.886937 & -0.124556 & 0.001772 \\ 17 . \mathrm{C} & -2.501750 & -1.358569 & 0.000858 \\ 18 . \mathrm{N} & -3.883540 & -1.299298 & 0.001245 \\ 19 . \mathrm{C} & -4.590540 & -0.119273 & -0.000437 \\ 20 . \mathrm{C} & -3.976279 & 1.095569 & 0.000128 \\ 21 . \mathrm{C} & -2.521902 & 1.116118 & 0.001880 \\ 22 . \mathrm{O} & -1.834684 & 2.166124 & 0.002302 \\ 23 . \mathrm{O} & -1.872956 & -2.430038 & 0.000015 \\ 24 . \mathrm{C} & -4.730193 & 2.399465 & -0.000827 \\ 25 . \mathrm{H} & -0.832719 & -0.134286 & 0.002401 \\ 26 . \mathrm{H} & -4.376571 & -2.186195 & -0.000563 \\ 27 . \mathrm{H} & -5.668913 & -0.228913 & -0.002827 \\ 28 . \mathrm{H} & -4.473985 & 2.999888 & 0.880865 \\ 29 . \mathrm{H} & -5.809605 & 2.221945 & -0.001526 \\ 30 . \mathrm{H} & -4.472756 & 2.999403 & -0.882462\end{array}$

\section{G-C}

G-C, bond energy: $-4428.07 \mathrm{kcal} / \mathrm{mol}$

$\begin{array}{rrrr}1 . \mathrm{N} & -3.138905 & -0.533417 & 0.301547 \\ 2.0 & 0.787734 & 1.926324 & 0.293581 \\ 3 . \mathrm{N} & -1.217080 & 0.776625 & 0.296857 \\ 4 . \mathrm{C} & -2.596844 & 0.700532 & 0.299541 \\ 5 . \mathrm{N} & -3.388049 & 1.781137 & 0.300913\end{array}$




$\begin{array}{rrrr}\text { 6.C } & -2.697272 & 2.943164 & 0.300383 \\ 7 . \mathrm{C} & -1.303262 & 3.122259 & 0.298829 \\ \text { 8.C } & -0.470731 & 1.966679 & 0.296040 \\ 9 . \mathrm{N} & -3.218551 & 4.217641 & 0.302738 \\ 10 . \mathrm{C} & -2.152640 & 5.100847 & 0.302832 \\ 11 . \mathrm{N} & -0.983022 & 4.483219 & 0.300384 \\ 12 . \mathrm{H} & -2.565481 & -1.390165 & 0.296459 \\ 13 . \mathrm{H} & -4.147561 & -0.606763 & 0.300839 \\ 14 . \mathrm{H} & -0.670332 & -0.115516 & 0.294715 \\ 15 . \mathrm{H} & -4.202521 & 4.462177 & 0.303016 \\ 16 . \mathrm{H} & -2.304616 & 6.170343 & 0.302962 \\ 17 . \mathrm{O} & -1.631742 & -2.939090 & 0.291093 \\ 18 . \mathrm{N} & 2.286200 & -0.508448 & 0.295328 \\ 19 . \mathrm{N} & 0.309433 & -1.697873 & 0.292016 \\ 20 . \mathrm{C} & 2.421262 & -2.917040 & 0.302948 \\ 21 . \mathrm{C} & -0.373894 & -2.872597 & 0.294217 \\ 22 . \mathrm{N} & 0.359215 & -4.063542 & 0.300143 \\ 23 . \mathrm{C} & 1.723791 & -4.085148 & 0.304152 \\ 24 . \mathrm{C} & 1.663773 & -1.697264 & 0.296687 \\ 25 . \mathrm{H} & 3.504588 & -2.902767 & 0.307497 \\ 26 . \mathrm{H} & -0.167683 & -4.931232 & 0.301200 \\ 27 . \mathrm{H} & 2.189401 & -5.062552 & 0.309840 \\ 28 . \mathrm{H} & 1.746423 & 0.369027 & 0.293555 \\ 29 . \mathrm{H} & 3.296705 & -0.469697 & 0.297951\end{array}$

\section{References}

1. Stevens, K. \& Madder, A. Furan-modified oligonucleotides for fast, high-yielding and site-selective DNA inter-strand cross-linking with non-modified complements. Nucleic Acids Res 37, 1555-1565 (2009).

2. Schlegel, M.K. \& Meggers, E. Improved phosphoramidite building blocks for the synthesis of the simplified nucleic acid GNA. J Org Chem 74, 4615-4618 (2009).

3. te Velde, G., Bickelhaupt, F. M., Baerends, E. J., Fonseca Guerra, C., van Gisbergen, S. J. A., Snijders, J. G. \& Ziegler, T. Chemistry with ADF. J. Comput. Chem. 22, 931-967 (2001).

4. Fonseca Guerra, C., Snijders, J.G., te Velde, G. \& Baerends, E.J. Towards an order-N DFT method. Theor. Chem. Acc. 99, 391-403 (1998).

5. Computer code ADF, Version 2013.01: E. J. Baerends, J. Autschbach, A. Bérces, J. A. Berger, F. M. Bickelhaupt, C. Bo, P. L. de Boeij, P. M. Boerrigter, L. Cavallo, D. P. Chong, L. Deng, R. M. Dickson, D. E. Ellis, M. van Faassen, L. Fan, T. H. Fischer, C. Fonseca Guerra, S. J. A. van Gisbergen, J. A. Groeneveld, O. V. Gritsenko, M. Grüning, F. E. Harris, P. van den Hoek, C. R. Jacob, H. Jacobsen, L. Jensen, E. S. Kadantsev, G. van Kessel, R. Klooster, F. Kootstra, E. van Lenthe, D. A. McCormack, A. Michalak, J. Neugebauer, V. P. Nicu, V. P. Osinga, S. Patchkovskii, P. H. T. Philipsen, D. Post, C. C. 
Pye, W. Ravenek, P. Romaniello, P. Ros, P. R. T. Schipper, G. Schreckenbach, J. Snijders, M. Solà, M. Swart, D. Swerhone, G. te Velde, P. Vernooijs, L. Versluis, L. Visscher, O. Visser, F. Wang, T. A. Wesolowski, E. M. van Wezenbeek, G. Wiesenekker, S. K. Wolff, T. K. Woo, A. L. Yakovlev, T. Ziegler, Theoretical Chemistry, (SCM, Vrije Universiteit: Amsterdam, The Netherlands,).

6. Fonseca Guerra, C., van der Wijst, T., Poater, J., Swart, M. \& Bickelhaupt, F.M. Adenine versus guanine quartets in aqueous solution: dispersion-corrected DFT study on the differences in $\pi$-stacking and hydrogen-bonding behavior. Theor. Chem. Acc. 125, 245252 (2010).

7. Klamt, A. \& Schuurmann, G. COSMO: a new approach to dielectric screening in solvents with explicit expressions for the screening energy and its gradient. J. Chem. Soc., Perkin Trans. 2, 799-805 (1993).

8. Klamt, A. Conductor-like Screening Model for Real Solvents: A New Approach to the Quantitative Calculation of Solvation Phenomena. J Phys Chem 99, 2224-2235 (1995).

9. Pye, C.C. \& Ziegler, T. An implementation of the conductor-like screening model of solvation within the Amsterdam density functional package. Theor. Chem. Acc. 101, 396-408 (1999).

10. Fygenson, D.K. \& Goodman, M.F. Appendix. Gel kinetic analysis of polymerase fidelity in the presence of multiple enzyme DNA encounters. J Biol Chem 272, 27931-27935 (1997).

11. Moran, S., Ren, R.X. \& Kool, E.T. A thymidine triphosphate shape analog lacking Watson-Crick pairing ability is replicated with high sequence selectivity. Proc Natl Acad Sci U S A 94, 10506-10511 (1997). 